\title{
Angewandte
}

Supporting Information

(c) Wiley-VCH 2006

69451 Weinheim, Germany 


\title{
A Chiral Primary Amine-Thiourea Catalyst for the Highly Enantioselective Direct Conjugate Addition of $\alpha, \alpha$-Disubstituted Aldehydes to Nitroalkenes
}

\author{
Mathieu P. Lalonde, Yonggang Chen, and Eric N. Jacobsen
}

General Information: All reactions were performed under a nitrogen atmosphere in oven-dried round-bottomed flasks fitted with rubber septa or yellow polyethylene stoppers. Liquid reagents were transferred with stainless steel syringes. Flash Chromatography was performed with EM Science silica gel 60 (230-400 mesh).

Materials: Dichloromethane was distilled from $\mathrm{CaH}_{2}$ at 760 Torr. Commercially available aldehydes were purified by distillation from anhydrous calcium sulfate and/or column chromatography prior to use. Commercially available nitroalkenes were used as received. trans- $\beta$-nitrostyrene, trans-4-methoxy- $\beta$-nitrostyrene, trans-2-(2nitrovinyl)thiophene, trans-2-(2-nitrovinyl)furan, trans-4-bromo- $\beta$-nitrostyrene, trans- $\beta$ nitro-2-(trifluoromethyl)styrene, 2-phenylpropionaldehyde, 2-methylpentanal, 2,6dimethyl-5-heptenal, 2-methyl-3-(3,4-methylenedioxyphenyl)propanal were purchased from Aldrich. 3-(2-nitroethenyl)pyridine was purchased from TCI America. 4-fluoro- $\beta$ nitrostyrene was purchased from Fluka. (R)-3,3-dimethyl-2-butylamine was purchased from Lancaster. (E)-1-nitroprop-1-ene, ${ }^{[1]}(E)$-1-nitrohex-1-ene ${ }^{[1]}(E)$-3-(benzyloxy)-1nitroprop-1-ene, ${ }^{[1]}$ trans-3,3,3-trifluoro-1-nitroprop-1-ene, ${ }^{[2]}$ 3-(tertbutyldimethylsilyloxy)-2-methylpropanal ${ }^{[3]} 2$-(4-methoxybenzyloxy)propanal ${ }^{[4]}$ thiourea catalysts ${ }^{[5]}$ 1-2 were prepared according to previously published procedures.

Instrumentation: Proton nuclear magnetic resonance $\left({ }^{1} \mathrm{H}\right.$ NMR $)$ spectra and carbon nuclear magnetic resonance $\left({ }^{13} \mathrm{C}\right.$ NMR) spectra were recorded on a Varian Mercury-400 $(400 \mathrm{MHz})$ NMR spectrometer. Chemical shifts for protons are reported in parts per million downfield from tetramethylsilane and are referenced to the solvent residual peak $\left(\mathrm{CHCl}_{3}: \delta 7.26\right)$. Chemical shifts for carbon are reported in parts per million downfield from tetramethylsilane and are referenced to the carbon resonances of the solvent $\left(\mathrm{CDCl}_{3}: \delta 77.0\right)$. Data are represented as follows: chemical shift, integration, multiplicity ( $\mathrm{br}=$ broad, $\mathrm{s}=$ singlet, $\mathrm{d}=$ doublet, $\mathrm{t}=$ triplet, $\mathrm{q}=$ quartet, $\mathrm{m}=$ multiplet $)$, and coupling constants in Hertz (Hz). Infrared (IR) spectra were obtained using a Mattson Galaxy Series FTIR 3000 spectrophotometer referenced to a polystyrene standard. Data are represented as follows: frequency of absorption $\left(\mathrm{cm}^{-1}\right)$, intensity of absorption $(\mathrm{s}=$ strong, $\mathrm{m}=$ medium, $\mathrm{w}=$ weak). Optical rotations were measured using a $2.0 \mathrm{~mL}$ cell

[1] D. Lucet, S. Sabelle, O. Kostelitz, T. Le Gall, C. Mioskowski, Eur. J. Org. Chem. 1999, 2583-2591.

[2] M. Molteni, A. Volonterio, M. Zanda, Org. Lett. 2003, 5, 3887-3890.

[3] S.-I. Kiyooka, K. A. Shahid, F. Goto, M. Okazaki, Y. Shuto, J. Org. Chem. 2003, 68, 7967-7978.

[4] W. Yu, Y. Zhang, Z. Jin, Org. Lett. 2001, 3, 1447-1450. Procedure was followed using racemic ethyl lactate.

[5] A. G. Wenzel, E. N. Jacobsen, J. Am. Chem. Soc. 2002, 124, 12964-12965. 
with a $1.0 \mathrm{dm}$ path length on a Jasco DIP 370 digital polarimeter. Melting points were measured on a Mel-Temp apparatus, and are uncorrected. The mass spectroscopic data were obtained at the Harvard University mass spectrometry facility. Chiral HPLC analysis was performed on a Hewlett Packard 1050 Series instrument. Chiral SFC analysis was performed on a Berger instrument.

General Procedure for the Addition of $\alpha, \alpha$-Disubstituted Aldehydes to Nitroalkenes.
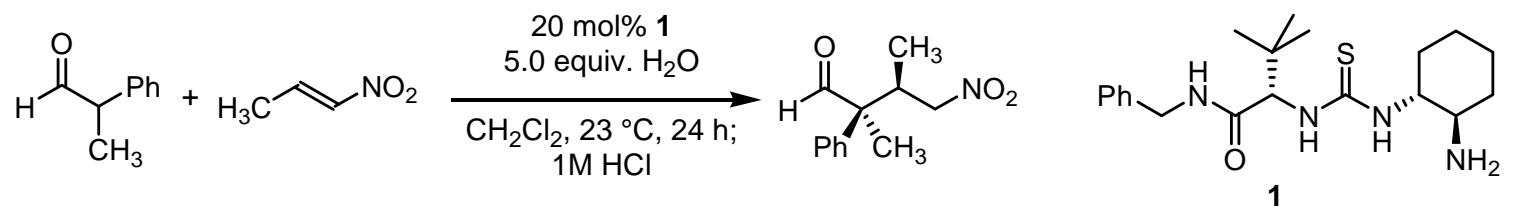

(2S,3R)-2,3-dimethyl-4-nitro-2-phenylbutanal (6): Under a positive pressure of nitrogen at room temperature, thiourea catalyst $1(75.3 \mathrm{mg}, 0.20 \mathrm{mmol}, 20 \mathrm{~mol} \%)$ was loaded into an oven-dried $25 \mathrm{~mL}$ round-bottomed flask, equipped with a magnetic stir bar, rubber septum, and nitrogen inlet. The catalyst was dissolved in dichloromethane $(6.7 \mathrm{~mL})$. Water $(90.1 \mu \mathrm{L}, 5.0 \mathrm{mmol}, 5.0$ equiv.) and 2-phenylpropionaldehyde (265.4 $\mu \mathrm{L}, 2.0 \mathrm{mmol}, 2.0$ equiv.) were subsequently added via syringe. The resulting clear colorless solution was stirred for approximately two minutes. 1-nitropropene $(87.1 \mathrm{mg}$, $1.0 \mathrm{mmol}, 1.0$ equiv.) was added via syringe resulting in a light yellow solution. The rubber septum was quickly replaced with a yellow polyethylene stopper (to prevent dichloromethane evaporation) and the reaction mixture was stirred for 24 hours at room temperature. Aqueous hydrochloric acid solution $(1 \mathrm{M}, 7 \mathrm{~mL})$ was added to the reaction flask and the resulting biphasic mixture was stirred vigorously for 5 minutes at room temperature. The biphasic mixture was transferred to a separatory funnel and additional portions of dichloromethane $(30 \mathrm{~mL})$ and $1 \mathrm{M} \mathrm{HCl}(30 \mathrm{~mL})$ were added. The phases were separated and the aqueous layer was washed with dichloromethane $(30 \mathrm{~mL})$. The organic layers were combined and washed with saturated aqueous sodium bicarbonate solution $(30 \mathrm{~mL})$, saturated aqueous sodium chloride solution $(30 \mathrm{~mL})$, dried over anhydrous sodium sulfate, filtered, and concentrated in vacuo. The resulting yellow residue was purified by chromatography on silica ( $8 \%$ diethyl ether/hexanes), providing the title compound as a colorless to light yellow liquid in $91 \%$ yield $(201.1 \mathrm{mg}$ ) with a $23: 1$ diastereomeric ratio and 99\% enantiomeric excess (major diastereomer) as determined by HPLC (Chiralpak AD-H, 2.0\% isopropanol/Hexanes, $1.0 \mathrm{~mL} / \mathrm{min}, 230 \mathrm{~nm} ; t_{r}($ minor enantiomer, minor diastereomer $)=11.83 \mathrm{~min}, t_{r}$ (major enantiomer, minor diastereomer) $=12.87 \mathrm{~min}, t_{r}($ minor enantiomer, major diastereomer $)=13.82 \mathrm{~min}, t_{r}$ (major enantiomer, major diastereomer $)=15.48 \mathrm{~min}) \cdot[\alpha]^{25}=+88.6(\mathrm{c}=0.0200 \mathrm{~g} / 2.0 \mathrm{~mL}$, chloroform $){ }^{1} \mathrm{H}$ NMR (400 MHz, CDCl $): \delta 9.47(1 \mathrm{H}, \mathrm{s}), 7.42(2 \mathrm{H}, \mathrm{t}, \mathrm{J}=7.3 \mathrm{~Hz}), 7.34(1 \mathrm{H}, \mathrm{t}, \mathrm{J}=7.3$ $\mathrm{Hz}), 7.24(2 \mathrm{H}, \mathrm{d}, \mathrm{J}=7.3 \mathrm{~Hz}), 4.57(1 \mathrm{H}, \mathrm{dd}, \mathrm{J}=3.3,12.0 \mathrm{~Hz}), 4.19(1 \mathrm{H}, \mathrm{dd}, \mathrm{J}=10.6,12.0$ $\mathrm{Hz}), 3.17(1 \mathrm{H}, \mathrm{m}), 1.48(3 \mathrm{H}, \mathrm{s}), 0.81(3 \mathrm{H}, \mathrm{d}, \mathrm{J}=7.0 \mathrm{~Hz}) \cdot{ }^{13} \mathrm{C}\left(100 \mathrm{MHz}, \mathrm{CDCl}_{3}\right): \delta 200.6$, 137.4, 129.4, 128.2, 127.4, 78.8, 55.9, 37.1, 14.6, 13.2. IR (neat): 3060 (w), $2981(\mathrm{~m})$, $2819(\mathrm{w}), 2719$ (w), 1722 (s), 1533 (s), $1496(\mathrm{~m}), 1446$ (m), 1377 (s), $763(\mathrm{~m}), 702(\mathrm{~m})$. HRMS (ESI): expected for $\left[\mathrm{C}_{12} \mathrm{H}_{15} \mathrm{NO}_{3}+\mathrm{NH}_{4}\right]^{+}: 239.1396$, found: 239.1402 . 


\section{Racemic 2,3-dimethyl-4-nitro-2-phenylbutanal}

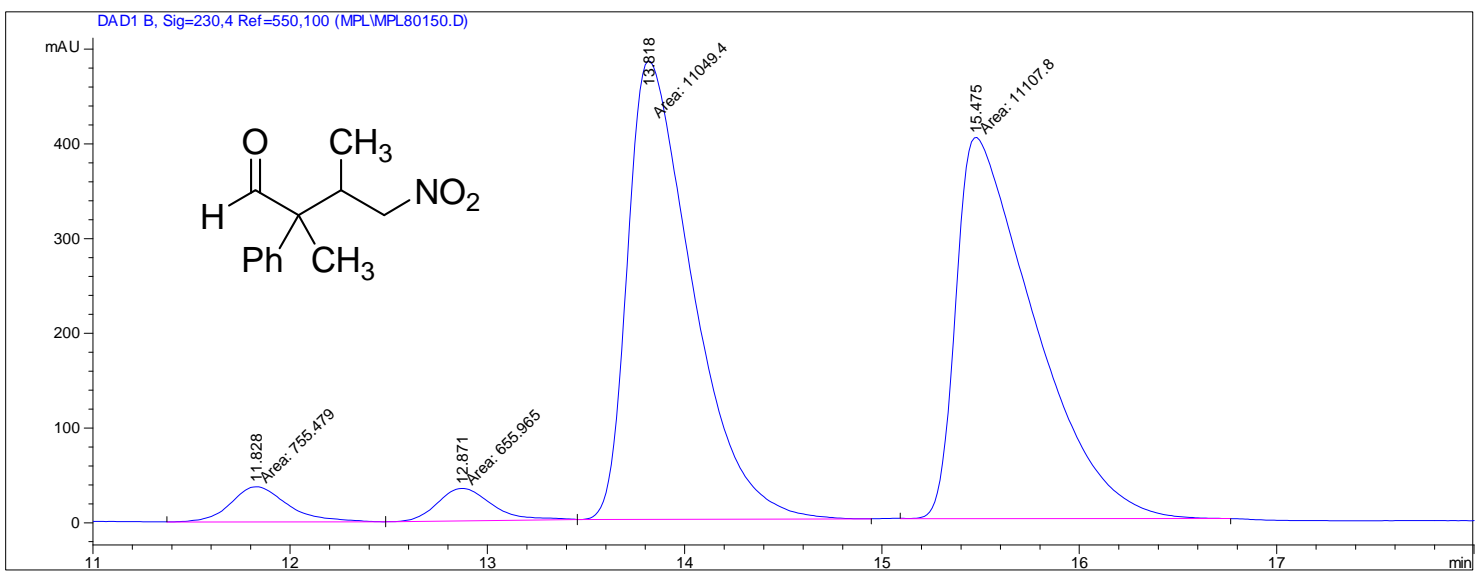

\begin{tabular}{|l|l|l|l|l|l|l|}
\hline $\begin{array}{l}\text { Peak } \\
\#\end{array}$ & $\begin{array}{l}\text { RetTime } \\
{[\mathrm{min}]}\end{array}$ & Type & $\begin{array}{l}\text { Width } \\
{[\mathrm{min}]}\end{array}$ & $\begin{array}{l}\text { Area } \\
{[\mathrm{mAU} * \mathrm{~s}]}\end{array}$ & $\begin{array}{l}\text { Height } \\
{[\mathrm{mAU}]}\end{array}$ & $\begin{array}{l}\text { Area } \\
{[\%]}\end{array}$ \\
\hline 1 & 11.828 & $\mathrm{MM}$ & 0.3336 & 744.17249 & 37.17384 & 3.1239 \\
\hline 2 & 12.871 & $\mathrm{MM}$ & 0.3334 & 700.51599 & 35.01959 & 2.9407 \\
\hline 3 & 13.818 & $\mathrm{MM}$ & 0.3825 & $1.11430 \mathrm{e} 4$ & 485.54669 & 46.7766 \\
\hline 4 & 15.475 & $\mathrm{MM}$ & 0.4643 & $1.12340 \mathrm{e} 4$ & 403.25229 & 47.1588 \\
\hline Totals: & & & & $2.38216 \mathrm{e} 4$ & 960.99240 & \\
\hline
\end{tabular}

\section{Enantioenriched (2S,3R)-2,3-dimethyl-4-nitro-2-phenylbutanal}

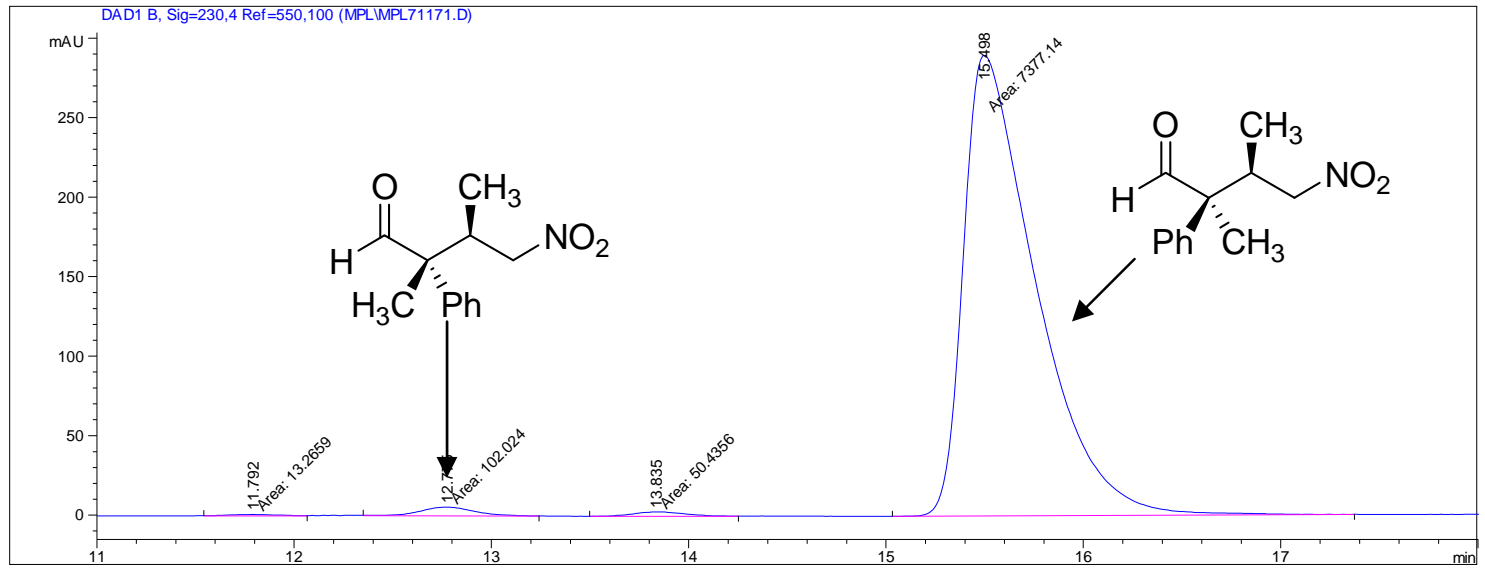

\begin{tabular}{|l|l|l|l|l|l|l|}
\hline $\begin{array}{l}\text { Peak } \\
\#\end{array}$ & $\begin{array}{l}\text { RetTime } \\
{[\mathrm{min}]}\end{array}$ & Type & $\begin{array}{l}\text { Width } \\
{[\mathrm{min}]}\end{array}$ & $\begin{array}{l}\text { Area } \\
{[\mathrm{mAU} * \mathrm{~s}]}\end{array}$ & $\begin{array}{l}\text { Height } \\
{[\mathrm{mAU}]}\end{array}$ & $\begin{array}{l}\text { Area } \\
{[\%]}\end{array}$ \\
\hline 1 & 11.792 & MM & 0.2958 & 14.55923 & $8.20360 \mathrm{e}-1$ & 0.1922 \\
\hline 2 & 12.775 & MM & 0.3166 & 105.39117 & 5.54822 & 1.3912 \\
\hline 3 & 13.835 & MM & 0.3109 & 50.89827 & 2.72861 & 0.6719 \\
\hline 4 & 15.498 & MM & 0.4254 & 7404.60938 & 290.08578 & 97.7447 \\
\hline Totals: & & & & 7575.45805 & 299.18297 & \\
\hline
\end{tabular}


(2S,3R)-2-methyl-3-(nitromethyl)-2-phenylheptanal (5): General procedure, chromatography on silica (5\% diethyl ether/hexanes), colorless to light yellow liquid, $54 \%$ yield (143.8 mg), 28:1 dr as determined by HPLC (Econosphere CN 5U + Chiralcel OD-H, $5.0 \%$ isopropanol/hexanes, $1.0 \mathrm{~mL} / \mathrm{min}, 208 \mathrm{~nm}$; $t_{r}$ (major enantiomer, minor diastereomer $)=13.80 \mathrm{~min}, t_{r}($ minor enantiomer, minor diastereomer $)=15.04, t_{r}($ minor enantiomer, major diastereomer) $=16.53 \mathrm{~min}, t_{r}$ (major enantiomer, major diastereomer) $=18.87$ ), $96 \%$ ee (major diastereomer) as determined by HPLC (Chiralcel OD-H, 5.0\% isopropanol/hexanes, $1.0 \mathrm{~mL} / \mathrm{min}, 208 \mathrm{~nm}$; $t_{r}$ (minor enantiomer, major diastereomer) $=$ $12.38 \mathrm{~min}, t_{r}($ major enantiomer, major diastereomer $\left.)=15.20 \mathrm{~min}\right) .[\alpha]^{25}=+59.8(\mathrm{c}=$ $0.0200 \mathrm{~g} / 2.0 \mathrm{~mL}$, chloroform). ${ }^{1} \mathrm{H} \mathrm{NMR}\left(400 \mathrm{MHz}, \mathrm{CDCl}_{3}\right): \delta 9.49(1 \mathrm{H}, \mathrm{s}), 7.41(2 \mathrm{H}, \mathrm{t}, \mathrm{J}$ $=7.3 \mathrm{~Hz}), 7.33(1 \mathrm{H}, \mathrm{t}, \mathrm{J}=7.3 \mathrm{~Hz}), 7.29(2 \mathrm{H}, \mathrm{d}, \mathrm{J}=7.3 \mathrm{~Hz}), 4.48(1 \mathrm{H}, \mathrm{dd}, \mathrm{J}=4.2,13.5$ $\mathrm{Hz}), 4.28(1 \mathrm{H}, \mathrm{dd}, \mathrm{J}=7.3,13.5 \mathrm{~Hz}), 3.12(1 \mathrm{H}, \mathrm{m}), 1.47(3 \mathrm{H}, \mathrm{s}), 1.47-0.98(6 \mathrm{H}, \mathrm{m}), 0.72$ $(3 \mathrm{H}, \mathrm{t}, \mathrm{J}=7.0 \mathrm{~Hz}) .{ }^{13} \mathrm{C}\left(100 \mathrm{MHz}, \mathrm{CDCl}_{3}\right): \delta 200.9,137.6,129.3,128.1,127.5,77.7$, 57.0, 41.9, 29.9, 29.2, 22.6, 15.2, 13.8. IR (neat): 2958 (m), 2933 (m), $2864(\mathrm{~m}), 2716$ (w), $1722(\mathrm{~s}), 1553$ (s), 1447 (m), 1380 (m), 762(m), 702 (m). HRMS (ESI): expected for $\left[\mathrm{C}_{15} \mathrm{H}_{21} \mathrm{NO}_{3}+\mathrm{NH}_{4}\right]^{+}: 281.1865$, found: 281.1877 .

\section{Racemic 2-methyl-3-(nitromethyl)-2-phenylheptanal}

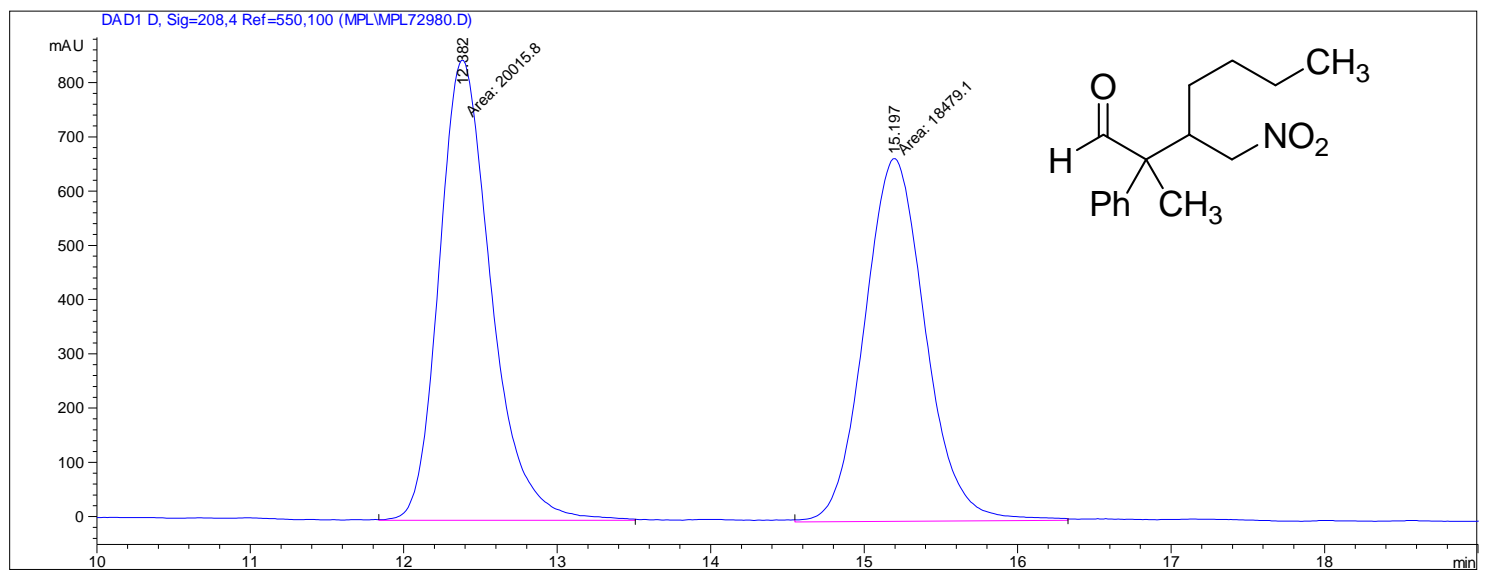

\begin{tabular}{|l|l|l|l|l|l|l|}
\hline $\begin{array}{l}\text { Peak } \\
\#\end{array}$ & $\begin{array}{l}\text { RetTime } \\
{[\mathrm{min}]}\end{array}$ & Type & $\begin{array}{l}\text { Width } \\
{[\mathrm{min}]}\end{array}$ & $\begin{array}{l}\text { Area } \\
{[\mathrm{mAU} * \mathrm{~s}]}\end{array}$ & $\begin{array}{l}\text { Height } \\
{[\mathrm{mAU}]}\end{array}$ & $\begin{array}{l}\text { Area } \\
{[\%]}\end{array}$ \\
\hline 1 & 12.382 & $\mathrm{MM}$ & 0.3899 & $1.98279 \mathrm{e} 4$ & 847.53461 & 51.8699 \\
\hline 2 & 15.197 & $\mathrm{MM}$ & 0.4595 & $1.83983 \mathrm{e} 4$ & 667.27185 & 48.1301 \\
\hline Totals: & & & & $3.82262 \mathrm{e} 4$ & 1514.80646 & \\
\hline
\end{tabular}




\section{Enantioenriched (2S,3R)-2-methyl-3-(nitromethyl)-2-phenylheptanal}

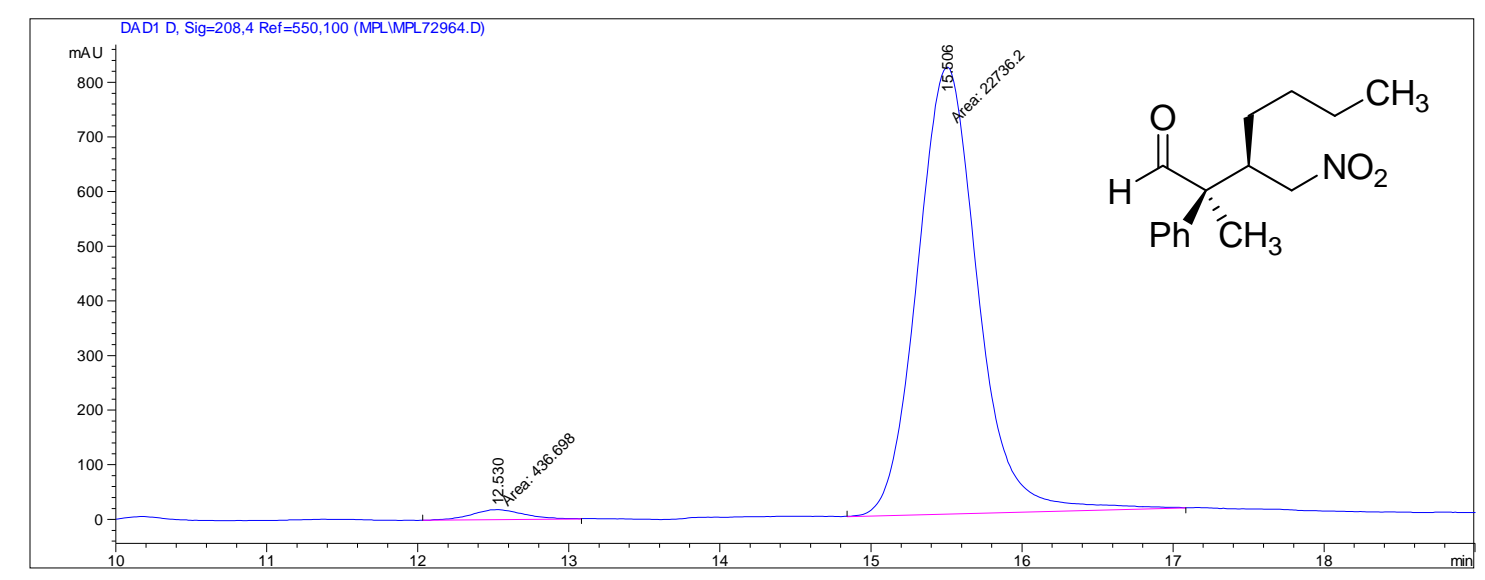

\begin{tabular}{|l|l|l|l|l|l|l|}
\hline $\begin{array}{l}\text { Peak } \\
\#\end{array}$ & $\begin{array}{l}\text { RetTime } \\
{[\mathrm{min}]}\end{array}$ & Type & $\begin{array}{l}\text { Width } \\
{[\mathrm{min}]}\end{array}$ & $\begin{array}{l}\text { Area } \\
{\left[\mathrm{mAU}^{*} \mathrm{~s}\right]}\end{array}$ & $\begin{array}{l}\text { Height } \\
{[\mathrm{mAU}]}\end{array}$ & $\begin{array}{l}\text { Area } \\
{[\%]}\end{array}$ \\
\hline 1 & 12.530 & $\mathrm{MM}$ & 0.4030 & 448.04437 & 18.52734 & 1.9355 \\
\hline 2 & 15.506 & $\mathrm{MM}$ & 0.4625 & $2.27005 \mathrm{e} 4$ & 818.05493 & 98.0645 \\
\hline Totals: & & & & $2.31485 \mathrm{e} 4$ & 836.58227 & \\
\hline
\end{tabular}

(2S,3R)-4,4,4-trifluoro-2-methyl-3-(nitromethyl)-2-phenylbutanal (7): General procedure, chromatography on silica (10\% diethyl ether/hexanes), colorless to light yellow liquid, $34 \%$ yield $(92.6 \mathrm{mg}),>50: 1 \mathrm{dr}$ (the presence of another diastereomer could not be detected by either ${ }^{1} \mathrm{H}$ NMR, SFC, or HPLC), $97 \%$ ee (major diastereomer) as determined by HPLC (Chiralpak AD-H, 2.0\% isopropanol/hexanes, $1.0 \mathrm{~mL} / \mathrm{min}, 208 \mathrm{~nm}$; $t_{r}($ minor enantiomer, major diastereomer $)=11.36 \mathrm{~min}, t_{r}$ (major enantiomer, major diastereomer $)=12.40 \mathrm{~min}) .[\alpha]^{25}=+140.1(\mathrm{c}=0.0210 \mathrm{~g} / 2.0 \mathrm{~mL}$, chloroform $){ }^{1} \mathrm{H}$ NMR $\left(400 \mathrm{MHz}, \mathrm{CDCl}_{3}\right): \delta 9.28(1 \mathrm{H}, \mathrm{s}), 7.45(2 \mathrm{H}, \mathrm{t}, \mathrm{J}=7.3 \mathrm{~Hz}), 7.38(1 \mathrm{H}, \mathrm{t}, \mathrm{J}=7.3 \mathrm{~Hz}), 7.28$ $(2 \mathrm{H}, \mathrm{t}, \mathrm{J}=7.3 \mathrm{~Hz}), 4.72(1 \mathrm{H}, \mathrm{dd}, \mathrm{J}=8.1,15.4 \mathrm{~Hz}), 4.48(1 \mathrm{H}, \mathrm{dd}, \mathrm{J}=2.2,15.4 \mathrm{~Hz}), 4.20$ $(1 \mathrm{H}, \mathrm{m}), 1.71(3 \mathrm{H}, \mathrm{s}) .{ }^{13} \mathrm{C}\left(100 \mathrm{MHz}, \mathrm{CDCl}_{3}\right): \delta 197.8,135.1,129.5,128.9,127.2,126.1$ $(1 \mathrm{C}, \mathrm{q}, \mathrm{J}=282.5 \mathrm{~Hz}), 124.7,71.5,46.7(1 \mathrm{C}, \mathrm{q}, \mathrm{J}=25.6 \mathrm{~Hz}), 16.1$. IR (neat): $3062(\mathrm{~m})$, $3032(\mathrm{~m}), 2981(\mathrm{~m}), 2828(\mathrm{~m}), 2722(\mathrm{~m}), 1721(\mathrm{~s}), 1567(\mathrm{~s}), 1497(\mathrm{~m}), 1448(\mathrm{~m}), 1380$ (s), $1249(\mathrm{~s}), 1178(\mathrm{~s}), 1100(\mathrm{~s}), 1001(\mathrm{~m}), 763(\mathrm{~m}), 701$ (s), $543(\mathrm{~m})$. LRMS (ESI): 296.3 $(100 \%)\left[\mathrm{C}_{12} \mathrm{H}_{12} \mathrm{~F}_{3} \mathrm{NO}_{3}+\mathrm{Na}\right]^{+}$. 


\section{Racemic 4,4,4-trifluoro-2-methyl-3-(nitromethyl)-2-phenylbutanal}

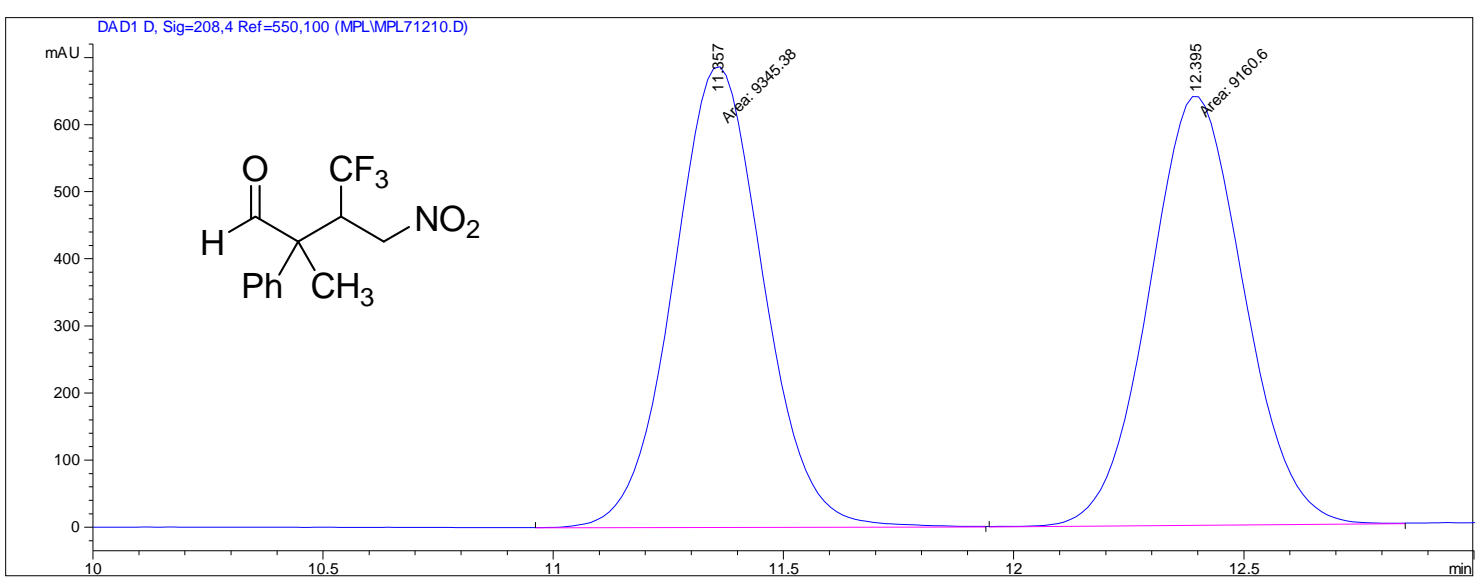

\begin{tabular}{|l|l|l|l|l|l|l|}
\hline $\begin{array}{l}\text { Peak } \\
\#\end{array}$ & $\begin{array}{l}\text { RetTime } \\
{[\mathrm{min}]}\end{array}$ & Type & $\begin{array}{l}\text { Width } \\
{[\mathrm{min}]}\end{array}$ & $\begin{array}{l}\text { Area } \\
{\left[\mathrm{mAU}^{*} \mathrm{~s}\right]}\end{array}$ & $\begin{array}{l}\text { Height } \\
{[\mathrm{mAU}]}\end{array}$ & $\begin{array}{l}\text { Area } \\
{[\%]}\end{array}$ \\
\hline 1 & 11.357 & $\mathrm{PV}$ & 0.2112 & 9325.99316 & 687.88574 & 50.1677 \\
\hline 2 & 12.395 & $\mathrm{MM}$ & 0.2403 & 9263.65332 & 642.42816 & 49.8323 \\
\hline Totals: & & & & $1.85896 \mathrm{e} 4$ & 1330.31390 & \\
\hline
\end{tabular}

\section{Enantioenriched (2S,3R)-4,4,4-trifluoro-2-methyl-3-(nitromethyl)-2-phenylbutanal}

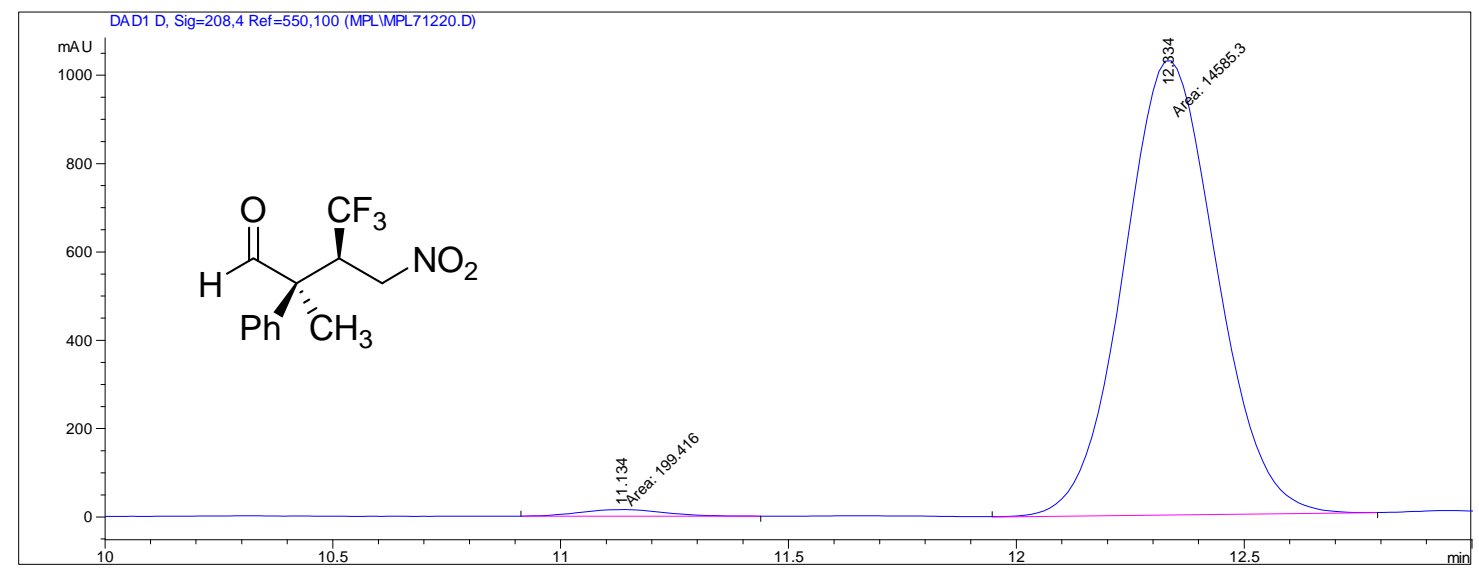

\begin{tabular}{|l|l|l|l|l|l|l|}
\hline $\begin{array}{l}\text { Peak } \\
\#\end{array}$ & $\begin{array}{l}\text { RetTime } \\
{[\mathrm{min}]}\end{array}$ & Type & $\begin{array}{l}\text { Width } \\
{[\mathrm{min}]}\end{array}$ & $\begin{array}{l}\text { Area } \\
{\left[\mathrm{mAU}^{*} \mathrm{~s}\right]}\end{array}$ & $\begin{array}{l}\text { Height } \\
{[\mathrm{mAU}]}\end{array}$ & $\begin{array}{l}\text { Area } \\
{[\%]}\end{array}$ \\
\hline 1 & 11.134 & $\mathrm{MM}$ & 0.2359 & 229.92702 & 16.24365 & 1.5519 \\
\hline 2 & 12.334 & $\mathrm{MM}$ & 0.2357 & $1.45856 \mathrm{e} 4$ & 1031.53821 & 98.4481 \\
\hline Totals: & & & & $1.48155 \mathrm{e} 4$ & 1047.78186 & \\
\hline
\end{tabular}



procedure, chromatography on silica (20\% diethyl ether/hexanes), colorless to light yellow liquid, $87 \%$ yield $(284.3 \mathrm{mg}),>50: 1 \mathrm{dr}$ (the presence of another diastereomer could not be detected by either ${ }^{1} \mathrm{H}$ NMR, SFC, or HPLC), 99\% ee (major diastereomer) as determined by HPLC (Chiralpak AD-H, 5\% isopropanol/hexanes, $1.0 \mathrm{~mL} / \mathrm{min}, 230$ $\mathrm{nm}$; $t_{r}$ (major enantiomer, major diastereomer $)=10.53 \mathrm{~min}, t_{r}($ minor enantiomer, major diastereomer $)=11.73 \mathrm{~min}) .[\alpha]^{25}=+92.2(\mathrm{c}=0.0240 \mathrm{~g} / 2.0 \mathrm{~mL}$, chloroform $) .{ }^{1} \mathrm{H}$ NMR $\left(400 \mathrm{MHz}, \mathrm{CDCl}_{3}\right): \delta 9.41(1 \mathrm{H}, \mathrm{s}), 7.43-7.21(10 \mathrm{H}, \mathrm{m}), 4.70(1 \mathrm{H}, \mathrm{dd}, \mathrm{J}=9.5,13.0 \mathrm{~Hz})$, $4.50(1 \mathrm{H}, \mathrm{dd}, \mathrm{J}=3.3,13.0 \mathrm{~Hz}), 4.36(1 \mathrm{H}, \mathrm{d}, \mathrm{J}=11.9 \mathrm{~Hz}), 4.27(1 \mathrm{H}, \mathrm{d}, \mathrm{J}=11.9 \mathrm{~Hz}), 3.34$ $(2 \mathrm{H}, \mathrm{m}), 3.17(1 \mathrm{H}, \mathrm{dd}, \mathrm{J}=4.0,9.5 \mathrm{~Hz}), 1.57(3 \mathrm{H}, \mathrm{s}) .{ }^{13} \mathrm{C}\left(100 \mathrm{MHz}, \mathrm{CDCl}_{3}\right): \delta 200.0$, $137.5,137.2,129.2,128.3,128.1,127.7,127.5,127.2,67.5,55.1,42.0,16.3$. IR (neat): $3062(\mathrm{~m}), 3030(\mathrm{~m}), 2863(\mathrm{~m}), 2814(\mathrm{~m}), 2717(\mathrm{~m}), 1722(\mathrm{~s}), 1555(\mathrm{~s}), 1496(\mathrm{~m}), 1454$ (m), $1379(\mathrm{~m}), 1104(\mathrm{~m}), 740(\mathrm{~m}), 700(\mathrm{~m})$. HRMS (ESI): expected for $\left[\mathrm{C}_{19} \mathrm{H}_{21} \mathrm{NO}_{4}+\mathrm{NH}_{4}-\right.$ ]$^{+}: 345.1814$, found: 345.1817 .

\section{Racemic 4-(benzyloxy)-2-methyl-3-(nitromethyl)-2-phenylbutanal}

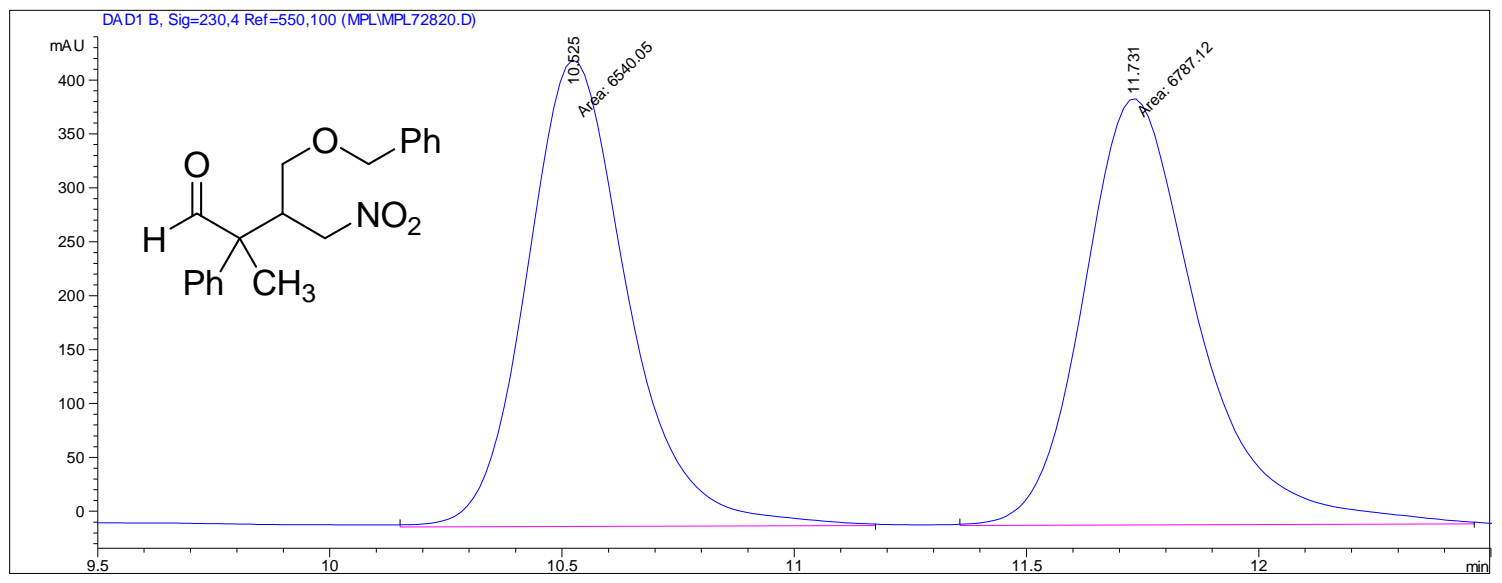

\begin{tabular}{|l|l|l|l|l|l|l|}
\hline $\begin{array}{l}\text { Peak } \\
\#\end{array}$ & $\begin{array}{l}\text { RetTime } \\
{[\mathrm{min}]}\end{array}$ & Type & $\begin{array}{l}\text { Width } \\
{[\mathrm{min}]}\end{array}$ & $\begin{array}{l}\text { Area } \\
{\left[\mathrm{mAU}^{*} \mathrm{~s}\right]}\end{array}$ & $\begin{array}{l}\text { Height } \\
{[\mathrm{mAU}]}\end{array}$ & $\begin{array}{l}\text { Area } \\
{[\%]}\end{array}$ \\
\hline 1 & 10.525 & $\mathrm{MM}$ & 0.2523 & 6561.72607 & 433.44318 & 48.9134 \\
\hline 2 & 11.731 & $\mathrm{MM}$ & 0.2881 & 6853.26660 & 396.42261 & 51.0866 \\
\hline Totals: & & & & $1.34150 \mathrm{e} 4$ & 829.86578 & \\
\hline
\end{tabular}


Enantioenriched (2S, 3R)-4-(benzyloxy)-2-methyl-3-(nitromethyl)-2-phenylbutanal

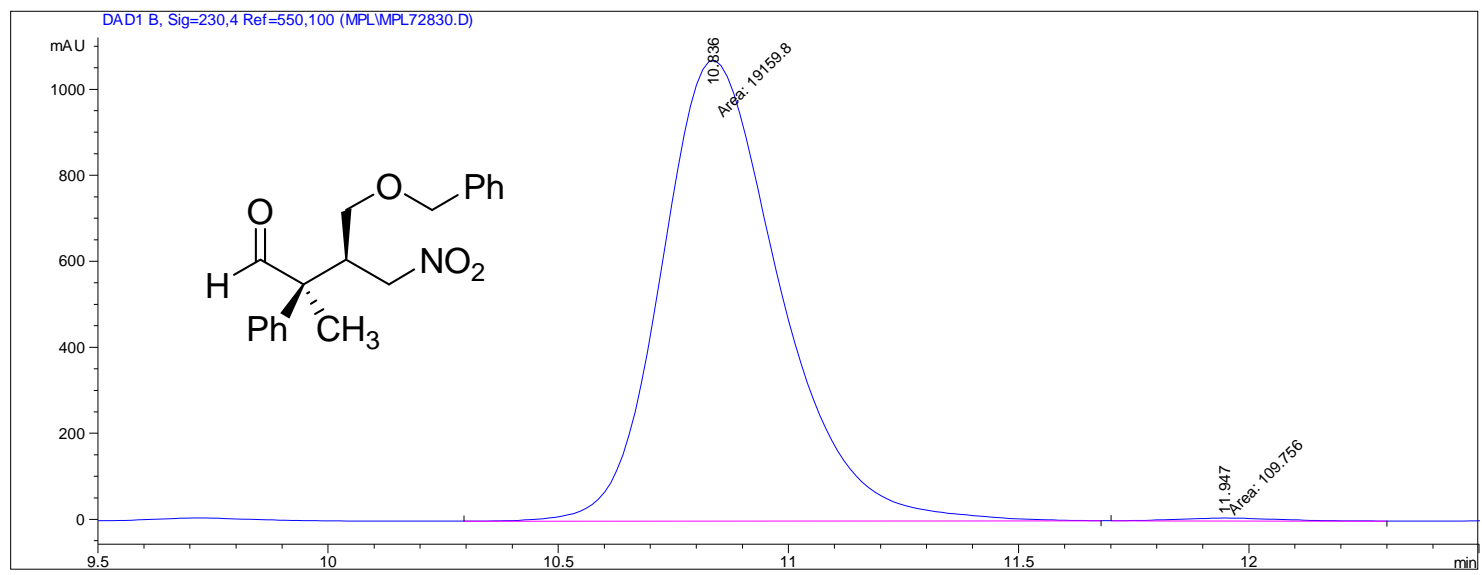

\begin{tabular}{|l|l|l|l|l|l|l|}
\hline $\begin{array}{l}\text { Peak } \\
\#\end{array}$ & $\begin{array}{l}\text { RetTime } \\
{[\mathrm{min}]}\end{array}$ & Type & $\begin{array}{l}\text { Width } \\
{[\mathrm{min}]}\end{array}$ & $\begin{array}{l}\text { Area } \\
{\left[\mathrm{mAU}^{*} \mathrm{~s}\right]}\end{array}$ & $\begin{array}{l}\text { Height } \\
{[\mathrm{mAU}]}\end{array}$ & $\begin{array}{l}\text { Area } \\
{[\%]}\end{array}$ \\
\hline 1 & 10.836 & $\mathrm{MM}$ & 0.2982 & $1.91983 \mathrm{e} 4$ & 1072.96790 & 99.3543 \\
\hline 2 & 11.947 & MM & 0.2888 & 124.76044 & 7.19885 & 0.6457 \\
\hline Totals: & & & & $1.93230 \mathrm{e} 4$ & 1080.16675 & \\
\hline
\end{tabular}

(R)-2,6-dimethyl-2-((R)-1-nitropropan-2-yl)hept-5-enal + (S)-2,6-dimethyl-2-((R)-1nitropropan-2-yl)hept-5-enal (9): General procedure, chromatography on silica (8\% diethyl ether/hexanes) diastereomers could not be separated, colorless to light yellow liquid, 61\% yield, 3.3:1 dr, 99\% ee (major diastereomer) as determined by SFC (Chiralcel OD-H, 2.0\% methanol/ $\mathrm{CO}_{2}, 2.0 \mathrm{~mL} / \mathrm{min}, 208 \mathrm{~nm}, 30^{\circ} \mathrm{C}$; $t_{r}$ (minor enantiomer, minor diastereomer $)=2.80 \mathrm{~min}, t_{r}($ minor enantiomer, major diastereomer $)=3.09 \mathrm{~min}$, $t_{r}$ (major enantiomer, major diastereomer $)=4.23 \mathrm{~min}, t_{r}$ (major enantiomer, minor diastereomer $)=4.66 \mathrm{~min}) .[\alpha]^{25} \mathrm{D}=+28.3(\mathrm{c}=0.0135 \mathrm{~g} / 2.0 \mathrm{~mL}$, chloroform $) .{ }^{1} \mathrm{H}$ NMR $\left(400 \mathrm{MHz}, \mathrm{CDCl}_{3}\right)$ : Signals corresponding to the major diastereomer $\delta 9.47(1 \mathrm{H}, \mathrm{s}), 5.02$ $(1 \mathrm{H}, \mathrm{tt}, \mathrm{J}=1.5,7.0 \mathrm{~Hz}), 4.39(1 \mathrm{H}, \mathrm{dd}, \mathrm{J}=3.3,12.1 \mathrm{~Hz}), 4.13(1 \mathrm{H}, \mathrm{dd}, \mathrm{J}=10.6,12.1 \mathrm{~Hz})$, $2.73(1 \mathrm{H}, \mathrm{m}), 1.88(2 \mathrm{H}, \mathrm{q}, \mathrm{J}=7.7 \mathrm{~Hz}), 1.67(3 \mathrm{H}, \mathrm{s}), 1.65-1.49(2 \mathrm{H}, \mathrm{m}), 1.57(3 \mathrm{H}, \mathrm{s}), 1.04$ $(3 \mathrm{H}, \mathrm{d}, \mathrm{J}=6.6 \mathrm{~Hz}), 1.03(3 \mathrm{H}, \mathrm{s})$. Peaks corresponding to the minor diastereomer $\delta 9.46$ $(1 \mathrm{H}, \mathrm{s}), 5.02(1 \mathrm{H}, \mathrm{tt}, \mathrm{J}=1.5,7.0 \mathrm{~Hz}), 4.49(1 \mathrm{H}, \mathrm{dd}, \mathrm{J}=3.7,12.1 \mathrm{~Hz}), 4.16(1 \mathrm{H}, \mathrm{dd}, \mathrm{J}=$ 10.6, $12.1 \mathrm{~Hz}), 2.73(1 \mathrm{H}, \mathrm{m}), 1.88(2 \mathrm{H}, \mathrm{q}, \mathrm{J}=7.7 \mathrm{~Hz}), 1.67(3 \mathrm{H}, \mathrm{s}), 1.65-1.49(2 \mathrm{H}, \mathrm{m})$, $1.57(3 \mathrm{H}, \mathrm{s}), 1.05(3 \mathrm{H}, \mathrm{s}), 0.99(3 \mathrm{H}, \mathrm{d}, \mathrm{J}=6.6 \mathrm{~Hz}) .{ }^{13} \mathrm{C}\left(100 \mathrm{MHz}, \mathrm{CDCl}_{3}\right)$ : Signals corresponding to the major diastereomer $\delta 204.6,133.1,122.8,78.7,50.7,34.8,34.6$, 25.6, 22.3, 17.7, 14.9, 12.2. Signals corresponding to the minor diastereomer $\delta 204.1$, 133.1, 122.8, 78.2, 50.7, 36.0, 34.2, 29.7, 25.6, 22.4, 14.8, 13.4. IR (neat): 2973 (s), 2924 (s), $2858(\mathrm{~m}), 2713$ (w), 1726 (s), 1554 (s), 1436 (m), 1378 (s), 1231 (w), $1119(\mathrm{w}), 902$ (w). HRMS (ESI): expected for $\left[\mathrm{C}_{12} \mathrm{H}_{21} \mathrm{NO}_{3}+\mathrm{H}\right]^{+}: 228.1599$, found: 228.1596 . 


\section{Racemic 2,6-dimethyl-2-(1-nitropropan-2-yl)hept-5-enal}

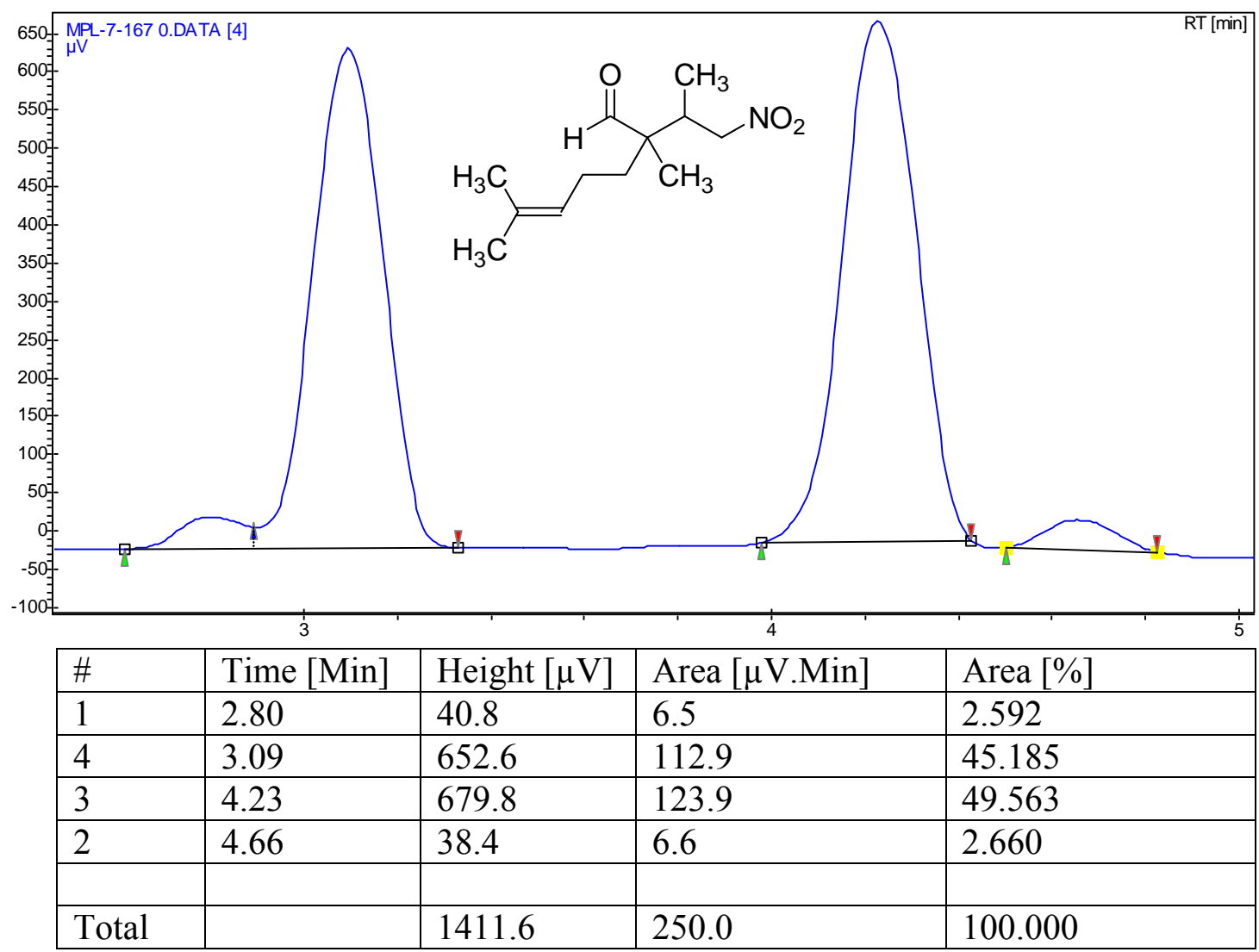


Enantioenriched $(R)$-2,6-dimethyl-2-((R)-1-nitropropan-2-yl)hept-5-enal $+(S)$-2,6dimethyl-2-((R)-1-nitropropan-2-yl)hept-5-enal

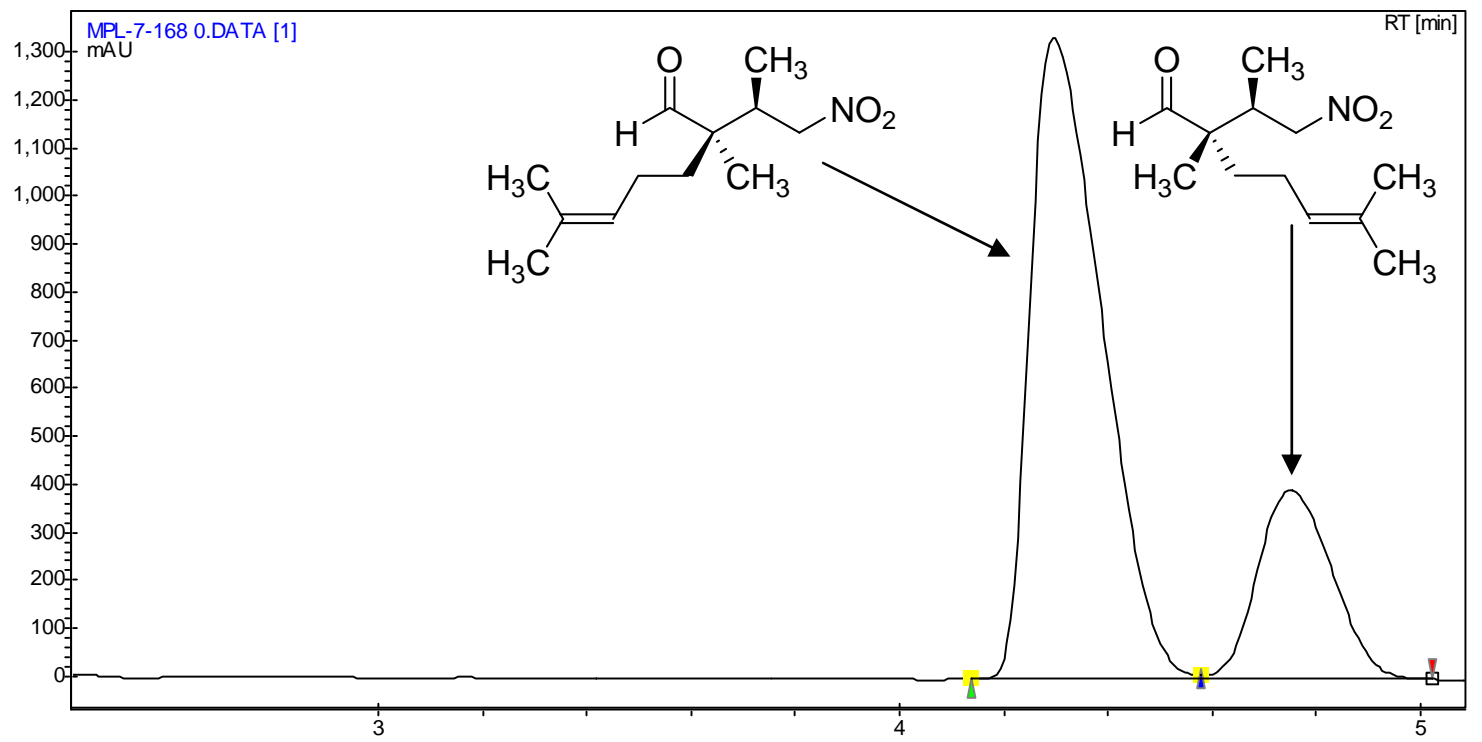

\begin{tabular}{|l|l|l|l|l|}
\hline$\#$ & Time $[\mathrm{Min}]$ & Height $[\mathrm{mAU}]$ & $\begin{array}{l}\text { Area } \\
{\left[\mathrm{mAU} \mathbf{m i n}^{*}\right]}\end{array}$ & Area [\%] \\
\hline 1 & 4.29 & 1336.73 & 212.96 & 76.532 \\
\hline 2 & 4.75 & 391.84 & 65.30 & 23.468 \\
\hline & & & & \\
\hline Total & & & 278.26 & 100.000 \\
\hline
\end{tabular}

(R)-2,6-dimethyl-2-((R)-2-nitro-1-phenylethyl)hept-5-enal (10): General procedure, diastereomeric products separable by chromatography on silica $(10 \%$ diethyl ether/hexanes), colorless to light yellow liquid, $82 \%$ yield $(238.7 \mathrm{mg}$ ), 3.9:1 dr, $99 \%$ ee (major diastereomer), 97\% ee (minor diastereomer) as determined by HPLC (Chiralcel $\mathrm{OD}-\mathrm{H}, 5.0 \%$ isopropanol/hexanes, $1.0 \mathrm{~mL} / \mathrm{min}, 208 \mathrm{~nm}$; $t_{r}$ (major enantiomer, major diastereomer $)=21.92 \mathrm{~min}, t_{r}($ minor enantiomer, major diastereomer $\left.)=28.63 \mathrm{~min}\right)$. $[\alpha]_{\mathrm{D}}^{25}=+55.9(\mathrm{c}=0.0210 \mathrm{~g} / 2.0 \mathrm{~mL}$, chloroform $) .{ }^{1} \mathrm{H}$ NMR $\left(400 \mathrm{MHz}, \mathrm{CDCl}_{3}\right): \delta 9.52$ $(1 \mathrm{H}, \mathrm{s}), 7.34-7.18(5 \mathrm{H}, \mathrm{m}), 4.90(1 \mathrm{H}, \mathrm{t}, \mathrm{J}=7.0 \mathrm{~Hz}), 4.81(1 \mathrm{H}, \mathrm{dd}, \mathrm{J}=11.3,12.8 \mathrm{~Hz}), 4.60$ $(1 \mathrm{H}, \mathrm{dd}, \mathrm{J}=3.8,12.8 \mathrm{~Hz}), 3.58(1 \mathrm{H}, \mathrm{dd}, \mathrm{J}=3.8,11.3 \mathrm{~Hz}), 1.84(2 \mathrm{H}, \mathrm{q}, \mathrm{J}=8.1 \mathrm{~Hz}), 1.61$ $(3 \mathrm{H}, \mathrm{s}), 1.55(1 \mathrm{H}, \mathrm{m}), 1.50(3 \mathrm{H}, \mathrm{s}), 1.20(1 \mathrm{H}, \mathrm{m}), 1.10(3 \mathrm{H}, \mathrm{s}) \cdot{ }^{13} \mathrm{C}\left(100 \mathrm{MHz}, \mathrm{CDCl}_{3}\right): \delta$ 205.0, 135.2, 133.0, 129.2, 128.7, 128.2, 122.8, 76.7, 51.5, 47.6, 35.5, 25.6, 22.4, 17.7, 15.7. IR (neat): $3033(\mathrm{~m}), 2972(\mathrm{~m}), 2920(\mathrm{~m}), 2856(\mathrm{~m}), 2724(\mathrm{~m}), 1723(\mathrm{~s}), 1556(\mathrm{~s})$, $1455(\mathrm{~m}), 1378(\mathrm{~s}), 750(\mathrm{~m}), 705$ (s). HRMS (ESI): expected for $\left[\mathrm{C}_{17} \mathrm{H}_{23} \mathrm{NO}_{3}+\mathrm{NH}_{4}\right]^{+}$: 307.2022, found: 307.2030 .

(S)-2,6-dimethyl-2-((R)-2-nitro-1-phenylethyl)hept-5-enal: $\quad 99 \%$ ee (minor diastereomer) as determined by HPLC (Chiralcel OD-H, 5.0\% ispropanol/hexanes, 1.0 $\mathrm{mL} / \mathrm{min}, 208 \mathrm{~nm}$; $t_{r}$ (major enantiomer, minor diastereomer $)=19.31 \mathrm{~min}, t_{r}($ minor 
enantiomer, minor diastereomer $)=32.42 \mathrm{~min}) \cdot[\alpha]^{25}{ }_{\mathrm{D}}=-1.3(\mathrm{c}=0.0580 \mathrm{~g} / 2.0 \mathrm{~mL}$, chloroform). ${ }^{1} \mathrm{H}$ NMR (400 MHz, $\left.\mathrm{CDCl}_{3}\right): \delta 9.53(1 \mathrm{H}, \mathrm{s}), 7.34-7.15(5 \mathrm{H}, \mathrm{m}), 4.99(1 \mathrm{H}, \mathrm{t}$, $\mathrm{J}=7.1 \mathrm{~Hz}), 4.84(1 \mathrm{H}, \mathrm{dd}, \mathrm{J}=11.3,13.2 \mathrm{~Hz}), 4.76(1 \mathrm{H}, \mathrm{dd}, \mathrm{J}=4.4,13.2 \mathrm{~Hz}), 3.76(1 \mathrm{H}$, dd, J = 4.4, $11.3 \mathrm{~Hz}), 1.97(1 \mathrm{H}, \mathrm{m}), 1.88(1 \mathrm{H}, \mathrm{m}), 1.68(1 \mathrm{H}, \mathrm{m}), 1.66(3 \mathrm{H}, \mathrm{s}), 1.56(3 \mathrm{H}$, s), $1.52(1 \mathrm{H}, \mathrm{m}), 1.13(3 \mathrm{H}, \mathrm{s}) .{ }^{13} \mathrm{C}\left(100 \mathrm{MHz}, \mathrm{CDCl}_{3}\right): \delta 204.8,135.2,133.0,129.1$, $128.8,128.2,122.9,76.3,50.9,49.4,34.4,25.6,22.6,17.7,17.2$. IR (neat): $3033(\mathrm{~m})$, $2969(\mathrm{~m}), 2922(\mathrm{~m}), 2854(\mathrm{~m}), 2722(\mathrm{~m}), 1724(\mathrm{~s}), 1556(\mathrm{~s}), 1455(\mathrm{~m}), 1379(\mathrm{~s}), 750(\mathrm{~m})$, 704 (s). HRMS (ESI): expected for $\left[\mathrm{C}_{17} \mathrm{H}_{23} \mathrm{NO}_{3}+\mathrm{NH}_{4}\right]^{+}$: 307.2022 , found: 307.2007 .

\section{Racemic 2,6-dimethyl-2-(2-nitro-1-phenylethyl)hept-5-enal}

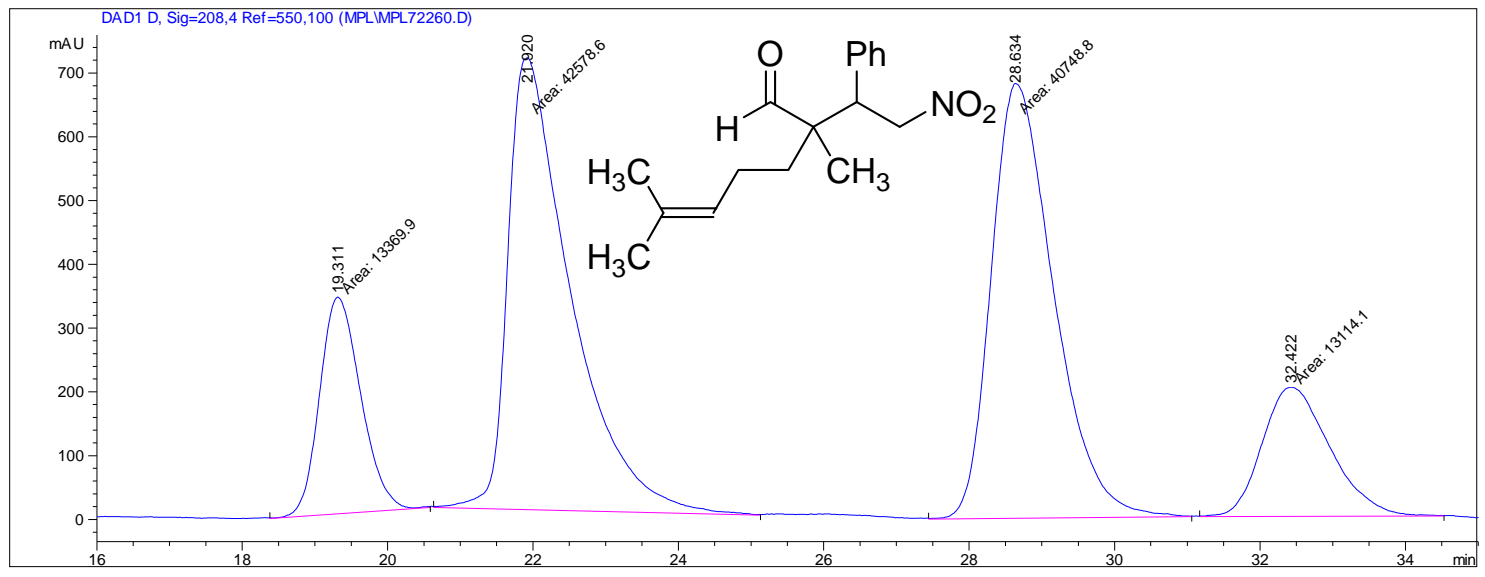

\begin{tabular}{|l|l|l|l|l|l|l|}
\hline $\begin{array}{l}\text { Peak } \\
\#\end{array}$ & $\begin{array}{l}\text { RetTime } \\
{[\mathrm{min}]}\end{array}$ & Type & $\begin{array}{l}\text { Width } \\
{[\mathrm{min}]}\end{array}$ & $\begin{array}{l}\text { Area } \\
{[\mathrm{mAU} * \mathrm{~s}]}\end{array}$ & $\begin{array}{l}\text { Height } \\
{[\mathrm{mAU}]}\end{array}$ & $\begin{array}{l}\text { Area } \\
{[\%]}\end{array}$ \\
\hline 1 & 19.311 & MM & 0.6552 & $1.33646 \mathrm{e} 4$ & 339.94751 & 12.2017 \\
\hline 2 & 21.920 & MM & 0.9991 & $4.24427 \mathrm{e} 4$ & 708.04962 & 38.7498 \\
\hline 3 & 28.634 & MM & 0.9932 & $4.06252 \mathrm{e} 4$ & 681.74896 & 37.0904 \\
\hline 4 & 32.422 & MM & 1.0794 & $1.30977 \mathrm{e} 4$ & 202.23651 & 11.9581 \\
\hline Totals: & & & & $1.09530 \mathrm{e} 5$ & 1931.98260 & \\
\hline
\end{tabular}


Enantioenriched $(R)$-2,6-dimethyl-2-((R)-2-nitro-1-phenylethyl)hept-5-enal + $(S)$ 2,6-dimethyl-2-((R)-2-nitro-1-phenylethyl)hept-5-enal

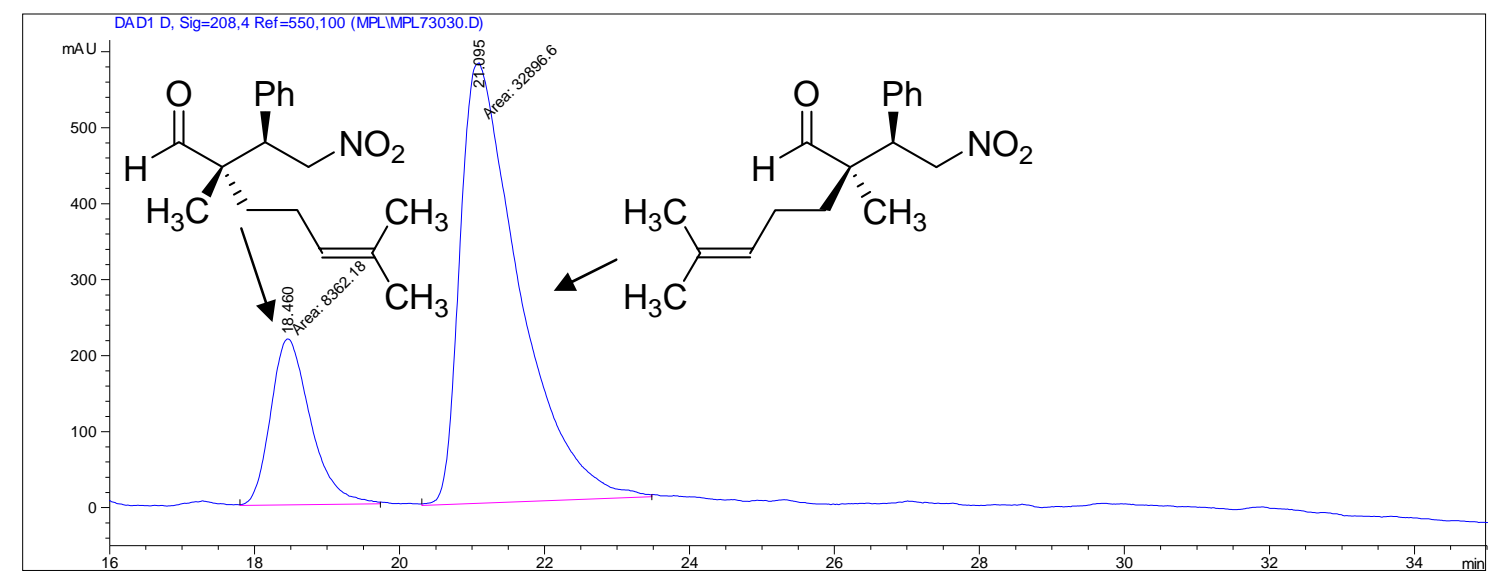

\begin{tabular}{|l|l|l|l|l|l|l|}
\hline $\begin{array}{l}\text { Peak } \\
\#\end{array}$ & $\begin{array}{l}\text { RetTime } \\
{[\mathrm{min}]}\end{array}$ & Type & $\begin{array}{l}\text { Width } \\
{[\mathrm{min}]}\end{array}$ & $\begin{array}{l}\text { Area } \\
{\left[\mathrm{mAU}^{*} \mathrm{~s}\right]}\end{array}$ & $\begin{array}{l}\text { Height } \\
{[\mathrm{mAU}]}\end{array}$ & $\begin{array}{l}\text { Area } \\
{[\%]}\end{array}$ \\
\hline 1 & 18.460 & $\mathrm{MM}$ & 0.6623 & 8759.28125 & 220.44008 & 20.2295 \\
\hline 2 & 21.095 & $\mathrm{MM}$ & 0.9880 & $3.45403 \mathrm{e} 4$ & 582.65924 & 79.7705 \\
\hline Totals: & & & & $4.32996 \mathrm{e} 4$ & 803.09932 & \\
\hline
\end{tabular}

(2S,3R)-2-((tert-butyldimethylsilyloxy)methyl)-2-methyl-4-nitro-3-phenylbutanal (11): General procedure, diastereomeric products separable by chromatography on silica (8\% diethyl ether/hexanes), colorless to light yellow oil, $87 \%$ yield $(306.3 \mathrm{mg}$ ), 6.3:1 dr (determined using the HPLC conditions below and the crude product mixture), $99 \%$ ee (major diastereomer) as determined by HPLC (Chiralcel OD-H, $0.5 \%$ isopropanol/hexanes, $1.0 \mathrm{~mL} / \mathrm{min}, 208 \mathrm{~nm}$; $t_{r}($ major enantiomer, major diastereomer $)=$ $16.54 \mathrm{~min}, t_{r}($ minor enantiomer, major diastereomer $\left.)=17.62 \mathrm{~min}\right) .[\alpha]^{25}=+18.9(\mathrm{c}=$ $0.0203 \mathrm{~g} / 2.0 \mathrm{~mL}$, chloroform). ${ }^{1} \mathrm{H}$ NMR $\left(400 \mathrm{MHz}, \mathrm{CDCl}_{3}\right): \delta 9.61(1 \mathrm{H}, \mathrm{s}), 7.32-7.24$ $(5 \mathrm{H}, \mathrm{m}), 4.90(1 \mathrm{H}, \mathrm{dd}, \mathrm{J}=11.3,13.2 \mathrm{~Hz}), 4.80(1 \mathrm{H}, \mathrm{dd}, \mathrm{J}=4.0,13.2 \mathrm{~Hz}), 4.13(1 \mathrm{H}, \mathrm{dd}, \mathrm{J}$ $=4.0,11.3 \mathrm{~Hz}), 3.49(1 \mathrm{H}, \mathrm{d}, \mathrm{J}=10.2 \mathrm{~Hz}), 3.34(1 \mathrm{H}, \mathrm{d}, \mathrm{J}=10.2 \mathrm{~Hz}), 1.04(3 \mathrm{H}, \mathrm{s}), 0.90$ (9H, s), $0.01(6 \mathrm{H}, \mathrm{s}) .{ }^{13} \mathrm{C}\left(100 \mathrm{MHz}, \mathrm{CDCl}_{3}\right): \delta 204.0,135.2,129.1,128.6,128.1,76.4$, 53.1, 44.4, 25.7, 18.1, 13.7, -5.7, -5.8. IR (neat): $3066(\mathrm{~m}), 3035(\mathrm{~m}), 2955,(\mathrm{~s}), 2930(\mathrm{~s})$, $2897(\mathrm{~m}), 2858(\mathrm{~s}), 2712(\mathrm{~m}), 1730(\mathrm{~s}), 1556(\mathrm{~s}), 1472(\mathrm{~m}), 1378(\mathrm{~m}), 1259(\mathrm{~m}), 1099(\mathrm{~s})$, 834 (s), 779 (s), 731 (s). HRMS (ESI): expected for $\left[\mathrm{C}_{18} \mathrm{H}_{29} \mathrm{NO}_{4} \mathrm{Si}+\mathrm{NH}_{4}\right]^{+}: 369.2210$, found: 369.2216 . 
Racemic 2-((tert-butyldimethylsilyloxy)methyl)-2-methyl-4-nitro-3-phenylbutanal

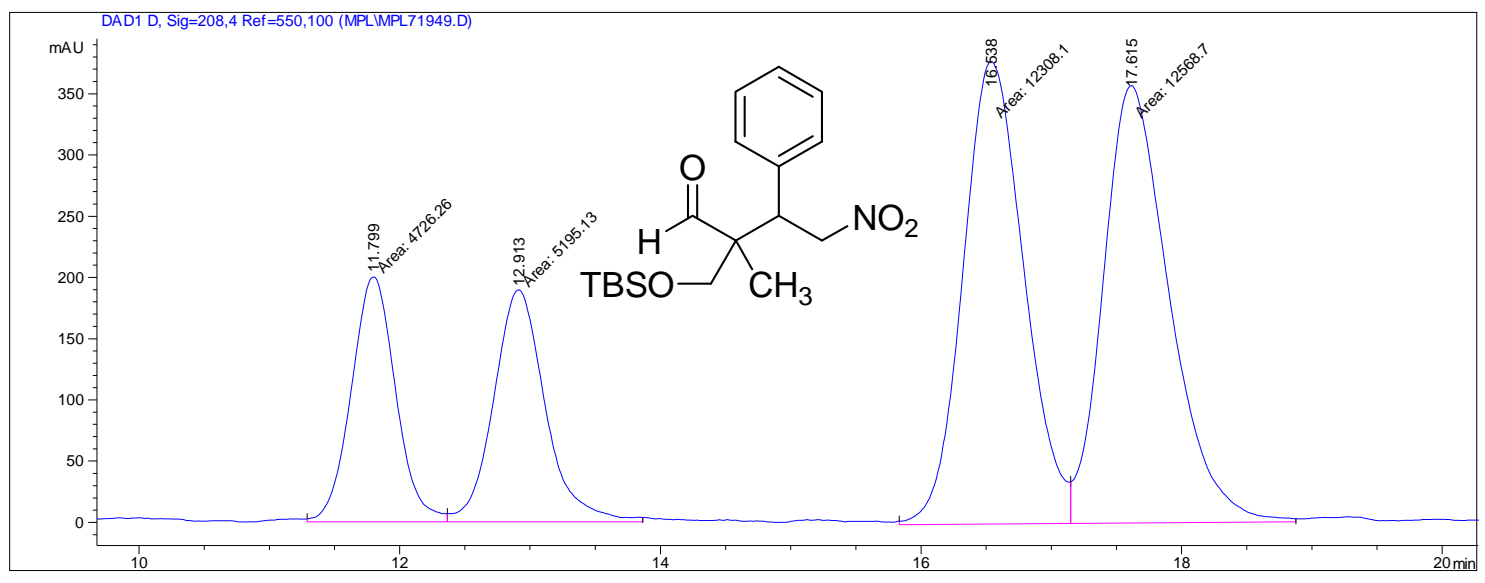

\begin{tabular}{|l|l|l|l|l|l|l|}
\hline $\begin{array}{l}\text { Peak } \\
\#\end{array}$ & $\begin{array}{l}\text { RetTime } \\
{[\mathrm{min}]}\end{array}$ & Type & $\begin{array}{l}\text { Width } \\
{[\mathrm{min}]}\end{array}$ & $\begin{array}{l}\text { Area } \\
{[\mathrm{mAU} * \mathrm{~s}]}\end{array}$ & $\begin{array}{l}\text { Height } \\
{[\mathrm{mAU}]}\end{array}$ & $\begin{array}{l}\text { Area } \\
{[\%]}\end{array}$ \\
\hline 1 & 11.799 & MF & 0.3911 & 4669.92187 & 199.00484 & 13.5112 \\
\hline 2 & 12.913 & FM & 0.4519 & 5106.52588 & 188.35178 & 14.7743 \\
\hline 3 & 16.538 & MF & 0.5418 & $1.22711 \mathrm{e} 4$ & 377.48682 & 35.5030 \\
\hline 4 & 17.615 & FM & 0.5850 & $1.25159 \mathrm{e} 4$ & 356.55698 & 36.2115 \\
\hline Totals: & & & & $3.45635 \mathrm{e} 4$ & 1121.40041 & \\
\hline
\end{tabular}

Enantioenriched (2S,3R)-2-((tert-butyldimethylsilyloxy)methyl)-2-methyl-4-nitro-3phenylbutanal

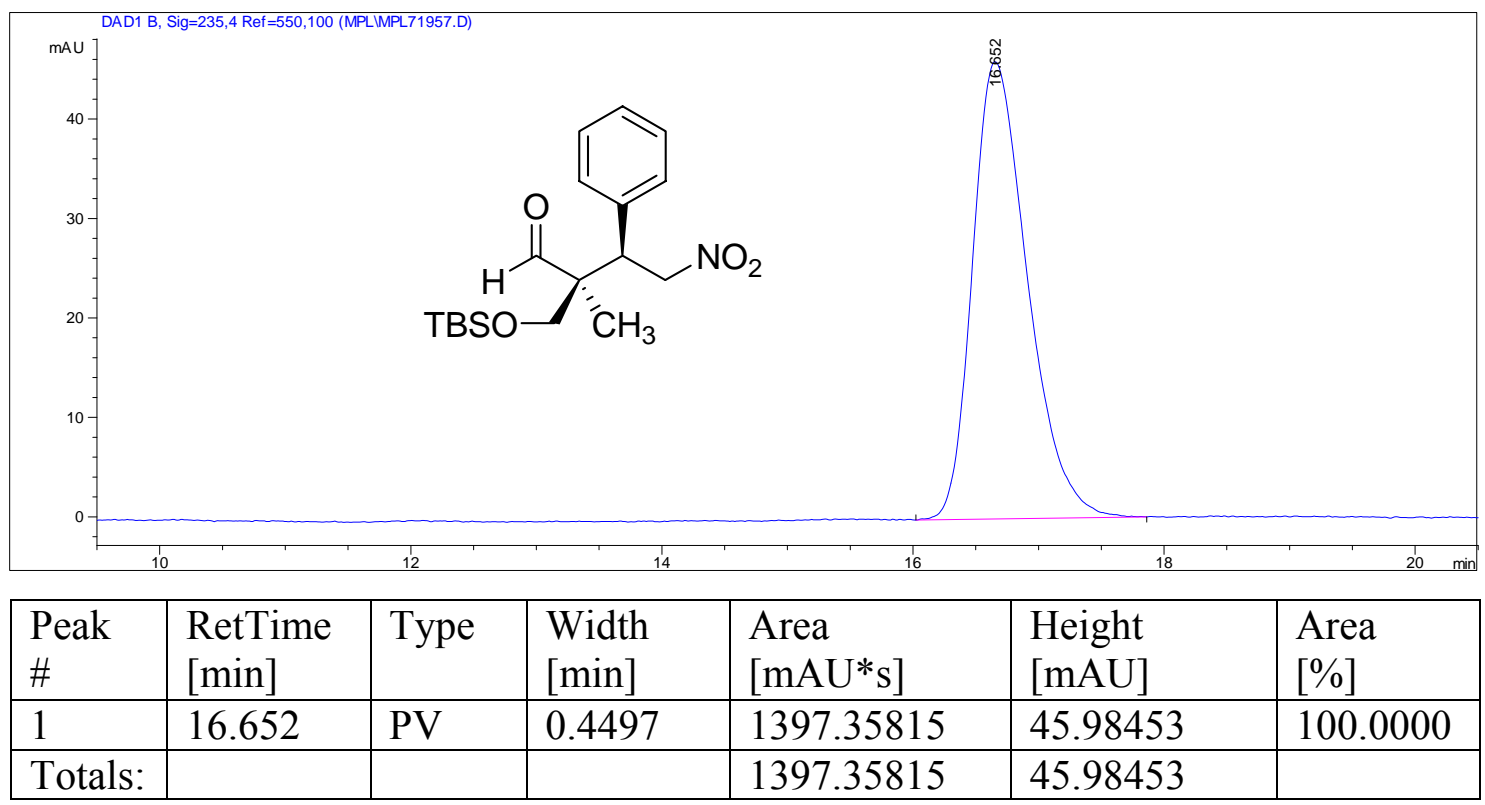


(2R,3R)-2-((tert-butyldimethylsilyloxy)methyl)-2-methyl-4-nitro-3-phenylbutanal:

white solid, m.p. $=79-80{ }^{\circ} \mathrm{C}, 96 \%$ ee (minor diastereomer) as determined by HPLC (Chiralcel OD-H, 0.5\% isopropanol/hexanes, $1.0 \mathrm{~mL} / \mathrm{min}, 235 \mathrm{~nm}$; $t_{r}$ (major enantiomer, minor diastereomer $)=11.80 \mathrm{~min}, t_{r}($ minor enantiomer, minor diastereomer $)=12.91$ $\min ) \cdot[\alpha]_{\mathrm{D}}^{25}=-24.5(\mathrm{c}=0.0103 \mathrm{~g} / 2.0 \mathrm{~mL}$, chloroform $) .{ }^{1} \mathrm{H}$ NMR $\left(400 \mathrm{MHz}, \mathrm{CDCl}_{3}\right): \delta$ $9.61(1 \mathrm{H}, \mathrm{s}), 7.35-7.23(5 \mathrm{H}, \mathrm{m}), 5.08(1 \mathrm{H}, \mathrm{dd}, \mathrm{J}=11.7,13.5 \mathrm{~Hz}), 4.83(1 \mathrm{H}, \mathrm{dd}, \mathrm{J}=3.3$, $13.5 \mathrm{~Hz}), 4.01(1 \mathrm{H}, \mathrm{dd}, \mathrm{J}=3.3,11.7 \mathrm{~Hz}), 3.74(1 \mathrm{H}, \mathrm{d}, \mathrm{J}=11.0 \mathrm{~Hz}), 3.65(1 \mathrm{H}, \mathrm{d}, \mathrm{J}=11.0$ $\mathrm{Hz}), 0.94(9 \mathrm{H}, \mathrm{s}), 0.82(3 \mathrm{H}, \mathrm{s}), 0.14(3 \mathrm{H}, \mathrm{s}), 0.10(3 \mathrm{H}, \mathrm{s}) .{ }^{13} \mathrm{C}\left(100 \mathrm{MHz}, \mathrm{CDCl}_{3}\right): \delta$ 203.5, 135.6, 129.3, 128.7, 128.0, 77.0, 64.7, 53.5, 46.7, 25.8, 18.1, 17.0, -5.6, -5.7. IR (thin film): 3066 (m), 3035 (m), 2956 (s), 2930 (s), 2897 (m), 2858 (s), 2715 (m), 1726 (s), $1555(\mathrm{~s}), 1472(\mathrm{~m}), 1378(\mathrm{~m}), 1259(\mathrm{~m}), 1090(\mathrm{~s}), 838(\mathrm{~s}), 779$ (s), 705 (s). HRMS (ESI): expected for $\left[\mathrm{C}_{18} \mathrm{H}_{29} \mathrm{NO}_{4} \mathrm{Si}+\mathrm{NH}_{4}\right]^{+}: 369.2210$, found: 369.2209 .

\section{Enantioenriched (2R,3R)-2-((tert-butyldimethylsilyloxy)methyl)-2-methyl-4-nitro-3- phenylbutanal}

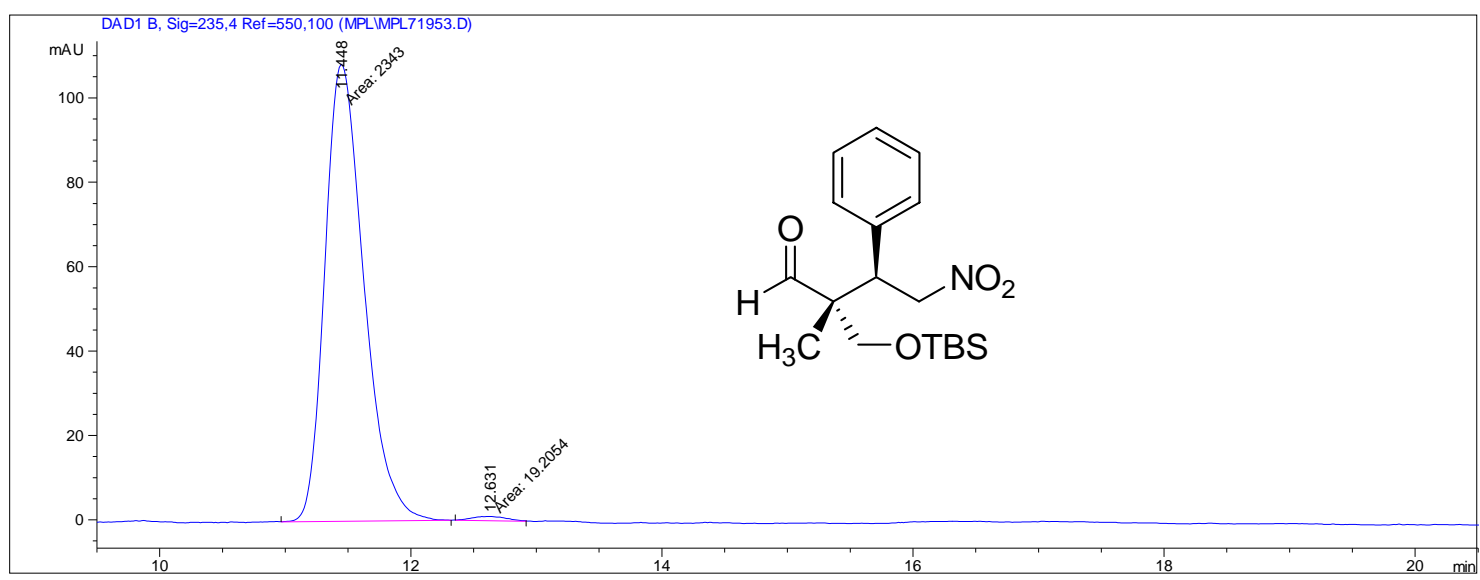

\begin{tabular}{|l|l|l|l|l|l|l|}
\hline $\begin{array}{l}\text { Peak } \\
\#\end{array}$ & $\begin{array}{l}\text { RetTime } \\
{[\mathrm{min}]}\end{array}$ & Type & $\begin{array}{l}\text { Width } \\
{[\mathrm{min}]}\end{array}$ & $\begin{array}{l}\text { Area } \\
{\left[\mathrm{mAU}^{*} \mathrm{~s}\right]}\end{array}$ & $\begin{array}{l}\text { Height } \\
{[\mathrm{mAU}]}\end{array}$ & $\begin{array}{l}\text { Area } \\
{[\%]}\end{array}$ \\
\hline 1 & 11.448 & $\mathrm{MM}$ & 0.3598 & 2340.93750 & 108.44026 & 99.1828 \\
\hline 2 & 12.631 & $\mathrm{MM}$ & 0.3190 & 19.28873 & 1.00766 & 0.8172 \\
\hline Totals: & & & & 2360.22623 & 109.44792 & \\
\hline
\end{tabular}

(2S,3R)-2-((tert-butyldimethylsilyloxy)methyl)-3-(4-fluorophenyl)-2-methyl-4-

nitrobutanal (12): General procedure, diastereomeric products separable by chromatography on silica ( $15 \%$ diethyl ether/hexanes), white solid, m.p.(major diastereomer) $=59-61{ }^{\circ} \mathrm{C}, 86 \%$ yield $(318.6 \mathrm{mg}), 6.6: 1 \mathrm{dr}$ (determined using the HPLC conditions below and the crude product mixture), $99 \%$ ee (major diastereomer) as 
determined by HPLC (Econosphere $\mathrm{CN} 5 \mathrm{U}+$ Chiralpak AD-H, 0.5\% isopropanol/hexanes, $1.0 \mathrm{~mL} / \mathrm{min}, 208 \mathrm{~nm}$; $t_{r}($ minor enantiomer, major diastereomer $)=$ $18.15 \mathrm{~min}, t_{r}$ (major enantiomer, major diastereomer $\left.)=19.75 \mathrm{~min}\right) .[\alpha]^{25}{ }_{\mathrm{D}}=+15.9(\mathrm{c}=$ $0.0202 \mathrm{~g} / 2.0 \mathrm{~mL}$, chloroform). ${ }^{1} \mathrm{H}$ NMR $\left(400 \mathrm{MHz}, \mathrm{CDCl}_{3}\right): \delta 9.58(1 \mathrm{H}, \mathrm{s}), 7.24(2 \mathrm{H}, \mathrm{dd}$, $\mathrm{J}=5.3,8.8 \mathrm{~Hz}), 7.00(2 \mathrm{H}, \mathrm{t}, \mathrm{J}=8.8 \mathrm{~Hz}), 4.84(2 \mathrm{H}, \mathrm{m}), 4.12(1 \mathrm{H}, \mathrm{dd}, \mathrm{J}=4.9,10.6 \mathrm{~Hz})$, $3.52(1 \mathrm{H}, \mathrm{d}, \mathrm{J}=10.2 \mathrm{~Hz}), 3.30(1 \mathrm{H}, \mathrm{d}, \mathrm{J}=10.2 \mathrm{~Hz}), 1.02(3 \mathrm{H}, \mathrm{s}), 0.90(9 \mathrm{H}, \mathrm{s}), 0.02(6 \mathrm{H}$, s). ${ }^{13} \mathrm{C}\left(100 \mathrm{MHz}, \mathrm{CDCl}_{3}\right): \delta 203.6,162.3(1 \mathrm{C}, \mathrm{d}, \mathrm{J}=247.2 \mathrm{~Hz}), 131.1(1 \mathrm{C}, \mathrm{d}, \mathrm{J}=3.8$ $\mathrm{Hz}), 130.7(1 \mathrm{C}, \mathrm{d}, \mathrm{J}=7.6 \mathrm{~Hz}), 115.5(1 \mathrm{C}, \mathrm{d}, \mathrm{J}=21.4 \mathrm{~Hz}), 76.5,65.8,53.3,43.8,25.7$, 18.1, 13.6, -5.7, -5.8. IR (thin film): 2955 (s), 2930 (s), 2858 (s), 2712 (m), 1727 (s), $1607(\mathrm{~m}), 1556(\mathrm{~s}), 1512(\mathrm{~s}), 1472(\mathrm{~m}), 1378(\mathrm{~m}), 1254(\mathrm{~m}), 1229(\mathrm{~m}), 1163(\mathrm{~m}), 1104$ (s), 840 (s), 779 (s). HRMS (ESI): expected for $\left[\mathrm{C}_{18} \mathrm{H}_{28} \mathrm{FNO}_{4} \mathrm{Si}\right]^{+}: 369.1772$, found: 369.1786 .

\section{Racemic 2-((tert-butyldimethylsilyloxy)methyl)-3-(4-fluorophenyl)-2-methyl-4- nitrobutanal}

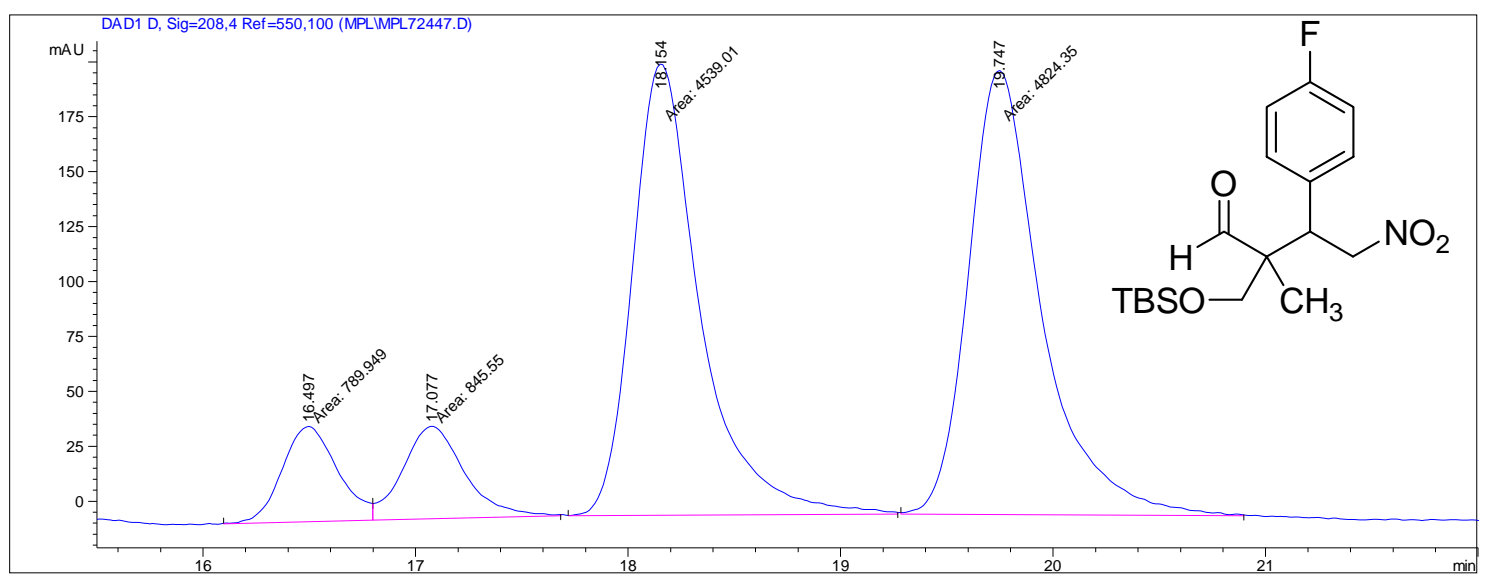

\begin{tabular}{|l|l|l|l|l|l|l|}
\hline $\begin{array}{l}\text { Peak } \\
\#\end{array}$ & $\begin{array}{l}\text { RetTime } \\
{[\mathrm{min}]}\end{array}$ & Type & $\begin{array}{l}\text { Width } \\
{[\mathrm{min}]}\end{array}$ & $\begin{array}{l}\text { Area } \\
{[\mathrm{mAU} \text { s }]}\end{array}$ & $\begin{array}{l}\text { Height } \\
{[\mathrm{mAU}]}\end{array}$ & $\begin{array}{l}\text { Area } \\
{[\%]}\end{array}$ \\
\hline 1 & 16.497 & MF & 0.3072 & 801.61078 & 43.49492 & 7.3643 \\
\hline 2 & 17.077 & FM & 0.3290 & 831.22321 & 42.10474 & 7.6363 \\
\hline 3 & 18.154 & MM & 0.3652 & 4499.10645 & 205.32422 & 41.3325 \\
\hline 4 & 19.747 & MM & 0.3931 & 4753.20898 & 201.53207 & 43.6669 \\
\hline Totals: & & & & $1.08851 \mathrm{e} 4$ & 492.45595 & \\
\hline
\end{tabular}


Enantioenriched (2S,3R)-2-((tert-butyldimethylsilyloxy)methyl)-3-(4-fluorophenyl)2-methyl-4-nitrobutanal

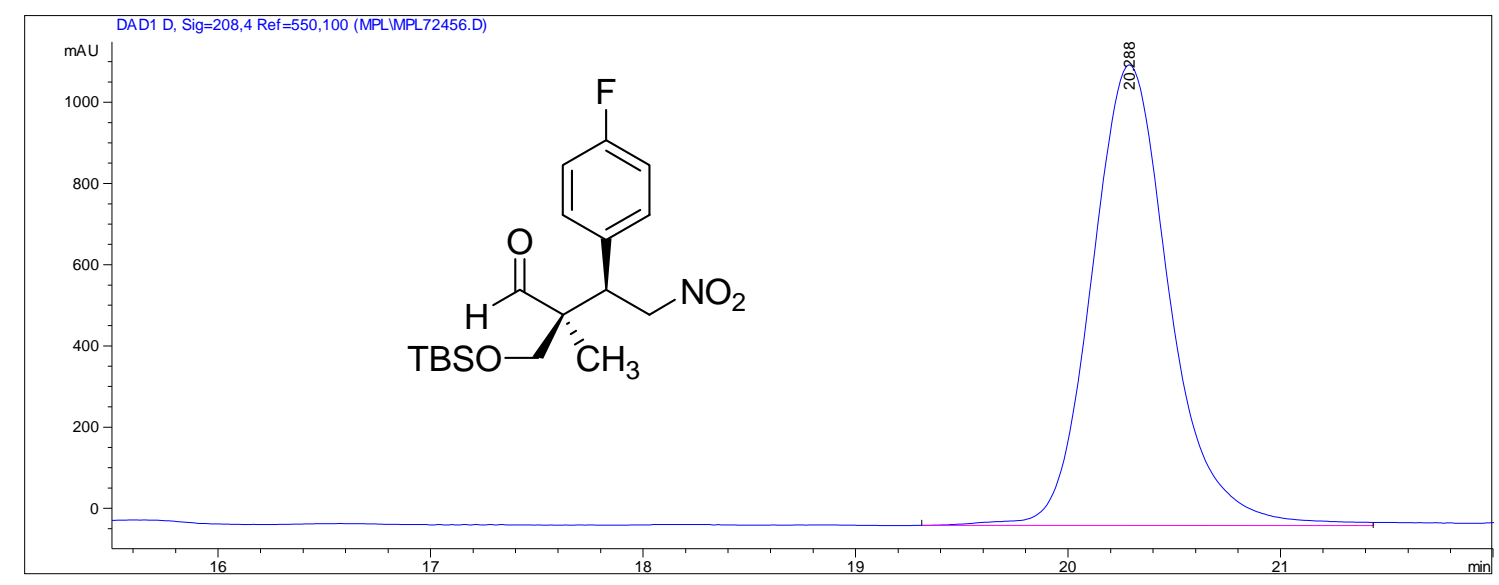

\begin{tabular}{|l|l|l|l|l|l|l|}
\hline $\begin{array}{l}\text { Peak } \\
\#\end{array}$ & $\begin{array}{l}\text { RetTime } \\
{[\mathrm{min}]}\end{array}$ & Type & $\begin{array}{l}\text { Width } \\
{[\mathrm{min}]}\end{array}$ & $\begin{array}{l}\text { Area } \\
{\left[\mathrm{mAU}^{*} \mathrm{~s}\right]}\end{array}$ & $\begin{array}{l}\text { Height } \\
{[\mathrm{mAU}]}\end{array}$ & $\begin{array}{l}\text { Area } \\
{[\%]}\end{array}$ \\
\hline 1 & 20.288 & $\mathrm{MM}$ & 0.4161 & $2.81835 \mathrm{e} 4$ & 1128.84021 & 100.0000 \\
\hline Totals: & & & & $2.81835 \mathrm{e} 4$ & 1128.84021 & \\
\hline
\end{tabular}

(2R,3R)-2-((tert-butyldimethylsilyloxy)methyl)-3-(4-fluorophenyl)-2-methyl-4-

nitrobutanal: Colorless to light yellow liquid, $94 \%$ ee (minor diastereomer) as determined by HPLC (Econosphere $\mathrm{CN} 5 \mathrm{U}+$ Chiralpak AD-H, $0.5 \%$ isopropanol/hexanes, $1.0 \mathrm{~mL} / \mathrm{min}, 208 \mathrm{~nm} ; t_{r}$ (major enantiomer, minor diastereomer $)=$ $16.50 \mathrm{~min}, t_{r}($ minor enantiomer, minor diastereomer $\left.)=17.08 \mathrm{~min}\right) .[\alpha]_{\mathrm{D}}^{25}=-22.5(\mathrm{c}=$ $0.0296 \mathrm{~g} / 2.0 \mathrm{~mL}$, chloroform). ${ }^{1} \mathrm{H}$ NMR $\left(400 \mathrm{MHz}, \mathrm{CDCl}_{3}\right): \delta 9.59(1 \mathrm{H}, \mathrm{s}), 7.22(2 \mathrm{H}, \mathrm{dd}$, $\mathrm{J}=5.3,8.8 \mathrm{~Hz}), 7.03(2 \mathrm{H}, \mathrm{t}, \mathrm{J}=8.8 \mathrm{~Hz}), 5.03(1 \mathrm{H}, \mathrm{dd}, \mathrm{J}=11.7,13.5 \mathrm{~Hz}), 4.82(1 \mathrm{H}, \mathrm{dd}, \mathrm{J}$ $=3.7,13.5 \mathrm{~Hz}), 4.01(1 \mathrm{H}, \mathrm{dd}, \mathrm{J}=3.7,11.7 \mathrm{~Hz}), 3.74(1 \mathrm{H}, \mathrm{d}, \mathrm{J}=11.2 \mathrm{~Hz}), 3.61(1 \mathrm{H}, \mathrm{d}$, $11.2 \mathrm{~Hz}), 0.93(9 \mathrm{H}, \mathrm{s}), 0.82(3 \mathrm{H}, \mathrm{s}), 0.14(3 \mathrm{H}, \mathrm{s}), 0.10(3 \mathrm{H}, \mathrm{s}) .{ }^{13} \mathrm{C}\left(100 \mathrm{MHz}, \mathrm{CDCl}_{3}\right): \delta$ 203.2, $162.4(1 \mathrm{C}, \mathrm{d}, \mathrm{J}=248.0 \mathrm{~Hz}), 131.3(1 \mathrm{C}, \mathrm{d}, \mathrm{J}=3.1 \mathrm{~Hz}), 130.9(1 \mathrm{C}, \mathrm{d}, \mathrm{J}=8.4 \mathrm{~Hz})$, $115.7(1 \mathrm{C}, \mathrm{d}, \mathrm{J}=21.4 \mathrm{~Hz}), 77.0,64.6,53.5,46.1,25.8,18.1,16.9,-5.6,-5.7$. IR (neat): 2955 (s), 2930 (s), 2858 (s), 2715 (m), 1729 (s), 1607 (m), 1555 (s), 1512 (s), 1378 (m), 1100 (s), 838 (s). HRMS (ESI): expected for $\left[\mathrm{C}_{18} \mathrm{H}_{28} \mathrm{FNO}_{4} \mathrm{Si}+\mathrm{H}\right]^{+}: 370.1850$, found: 370.1859 . 
Enantioenriched (2R,3R)-2-((tert-butyldimethylsilyloxy)methyl)-3-(4-fluorophenyl)2-methyl-4-nitrobutanal

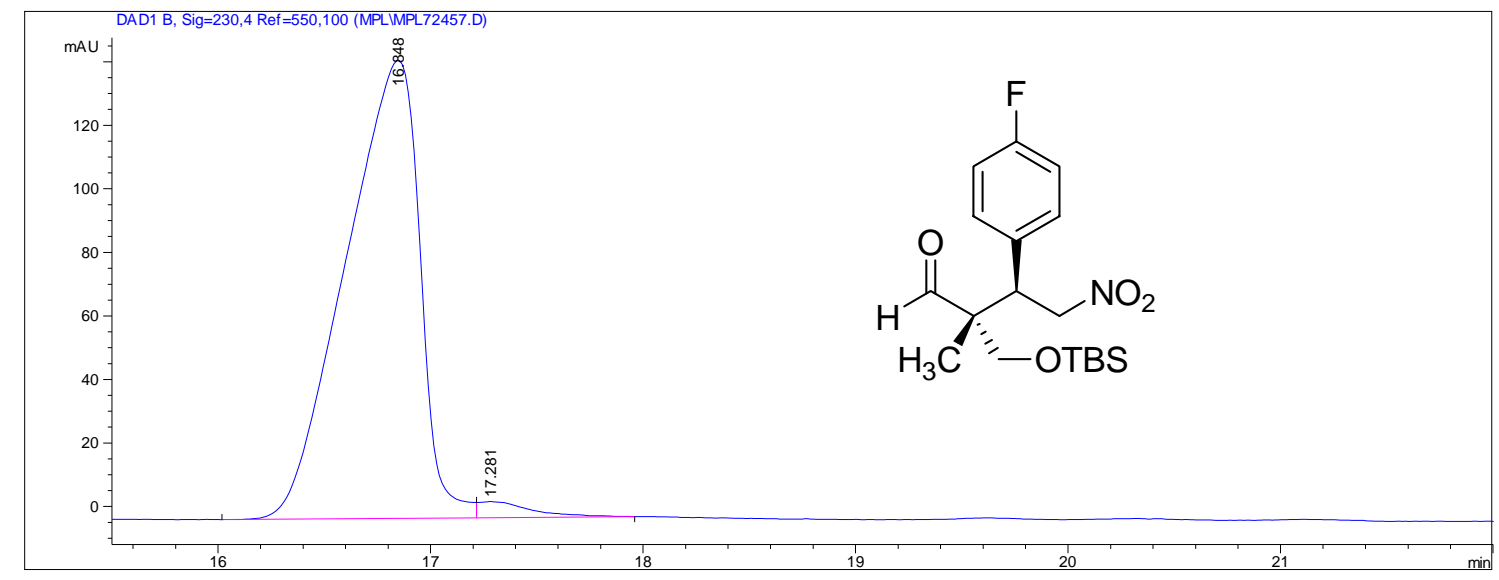

\begin{tabular}{|l|l|l|l|l|l|l|}
\hline $\begin{array}{l}\text { Peak } \\
\#\end{array}$ & $\begin{array}{l}\text { RetTime } \\
{[\mathrm{min}]}\end{array}$ & Type & $\begin{array}{l}\text { Width } \\
{[\mathrm{min}]}\end{array}$ & $\begin{array}{l}\text { Area } \\
{\left[\mathrm{mAU}{ }^{*} \mathrm{~s}\right]}\end{array}$ & $\begin{array}{l}\text { Height } \\
{[\mathrm{mAU}]}\end{array}$ & $\begin{array}{l}\text { Area } \\
{[\%]}\end{array}$ \\
\hline 1 & 16.848 & MF & 0.4034 & 3495.52808 & 144.41087 & 97.2581 \\
\hline 2 & 17.281 & FM & 0.3138 & 98.54705 & 5.23440 & 2.7419 \\
\hline Totals: & & & & 3594.07513 & 149.64527 & \\
\hline
\end{tabular}

(2S,3R)-2-((tert-butyldimethylsilyloxy)methyl)-3-(4-methoxyphenyl)-2-methyl-4nitrobutanal + (2R,3R)-2-((tert-butyldimethylsilyloxy)methyl)-3-(4-methoxyphenyl)2-methyl-4-nitrobutanal (13): General procedure, chromatography on silica (15\% diethyl ether/hexanes) diastereomers could not be separated, colorless to light yellow oil, $85 \%$ yield (325.4 mg), 7.1:1 dr, 99\% ee (major diastereomer), $95 \%$ ee (minor diastereomer) as determined by HPLC (Chiralcel OD-H, 1.0\% ethanol/hexanes, 1.0 $\mathrm{mL} / \mathrm{min}, 230 \mathrm{~nm}$; $t_{r}$ (minor enantiomer, minor diastereomer $)=12.57 \mathrm{~min}, t_{r}($ major enantiomer, minor diastereomer $)=13.99 \mathrm{~min}, t_{r}($ minor enantiomer, major diastereomer $)$ $=17.19 \mathrm{~min}, t_{r}($ major enantiomer, major diastereomer $\left.)=19.86 \mathrm{~min}\right) .[\alpha]^{25}=+9.0(\mathrm{c}=$ $0.0212 \mathrm{~g} / 2.0 \mathrm{~mL}$, chloroform). ${ }^{1} \mathrm{H}$ NMR ( $400 \mathrm{MHz}, \mathrm{CDCl}_{3}$ ): Signals corresponding to the major diastereomer $\delta 9.60(1 \mathrm{H}, \mathrm{s}), 7.16(2 \mathrm{H}, \mathrm{d}, \mathrm{J}=8.8 \mathrm{~Hz}), 6.83(2 \mathrm{H}, \mathrm{d}, \mathrm{J}=8.8 \mathrm{~Hz}), 4.85$ $(1 \mathrm{H}, \mathrm{dd}, \mathrm{J}=11.7,13.2 \mathrm{~Hz}), 4.76(1 \mathrm{H}, \mathrm{dd}, \mathrm{J}=4.2,13.2 \mathrm{~Hz}), 4.06(1 \mathrm{H}, \mathrm{dd}, \mathrm{J}=4.2,11.7$ $\mathrm{Hz}), 3.77(3 \mathrm{H}, \mathrm{s}), 3.48(1 \mathrm{H}, \mathrm{d}, \mathrm{J}=10.4 \mathrm{~Hz}), 3.36(1 \mathrm{H}, \mathrm{d}, \mathrm{J}=10.4 \mathrm{~Hz}), 1.03(3 \mathrm{H}, \mathrm{s}), 0.90$ $(9 \mathrm{H}, \mathrm{s}), 0.02(3 \mathrm{H}, \mathrm{s}), 0.01(3 \mathrm{H}, \mathrm{s})$. Signals corresponding to the minor diastereomer $\delta$ $9.60(1 \mathrm{H}, \mathrm{s}), 7.12(2 \mathrm{H}, \mathrm{d}, \mathrm{J}=8.8 \mathrm{~Hz}), 6.85(2 \mathrm{H}, \mathrm{d}, \mathrm{J}=8.8 \mathrm{~Hz}), 5.02(1 \mathrm{H}, \mathrm{dd}, \mathrm{J}=11.7$, $13.2 \mathrm{~Hz}), 4.82(1 \mathrm{H}, \mathrm{dd}, \mathrm{J}=3.7,13.2 \mathrm{~Hz}), 3.95(1 \mathrm{H}, \mathrm{dd}, \mathrm{J}=3.7,11.7 \mathrm{~Hz}), 3.77(3 \mathrm{H}, \mathrm{s})$, $3.72(1 \mathrm{H}, \mathrm{d}, \mathrm{J}=11.0 \mathrm{~Hz}), 3.63(1 \mathrm{H}, \mathrm{d}, \mathrm{J}=11.0 \mathrm{~Hz}), 0.93(9 \mathrm{H}, \mathrm{s}), 0.81(3 \mathrm{H}, \mathrm{s}), 0.13(3 \mathrm{H}$, s), $0.09(3 \mathrm{H}, \mathrm{s}) .{ }^{13} \mathrm{C}\left(100 \mathrm{MHz}, \mathrm{CDCl}_{3}\right)$ : Signals corresponding to the major diastereomer $\delta 204.2,159.2,130.1,127.0,113.9,76.5,65.8,55.1,53.4,43.8,25.7,18.1,13.6,-5.7$, 5.8. Signals corresponding to the minor diastereomer $\delta 203.7,130.3,127.2,114.0,77.1$, 64.7, 55.0, 53.6, 46.0, 25.6, 18.1, 16.8, -5.6, -5.7. IR (neat): 2956 (s), 2931 (s), 2857 (s), $2711(\mathrm{~m}), 1727$ (s), $1612(\mathrm{~m}), 1559(\mathrm{~s}), 1515(\mathrm{~s}), 1471(\mathrm{~m}), 1378(\mathrm{~m}), 1253(\mathrm{~s}), 1097(\mathrm{~s})$, 
$1036(\mathrm{~m}), 838$ (s), 779 (m). HRMS (ESI): expected for $\left[\mathrm{C}_{19} \mathrm{H}_{31} \mathrm{NO}_{5} \mathrm{Si}+\mathrm{NH}_{4}\right]^{+}: 399.2315$, found: 399.2311 .

Racemic 2-((tert-butyldimethylsilyloxy)methyl)-3-(4-methoxyphenyl)-2-methyl-4nitrobutanal

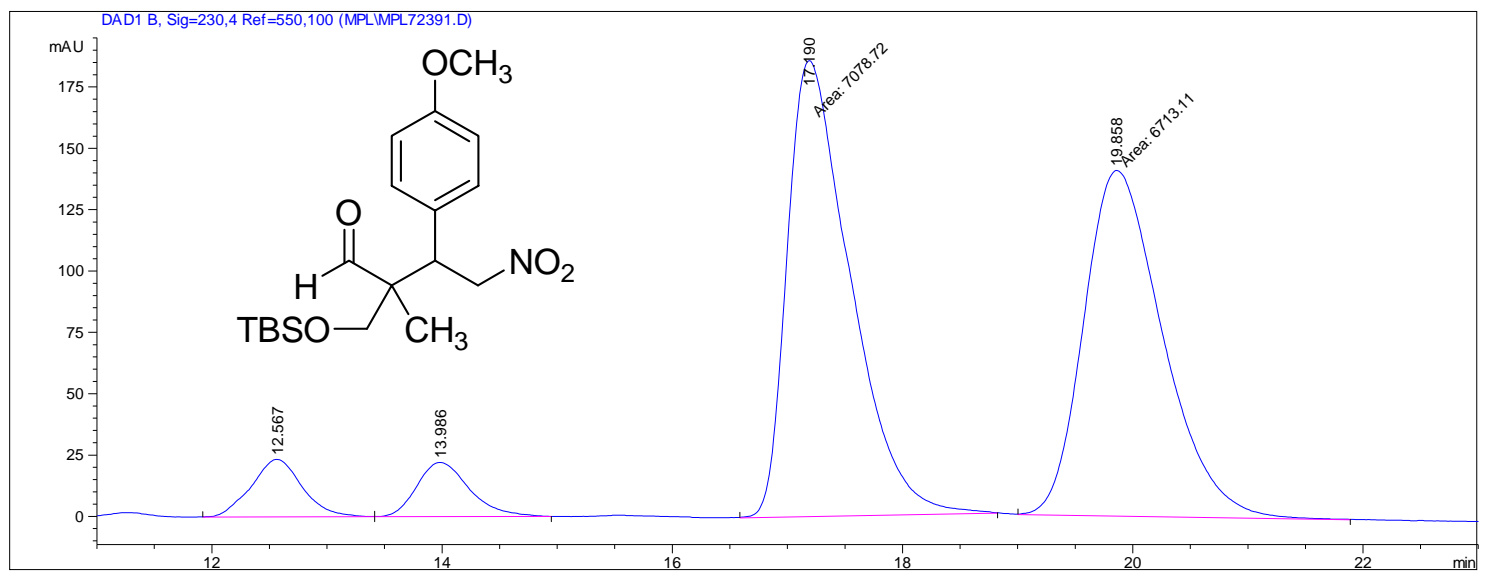

\begin{tabular}{|l|l|l|l|l|l|l|}
\hline $\begin{array}{l}\text { Peak } \\
\#\end{array}$ & $\begin{array}{l}\text { RetTime } \\
{[\mathrm{min}]}\end{array}$ & Type & $\begin{array}{l}\text { Width } \\
{[\mathrm{min}]}\end{array}$ & $\begin{array}{l}\text { Area } \\
{\left[\mathrm{mAU}{ }^{*} \mathrm{~s}\right]}\end{array}$ & $\begin{array}{l}\text { Height } \\
{[\mathrm{mAU}]}\end{array}$ & $\begin{array}{l}\text { Area } \\
{[\%]}\end{array}$ \\
\hline 1 & 12.567 & $\mathrm{VV}$ & 0.4630 & 723.73651 & 23.43773 & 4.7555 \\
\hline 2 & 13.986 & $\mathrm{VV}$ & 0.4589 & 703.28253 & 22.13266 & 4.6211 \\
\hline 3 & 17.190 & $\mathrm{MM}$ & 0.6346 & 7078.71680 & 185.90570 & 46.5128 \\
\hline 4 & 19.858 & $\mathrm{MM}$ & 0.7937 & 6713.10840 & 140.97058 & 44.1105 \\
\hline Totals: & & & & $1.52188 \mathrm{e} 4$ & 372.44667 & \\
\hline
\end{tabular}


Enantioenriched (2S,3R)-2-((tert-butyldimethylsilyloxy)methyl)-3-(4-

methoxyphenyl)-2-methyl-4-nitrobutanal + (2R,3R)-2-((tert-

butyldimethylsilyloxy)methyl)-3-(4-methoxyphenyl)-2-methyl-4-nitrobutanal

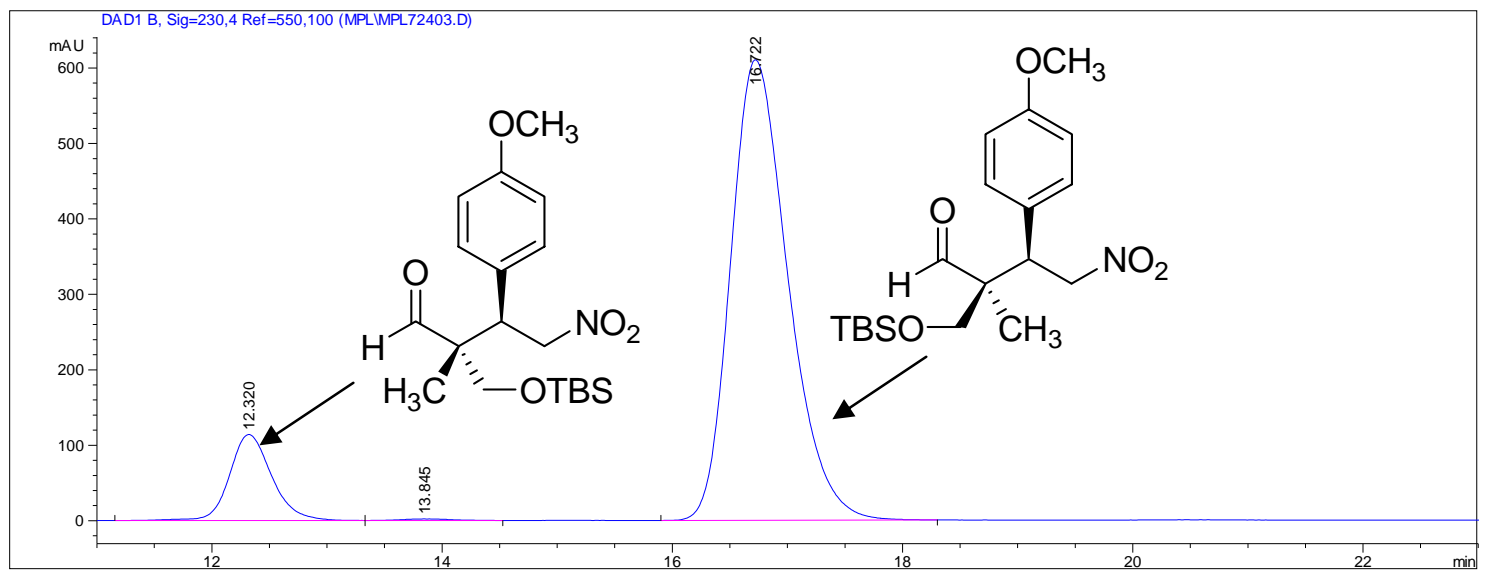

\begin{tabular}{|l|l|l|l|l|l|l|}
\hline $\begin{array}{l}\text { Peak } \\
\#\end{array}$ & $\begin{array}{l}\text { RetTime } \\
{[\mathrm{min}]}\end{array}$ & Type & $\begin{array}{l}\text { Width } \\
{[\mathrm{min}]}\end{array}$ & $\begin{array}{l}\text { Area } \\
{\left[\mathrm{mAU}^{*} \mathrm{~s}\right]}\end{array}$ & $\begin{array}{l}\text { Height } \\
{[\mathrm{mAU}]}\end{array}$ & $\begin{array}{l}\text { Area } \\
{[\%]}\end{array}$ \\
\hline 1 & 12.320 & $\mathrm{VV}$ & 0.3901 & 2920.71484 & 114.09811 & 12.1030 \\
\hline 2 & 13.845 & VV & 0.3947 & 66.05161 & 2.04576 & 0.2737 \\
\hline 3 & 16.722 & PV & 0.5300 & $2.11454 \mathrm{e} 4$ & 610.57416 & 87.6233 \\
\hline Totals: & & & & $2.41322 \mathrm{e} 4$ & 726.71802 & \\
\hline
\end{tabular}

(2S,3R)-2-((tert-butyldimethylsilyloxy)methyl)-2-methyl-4-nitro-3-(thiophen-2-

yl)butanal (14): General procedure, diastereomeric products separable by chromatography on silica (10\% diethyl ether/hexanes), colorless to light yellow oil, $85 \%$ yield $(304.9 \mathrm{mg}), 6.6: 1 \mathrm{dr}$ (determined using the HPLC conditions below and the crude product mixture), $99 \%$ ee (major diastereomer) as determined by HPLC (Chiralcel OD$\mathrm{H}, 1.0 \%$ ethanol/hexanes, $1.0 \mathrm{~mL} / \mathrm{min}, 208 \mathrm{~nm}$; $t_{r}$ (major enantiomer, major diastereomer) $=10.21 \mathrm{~min}, t_{r}($ minor enantiomer, major diastereomer $\left.)=11.75 \mathrm{~min}\right) .[\alpha]^{25} \mathrm{D}=+20.4(\mathrm{c}=$ $0.0101 \mathrm{~g} / 2.0 \mathrm{~mL}$, chloroform). ${ }^{1} \mathrm{H} \mathrm{NMR}\left(400 \mathrm{MHz}, \mathrm{CDCl}_{3}\right): \delta 9.61(1 \mathrm{H}, \mathrm{s}), 7.24(1 \mathrm{H}, \mathrm{dd}$, $\mathrm{J}=1.1,5.1 \mathrm{~Hz}), 6.96(1 \mathrm{H}, \mathrm{dd}, \mathrm{J}=3.5,5.1 \mathrm{~Hz}), 6.95(1 \mathrm{H}, \mathrm{dd}, \mathrm{J}=1.1,3.5 \mathrm{~Hz}), 4.80(1 \mathrm{H}$, $\mathrm{dd}, \mathrm{J}=4.4,13.2 \mathrm{~Hz}), 4.75(1 \mathrm{H}, \mathrm{dd}, \mathrm{J}=10.6,13.2 \mathrm{~Hz}), 4.46(1 \mathrm{H}, \mathrm{dd}, \mathrm{J}=4.4,10.6 \mathrm{~Hz})$, $3.59(1 \mathrm{H}, \mathrm{d}, \mathrm{J}=10.6 \mathrm{~Hz}), 3.45(1 \mathrm{H}, \mathrm{d}, 10.6 \mathrm{~Hz}), 1.11(3 \mathrm{H}, \mathrm{s}), 0.89(9 \mathrm{H}, \mathrm{s}), 0.02(6 \mathrm{H}, \mathrm{s})$. ${ }^{13} \mathrm{C}\left(100 \mathrm{MHz}, \mathrm{CDCl}_{3}\right): \delta 203.4,137.7,127.8,126.8,125.4,77.8,65.8,53.4,40.2,25.7$, 18.1, 13.6, -5.7, -5.8. IR (neat): $2956(\mathrm{~m}), 2930(\mathrm{~m}), 2857(\mathrm{~m}), 2711(\mathrm{w}), 1728(\mathrm{~m})$, $1556(\mathrm{~s}), 1437(\mathrm{~m}), 1378(\mathrm{~m}), 1253(\mathrm{~m}), 1098(\mathrm{~m}), 839(\mathrm{~s}), 779$ (m), 702 (m). HRMS (ESI): expected for $\left[\mathrm{C}_{16} \mathrm{H}_{27} \mathrm{NO}_{4} \mathrm{SSi}+\mathrm{NH}_{4}\right]^{+}: 375.1774$, found: 375.1764 . 
Racemic 2-((tert-butyldimethylsilyloxy)methyl)-2-methyl-4-nitro-3-(thiophen-2yl)butanal

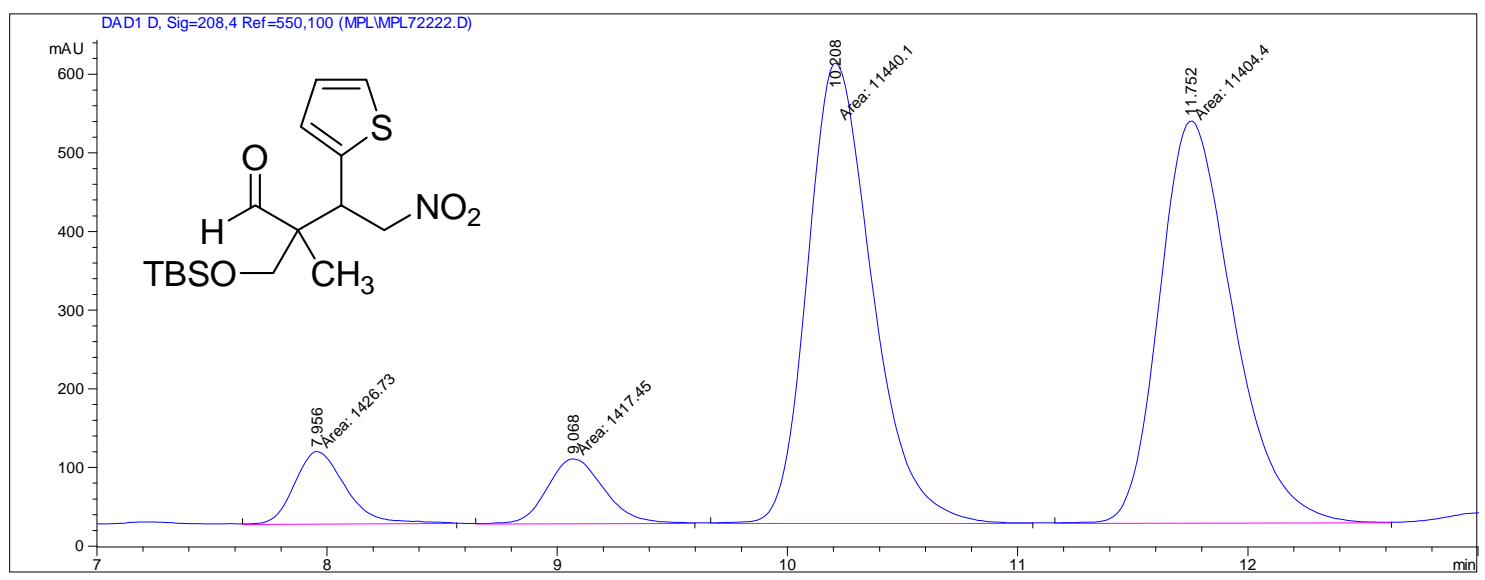

\begin{tabular}{|l|l|l|l|l|l|l|}
\hline $\begin{array}{l}\text { Peak } \\
\#\end{array}$ & $\begin{array}{l}\text { RetTime } \\
{[\mathrm{min}]}\end{array}$ & Type & $\begin{array}{l}\text { Width } \\
{[\mathrm{min}]}\end{array}$ & $\begin{array}{l}\text { Area } \\
{[\mathrm{mAU} \text { s }]}\end{array}$ & $\begin{array}{l}\text { Height } \\
{[\mathrm{mAU}]}\end{array}$ & $\begin{array}{l}\text { Area } \\
{[\%]}\end{array}$ \\
\hline 1 & 7.956 & MM & 0.2574 & 1426.73389 & 92.36478 & 5.5540 \\
\hline 2 & 9.068 & $\mathrm{MM}$ & 0.2859 & 1417.45203 & 82.62922 & 5.5178 \\
\hline 3 & 10.208 & $\mathrm{MM}$ & 0.3254 & $1.14401 \mathrm{e} 4$ & 585.99036 & 44.5336 \\
\hline 4 & 11.752 & $\mathrm{MM}$ & 0.3713 & $1.14044 \mathrm{e} 4$ & 511.94601 & 44.3946 \\
\hline Totals: & & & & $2.56886 \mathrm{e} 4$ & 1272.93037 & \\
\hline
\end{tabular}

Enantioenriched (2S,3R)-2-((tert-butyldimethylsilyloxy)methyl)-2-methyl-4-nitro-3(thiophen-2-yl)butanal

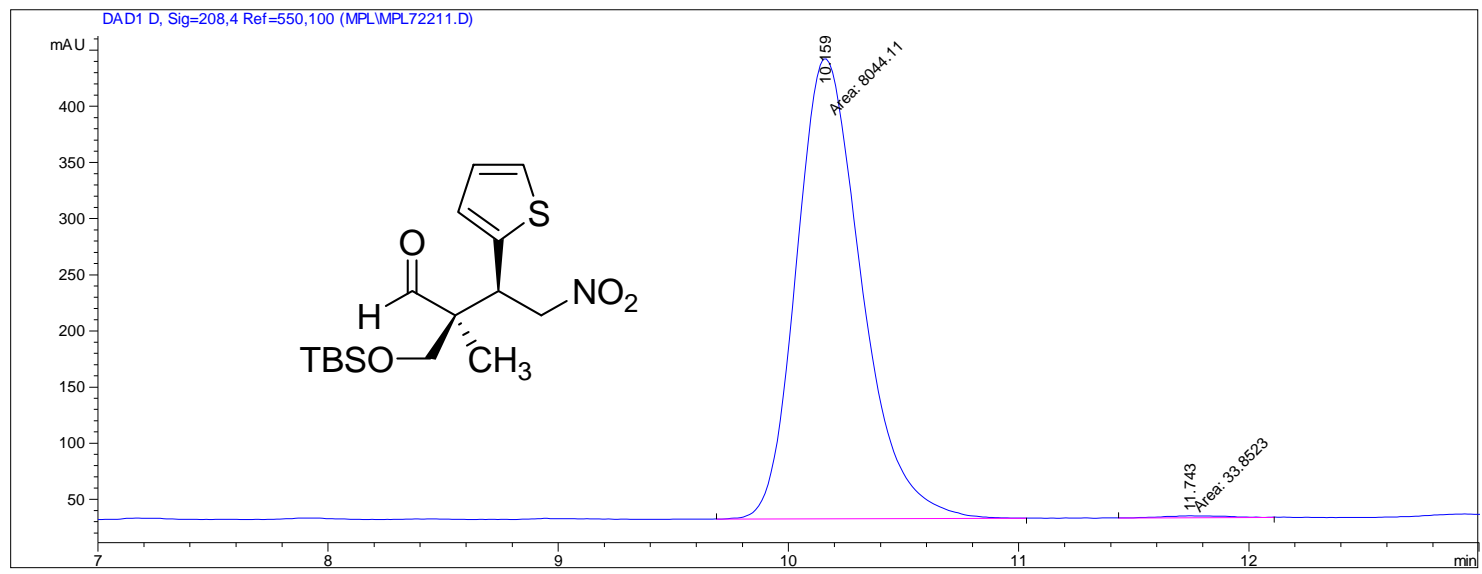

\begin{tabular}{|l|l|l|l|l|l|l|}
\hline $\begin{array}{l}\text { Peak } \\
\#\end{array}$ & $\begin{array}{l}\text { RetTime } \\
{[\mathrm{min}]}\end{array}$ & Type & $\begin{array}{l}\text { Width } \\
{[\mathrm{min}]}\end{array}$ & $\begin{array}{l}\text { Area } \\
{\left[\mathrm{mAU}^{*} \mathrm{~s}\right]}\end{array}$ & $\begin{array}{l}\text { Height } \\
{[\mathrm{mAU}]}\end{array}$ & $\begin{array}{l}\text { Area } \\
{[\%]}\end{array}$ \\
\hline 1 & 10.159 & $\mathrm{MM}$ & 0.3267 & 8044.11377 & 410.32178 & 99.5809 \\
\hline 2 & 11.743 & $\mathrm{MM}$ & 0.3171 & 33.85229 & 1.77910 & 0.4191 \\
\hline Totals: & & & & 8077.96606 & 412.10087 & \\
\hline
\end{tabular}


(2R,3R)-2-((tert-butyldimethylsilyloxy)methyl)-2-methyl-4-nitro-3-(thiophen-2-

yl)butanal: $95 \%$ ee (minor diastereomer) as determined by HPLC (Chiralcel OD-H, $5.0 \%$ isopropanol/hexanes, $1.0 \mathrm{~mL} / \mathrm{min}, 208 \mathrm{~nm}$; $t_{r}$ (major enantiomer, minor diastereomer $)=7.74 \mathrm{~min}, t_{r}($ minor enantiomer, minor diastereomer $\left.)=8.98 \mathrm{~min}\right) .[\alpha]_{\mathrm{D}}^{25}=$ $-2.3\left(\mathrm{c}=0.0173 \mathrm{~g} / 2.0 \mathrm{~mL}\right.$, chloroform). ${ }^{1} \mathrm{H} \mathrm{NMR}\left(400 \mathrm{MHz}, \mathrm{CDCl}_{3}\right): \delta 9.61(1 \mathrm{H}, \mathrm{s})$, $7.25(1 \mathrm{H}, \mathrm{dd}, \mathrm{J}=1.1,5.1 \mathrm{~Hz}), 6.96(1 \mathrm{H}, \mathrm{dd}, \mathrm{J}=3.5,5.1 \mathrm{~Hz}), 6.93(1 \mathrm{H}, \mathrm{dd}, \mathrm{J}=1.1,3.5$ $\mathrm{Hz}), 4.89(1 \mathrm{H}, \mathrm{dd}, \mathrm{J}=11.3,13.5 \mathrm{~Hz}), 4.80(1 \mathrm{H}, \mathrm{dd}, \mathrm{J}=3.7,13.5 \mathrm{~Hz}), 4.40(1 \mathrm{H}, \mathrm{dd}, \mathrm{J}=$ 3.7, $11.3 \mathrm{~Hz}), 3.84(1 \mathrm{H}, \mathrm{d}, \mathrm{J}=11.0 \mathrm{~Hz}), 3.79(1 \mathrm{H}, \mathrm{d}, \mathrm{J}=11.0 \mathrm{~Hz}), 0.92(9 \mathrm{H}, \mathrm{s}), 0.91(3 \mathrm{H}$, s), $0.14(3 \mathrm{H}, \mathrm{s}), 0.10(3 \mathrm{H}, \mathrm{s}) .{ }^{13} \mathrm{C}\left(100 \mathrm{MHz}, \mathrm{CDCl}_{3}\right): \delta 202.9,138.2,127.9,126.9,125.5$, 78.5, 64.8, 53.6, 42.2, 25.8, 18.1, 16.9, -5.6, -5.7. IR (neat): 2956 (s), 2930 (s), 2858 (s), 2715 (m), 1728 (s), 1555 (s), 1472 (m), 1378 (m), 1254 (m), 1094 (s), 838 (s), 780 (s), 702 (s). HRMS (ESI): expected for $\left[\mathrm{C}_{16} \mathrm{H}_{27} \mathrm{NO}_{4} \mathrm{SSi}^{+} \mathrm{NH}_{4}\right]^{+}: 375.1774$, found: 375.1769 .

Enantioenriched (2R,3R)-2-((tert-butyldimethylsilyloxy)methyl)-2-methyl-4-nitro-3(thiophen-2-yl)butanal

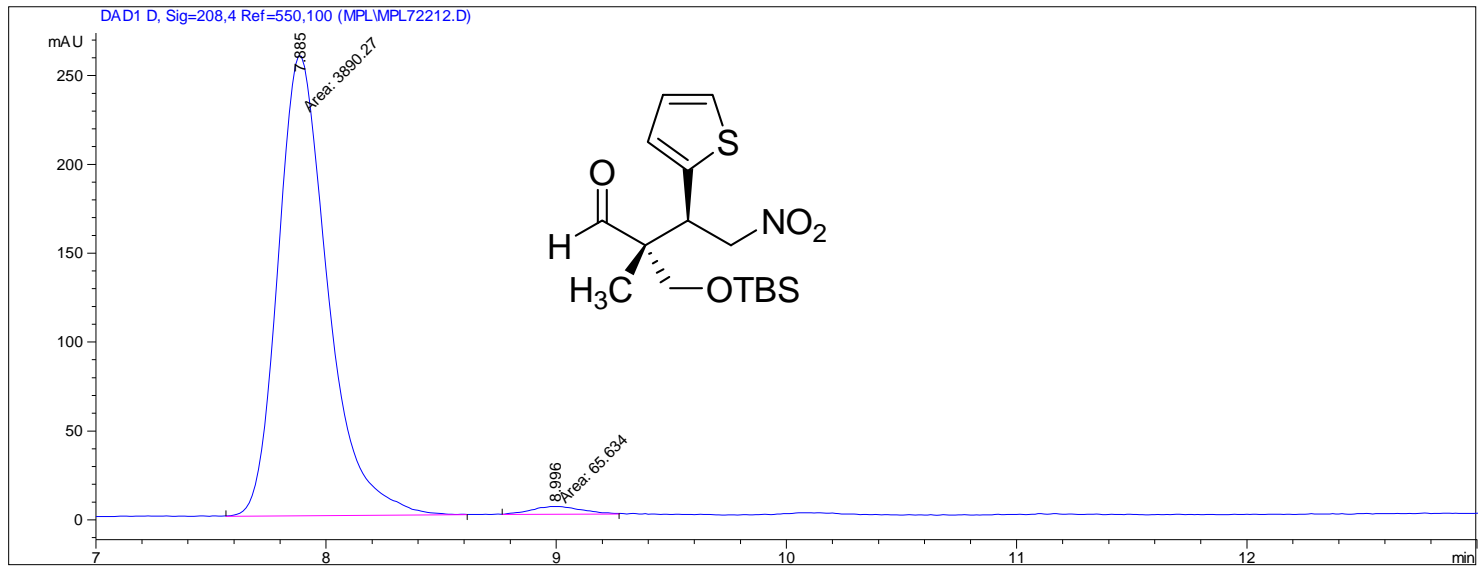

\begin{tabular}{|l|l|l|l|l|l|l|}
\hline $\begin{array}{l}\text { Peak } \\
\#\end{array}$ & $\begin{array}{l}\text { RetTime } \\
{[\mathrm{min}]}\end{array}$ & Type & $\begin{array}{l}\text { Width } \\
{[\mathrm{min}]}\end{array}$ & $\begin{array}{l}\text { Area } \\
{\left[\mathrm{mAU}{ }^{*}\right]}\end{array}$ & $\begin{array}{l}\text { Height } \\
{[\mathrm{mAU}]}\end{array}$ & $\begin{array}{l}\text { Area } \\
{[\%]}\end{array}$ \\
\hline 1 & 7.885 & $\mathrm{MM}$ & 0.2498 & 3890.26636 & 259.57452 & 98.3409 \\
\hline 2 & 8.996 & $\mathrm{MM}$ & 0.2531 & 65.63396 & 4.32216 & 1.6591 \\
\hline Totals: & & & & 3955.90032 & 263.89668 & \\
\hline
\end{tabular}

(2S,3R)-2-((tert-butyldimethylsilyloxy)methyl)-2-methyl-4-nitro-3-(furan-2yl)butanal + (2R,3R)-2-((tert-butyldimethylsilyloxy)methyl)-2-methyl-4-nitro-3(furan-2-yl)butanal (15): General procedure, chromatography on silica (10\% diethyl ether/hexanes) diastereomers could not be separated, colorless to light yellow oil, 79\% yield (271.7 mg), 5.4:1 dr, 99\% ee (major diastereomer), 95\% ee (minor diastereomer) as determined by HPLC (Chiralcel OD-H, 1.0\% isopropanol/hexanes, $1.0 \mathrm{~mL} / \mathrm{min}, 208 \mathrm{~nm}$; $t_{r}($ major enantiomer, minor diastereomer $)=8.73 \mathrm{~min}, t_{r}($ minor enantiomer, minor 
diastereomer $)=9.82 \mathrm{~min}, t_{r}($ major enantiomer, major diastereomer $)=10.55 \mathrm{~min}$, $t_{r}($ minor enantiomer, major diastereomer $\left.)=13.21 \mathrm{~min}\right) \cdot[\alpha]_{\mathrm{D}}^{25}=+3.0(\mathrm{c}=0.0209 \mathrm{~g} / 2.0$ $\mathrm{mL}$, chloroform). ${ }^{1} \mathrm{H}$ NMR (400 $\mathrm{MHz}, \mathrm{CDCl}_{3}$ ): Signals corresponding to the major diastereomer $\delta 9.58(1 \mathrm{H}, \mathrm{s}), 7.35(1 \mathrm{H}, \mathrm{dd}, \mathrm{J}=0.7,1.8 \mathrm{~Hz}), 6.30(1 \mathrm{H}, \mathrm{dd}, \mathrm{J}=1.8,3.3$ $\mathrm{Hz}), 6.22(1 \mathrm{H}, \mathrm{dd}, \mathrm{J}=0.7,1.8 \mathrm{~Hz}), 4.80(1 \mathrm{H}, \mathrm{dd}, \mathrm{J}=11.3,13.2 \mathrm{~Hz}), 4.68(1 \mathrm{H}, \mathrm{dd}, \mathrm{J}=$ $3.7,13.2 \mathrm{~Hz}), 4.21(1 \mathrm{H}, \mathrm{dd}, \mathrm{J}=3.7,11.3 \mathrm{~Hz}), 3.54(1 \mathrm{H}, \mathrm{d}, \mathrm{J}=10.6 \mathrm{~Hz}), 3.42(1 \mathrm{H}, \mathrm{d}, \mathrm{J}=$ $10.6 \mathrm{~Hz}), 1.09(3 \mathrm{H}, \mathrm{s}), 0.87(9 \mathrm{H}, \mathrm{s}), 0.00(6 \mathrm{H}, \mathrm{s})$. Signals corresponding to the minor diastereomer $\delta 9.57(1 \mathrm{H}, \mathrm{s}), 7.35(1 \mathrm{H}, \mathrm{dd}, \mathrm{J}=0.7,1.8 \mathrm{~Hz}), 6.30(1 \mathrm{H}, \mathrm{dd}, \mathrm{J}=1.8,3.3 \mathrm{~Hz})$, $6.20(1 \mathrm{H}, \mathrm{dd}, \mathrm{J}=0.7,1.8 \mathrm{~Hz}), 4.89(1 \mathrm{H}, \mathrm{dd}, \mathrm{J}=11.3,13.2 \mathrm{~Hz}), 4.73(1 \mathrm{H}, \mathrm{dd}, \mathrm{J}=3.7$, $13.2 \mathrm{~Hz}), 4.18(1 \mathrm{H}, \mathrm{dd}, \mathrm{J}=3.7,11.3 \mathrm{~Hz}), 3.78(1 \mathrm{H}, \mathrm{d}, \mathrm{J}=10.6 \mathrm{~Hz}), 3.75(1 \mathrm{H}, \mathrm{d}, \mathrm{J}=10.6$ $\mathrm{Hz}), 0.90(9 \mathrm{H}, \mathrm{s}), 0.89(3 \mathrm{H}, \mathrm{s}), 0.10(3 \mathrm{H}, \mathrm{s}), 0.07(3 \mathrm{H}, \mathrm{s}) .{ }^{13} \mathrm{C}\left(100 \mathrm{MHz}, \mathrm{CDCl}_{3}\right)$ : Signals corresponding to the major diastereomer $\delta 203.3,149.7,142.6,110.4,109.8,74.9,53.2$, $38.5,25.7,18.0,13.9,-5.8,-5.9$. Signals corresponding to the minor diastereomer $\delta$ 202.8, 150.1, 142.7, 110.4, 109.6, 75.3, 65.2, 53.4, 40.1, 25.7, 18.1, 16.3, -5.7, -5.8. IR (neat): 2958 (s), 2931 (s), 2886 (m), 2858 (s), 2713 (m), 1733 (s), 1557 (s), 1472 (m), 1376 (m), 1254 (m), 1101 (s), 1015 (m), 840 (s), 779 (s), 737 (m). HRMS (ESI): expected for $\left[\mathrm{C}_{16} \mathrm{H}_{27} \mathrm{NO}_{5} \mathrm{Si}+\mathrm{NH}_{4}\right]^{+}: 359.2002$, found: 359.1987 .

\section{Racemic yl)butanal \\ 2-((tert-butyldimethylsilyloxy)methyl)-2-methyl-4-nitro-3-(furan-2-}

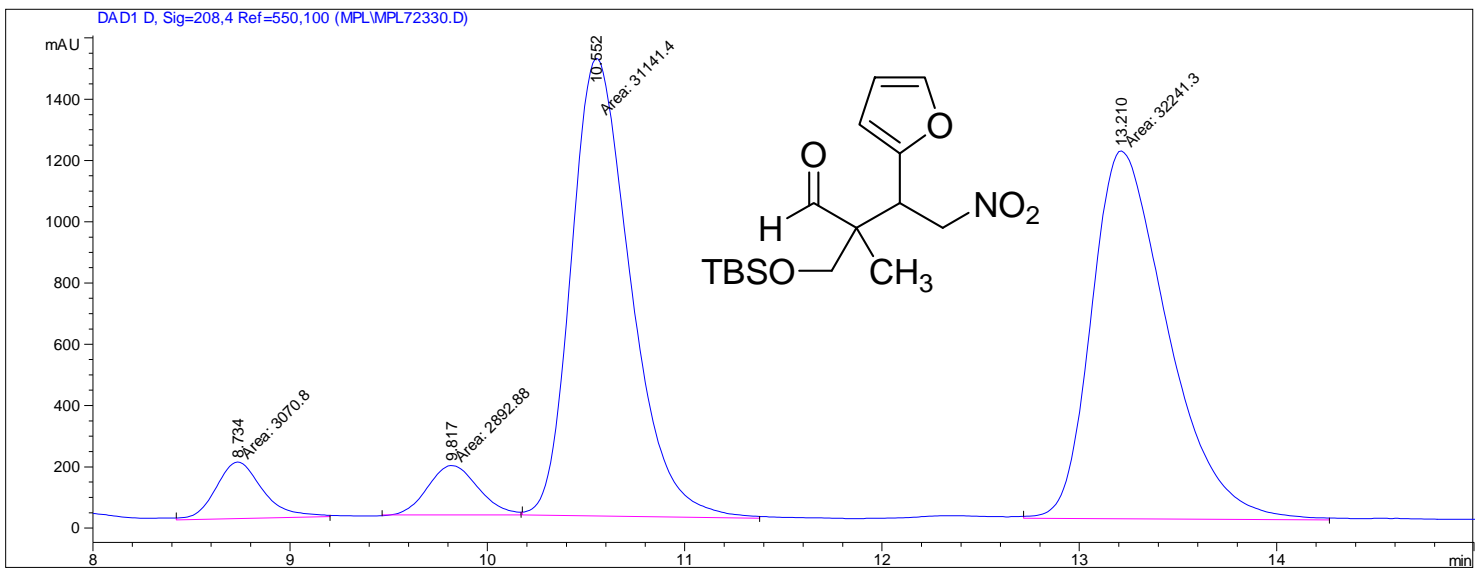

\begin{tabular}{|l|l|l|l|l|l|l|}
\hline $\begin{array}{l}\text { Peak } \\
\#\end{array}$ & $\begin{array}{l}\text { RetTime } \\
{[\mathrm{min}]}\end{array}$ & Type & $\begin{array}{l}\text { Width } \\
{[\mathrm{min}]}\end{array}$ & $\begin{array}{l}\text { Area } \\
{[\mathrm{mAU} * \mathrm{~s}]}\end{array}$ & $\begin{array}{l}\text { Height } \\
{[\mathrm{mAU}]}\end{array}$ & $\begin{array}{l}\text { Area } \\
{[\%]}\end{array}$ \\
\hline 1 & 8.734 & $\mathrm{MM}$ & 0.2887 & 3220.02832 & 185.88496 & 4.5858 \\
\hline 2 & 9.817 & $\mathrm{MM}$ & 0.3198 & 3283.60132 & 171.10840 & 4.6763 \\
\hline 3 & 10.552 & $\mathrm{MM}$ & 0.3522 & $3.18460 \mathrm{e} 4$ & 1507.09766 & 45.3534 \\
\hline 4 & 13.210 & $\mathrm{MM}$ & 0.4432 & $3.18679 \mathrm{e} 4$ & 1198.31396 & 45.3845 \\
\hline Totals: & & & & $7.02176 \mathrm{e} 4$ & 3062.40498 & \\
\hline
\end{tabular}


Enantioenriched (2S,3R)-2-((tert-butyldimethylsilyloxy)methyl)-2-methyl-4-nitro-3(furan-2-yl)butanal $+(2 R, 3 R)-2-(($ tert-butyldimethylsilyloxy)methyl)-2-methyl-4nitro-3-(furan-2-yl)butanal

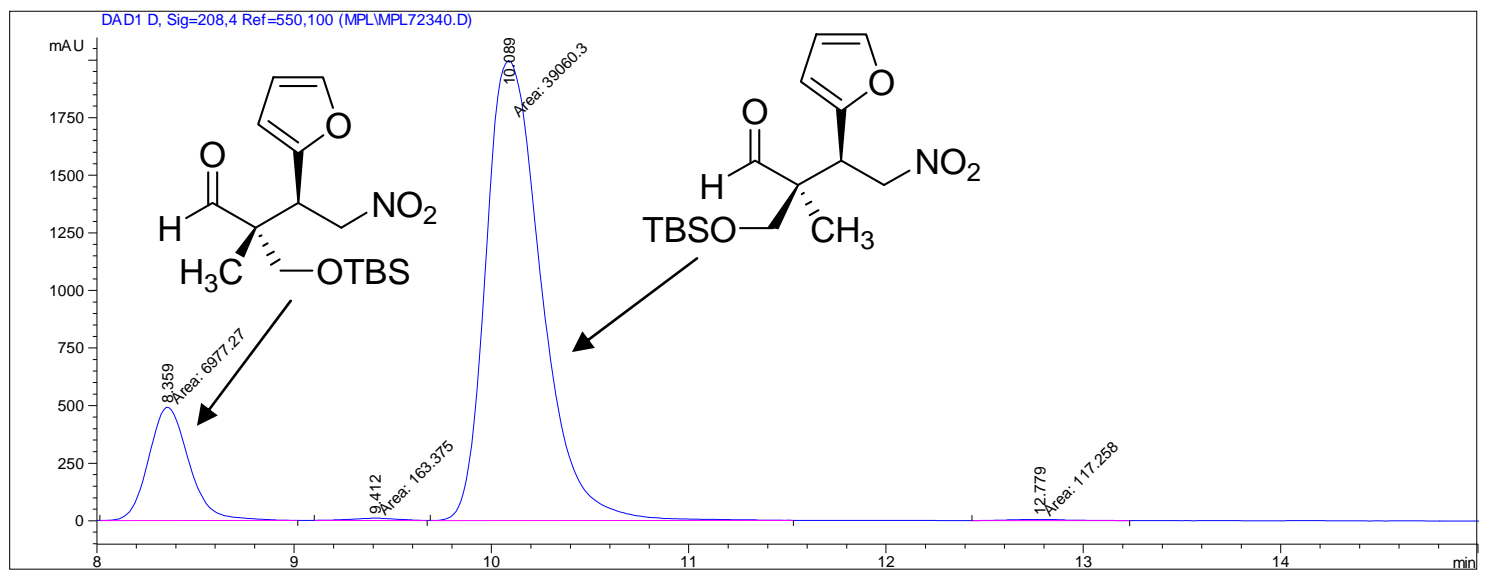

\begin{tabular}{|l|l|l|l|l|l|l|}
\hline $\begin{array}{l}\text { Peak } \\
\#\end{array}$ & $\begin{array}{l}\text { RetTime } \\
{[\mathrm{min}]}\end{array}$ & Type & $\begin{array}{l}\text { Width } \\
{[\mathrm{min}]}\end{array}$ & $\begin{array}{l}\text { Area } \\
{[\mathrm{mAU} * \mathrm{~s}]}\end{array}$ & $\begin{array}{l}\text { Height } \\
{[\mathrm{mAU}]}\end{array}$ & $\begin{array}{l}\text { Area } \\
{[\%]}\end{array}$ \\
\hline 1 & 8.359 & $\mathrm{MM}$ & 0.2374 & 7019.18848 & 492.82800 & 15.1191 \\
\hline 2 & 9.412 & $\mathrm{MM}$ & 0.2874 & 173.29816 & 10.04999 & 0.3733 \\
\hline 3 & 10.089 & $\mathrm{MM}$ & 0.3259 & $3.90897 \mathrm{e} 4$ & 1999.21619 & 84.1981 \\
\hline 4 & 12.779 & $\mathrm{MM}$ & 0.4413 & 143.67551 & 5.42637 & 0.3095 \\
\hline Totals: & & & & $4.64258 \mathrm{e} 4$ & 2507.52056 & \\
\hline
\end{tabular}

(2S,3R)-2-((tert-butyldimethylsilyloxy)methyl)-2-methyl-4-nitro-3-(pyridin-3yl)butanal + (2R,3R)-2-((tert-butyldimethylsilyloxy)methyl)-2-methyl-4-nitro-3(pyridin-3-yl)butanal (16): Under a positive pressure of nitrogen at room temperature, thiourea catalyst $1(75.3 \mathrm{mg}, 0.20 \mathrm{mmol}, 20 \mathrm{~mol} \%)$ was loaded into an oven-dried $25 \mathrm{~mL}$ round-bottomed flask, equipped with a magnetic stir bar, rubber septum, and nitrogen inlet. The catalyst was dissolved in dichloromethane $(6.7 \mathrm{~mL})$. Water $(90.1 \mu \mathrm{L}, 5.0$ mmol, 5.0 equiv.) and 3-(tert-butyldimethylsilyloxy)-2-methylpropanal (404.8 $\mu \mathrm{g}, 2.0$ mmol, 2.0 equiv.) were subsequently added via syringe. The resulting clear colorless solution was stirred for approximately two minutes. Solid 3-(2-nitroethenyl)pyridine (150.1 $\mathrm{mg}, 1.0 \mathrm{mmol}, 1.0$ equiv.) was added in one portion resulting in a dark yellow solution. The rubber septum was quickly replaced with a yellow polyethylene stopper (to prevent dichloromethane evaporation) and the reaction mixture was stirred for 24 hours at room temperature. Aqueous hydrochloric acid solution $(1 \mathrm{M}, 7 \mathrm{~mL})$ was added to the reaction flask and the resulting biphasic mixture was stirred vigorously for 5 minutes at room temperature. The acidic solution was neutralized by the portion wise addition of solid sodium bicarbonate. The biphasic mixture was transferred to a separatory funnel and additional portions of dichloromethane $(30 \mathrm{~mL})$ and saturated aqueous sodium bicarbonate $(30 \mathrm{~mL})$ were added. The phases were separated and the aqueous layer was washed with dichloromethane $(30 \mathrm{~mL})$. The organic layers were combined and washed with saturated aqueous sodium chloride solution $(30 \mathrm{~mL})$, dried over anhydrous sodium 
sulfate, filtered, and concentrated in vacuo. Chromatography on silica $(1.0 \%$ methanol/dichloromethane) diastereomers could not be separated, colorless to light yellow oil, 94\% yield (332.3 mg), 5.6:1 dr as determined by SFC (Chiralcel OD-H, 2.0\% methanol $/ \mathrm{CO}_{2}, 2.0 \mathrm{~mL} / \mathrm{min}, 208 \mathrm{~nm}, 30{ }^{\circ} \mathrm{C} ; t_{r}$ (minor enantiomer, minor diastereomer $)=$ $8.39 \mathrm{~min}, t_{r}($ major enantiomer, minor diastereomer $)=10.49 \mathrm{~min}, t_{r}$ (major enantiomer, major diastereomer $)=13.11 \mathrm{~min}, t_{r}($ minor enantiomer, major diastereomer $\left.)=14.03 \mathrm{~min}\right)$, 99\% ee (major diastereomer) as determined by HPLC (Chiralcel OD-H 2.5\% ethanol/hexanes, $1.0 \mathrm{~mL} / \mathrm{min}, 208 \mathrm{~nm}$; $t_{r}$ (minor enantiomer, major diastereomer) $=30.27$ $\min , t_{r}$ (minor enantiomer, major diastereomer) $=34.50 \mathrm{~min}$ ), $96 \%$ ee (minor diastereomer) as determined by SFC (Chiralcel OD-H, $2.0 \%$ methanol $/ \mathrm{CO}_{2}, 2.0 \mathrm{~mL} / \mathrm{min}$, $208 \mathrm{~nm}, 30^{\circ} \mathrm{C} ; t_{r}$ (minor enantiomer, minor diastereomer) $=8.39 \mathrm{~min}, t_{r}$ (major enantiomer, minor diastereomer $)=10.49 \mathrm{~min}) .[\alpha]_{\mathrm{D}}^{25}=+7.3(\mathrm{c}=0.0200 \mathrm{~g} / 2.0 \mathrm{~mL}$, chloroform). ${ }^{1} \mathrm{H}$ NMR $\left(400 \mathrm{MHz}, \mathrm{CDCl}_{3}\right)$ : Signals corresponding to the major diastereomer $\delta 9.54(1 \mathrm{H}, \mathrm{s}), 8.53(2 \mathrm{H}, \mathrm{m}), 7.62(1 \mathrm{H}, \mathrm{dt}, \mathrm{J}=1.8,8.1 \mathrm{~Hz}), 7.25(1 \mathrm{H}, \mathrm{ddd}, \mathrm{J}$ $=0.7,4.0,8.1 \mathrm{~Hz}), 4.88(2 \mathrm{H}, \mathrm{d}, \mathrm{J}=7.7 \mathrm{~Hz}), 4.16(1 \mathrm{H}, \mathrm{t}, \mathrm{J}=7.7 \mathrm{~Hz}), 3.56(1 \mathrm{H}, \mathrm{d}, \mathrm{J}=10.6$ $\mathrm{Hz}), 3.25(1 \mathrm{H}, \mathrm{d}, \mathrm{J}=10.6 \mathrm{~Hz}), 1.02(3 \mathrm{H}, \mathrm{s}), 0.88(9 \mathrm{H}, \mathrm{s}), 0.00(6 \mathrm{H}, \mathrm{s})$. Signals corresponding to the minor diastereomer $\delta 9.56(1 \mathrm{H}, \mathrm{s}), 8.53(1 \mathrm{H}, \mathrm{m}), 8.48(1 \mathrm{H}, \mathrm{dd}, \mathrm{J}=$ $1.5,4.8 \mathrm{~Hz}), 7.59(1 \mathrm{H}, \mathrm{dt}, \mathrm{J}=1.8,8.1 \mathrm{~Hz}), 7.27(1 \mathrm{H}, \mathrm{ddd}, \mathrm{J}=0.7,4.0,8.1 \mathrm{~Hz}), 5.06(1 \mathrm{H}$, $\mathrm{dd}, \mathrm{J}=11.7,13.9 \mathrm{~Hz}), 4.85(1 \mathrm{H}, \mathrm{dd}, \mathrm{J}=3.3,13.9 \mathrm{~Hz}), 4.02(1 \mathrm{H}, \mathrm{dd}, \mathrm{J}=3.3,11.7 \mathrm{~Hz})$, $3.77(1 \mathrm{H}, \mathrm{d}, \mathrm{J}=11.7 \mathrm{~Hz}), 3.58(1 \mathrm{H}, \mathrm{d}, \mathrm{J}=11.7 \mathrm{~Hz}), 0.92(9 \mathrm{H}, \mathrm{s}), 0.80(3 \mathrm{H}, \mathrm{s}), 0.12(3 \mathrm{H}$, s), $0.09(3 \mathrm{H}, \mathrm{s}) .{ }^{13} \mathrm{C}\left(100 \mathrm{MHz}, \mathrm{CDCl}_{3}\right)$ : Signals corresponding to the major diastereomer $\delta$ 202.9, 150.6, 149.4, 136.4, 131.4, 123.4, 75.9, 65.6, 53.4, 42.0, 25.7, 18.1, 13.5, -5.7, 5.9. Signals corresponding to the minor diastereomer $\delta 202.5,150.6,149.4,136.5,131.6$, 123.5, 76.4, 64.4, 53.5, 44.3, 25.8, 18.0, 16.9, -5.7, -5.9. IR (neat): 3034 (m), 2955 (s), 2931 (s), 2713 (m), 1728 (s), 1556 (s), 1471 (m), 1429 (m), 1377 (s), 1255 (s), 1097 (s), 839 (s), 780 (s), 714 (s). HRMS (ESI): expected for $\left[\mathrm{C}_{17} \mathrm{H}_{28} \mathrm{~N}_{2} \mathrm{O}_{4} \mathrm{Si}+\mathrm{H}\right]^{+}: 353.1896$, found: 353.1900 . 
Racemic 2-((tert-butyldimethylsilyloxy)methyl)-2-methyl-4-nitro-3-(pyridin-3yl)butanal (minor diastereomer)

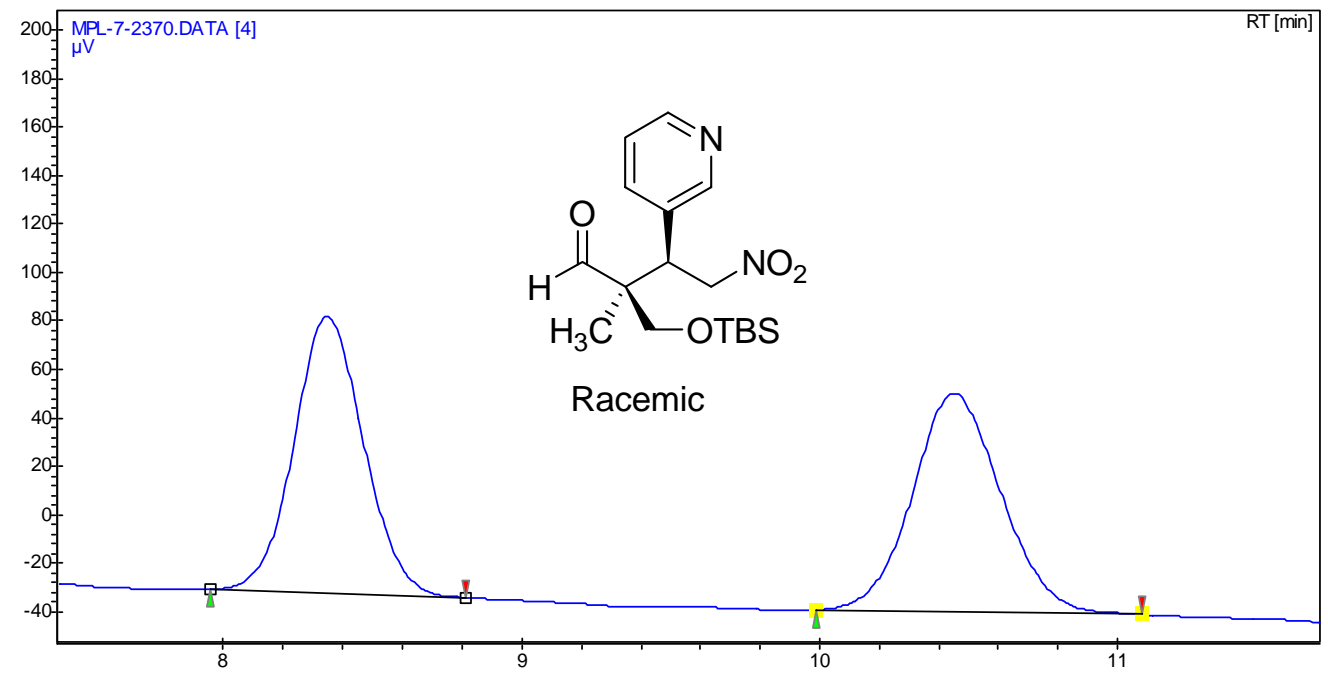

\begin{tabular}{|l|l|l|l|l|}
\hline$\#$ & Time $[$ Min $]$ & Height $[\mu \mathrm{V}]$ & Area $[\mu \mathrm{V}$. Min $]$ & Area [\%] \\
\hline 1 & 8.35 & 114.2 & 31.0 & 49.885 \\
\hline 2 & 10.45 & 90.2 & 31.1 & 50.115 \\
\hline & & & & \\
\hline Total & & 204.4 & 62.1 & 100.000 \\
\hline
\end{tabular}

Enantioenriched (2R,3R)-2-((tert-butyldimethylsilyloxy)methyl)-2-methyl-4-nitro-3(pyridin-3-yl)butanal

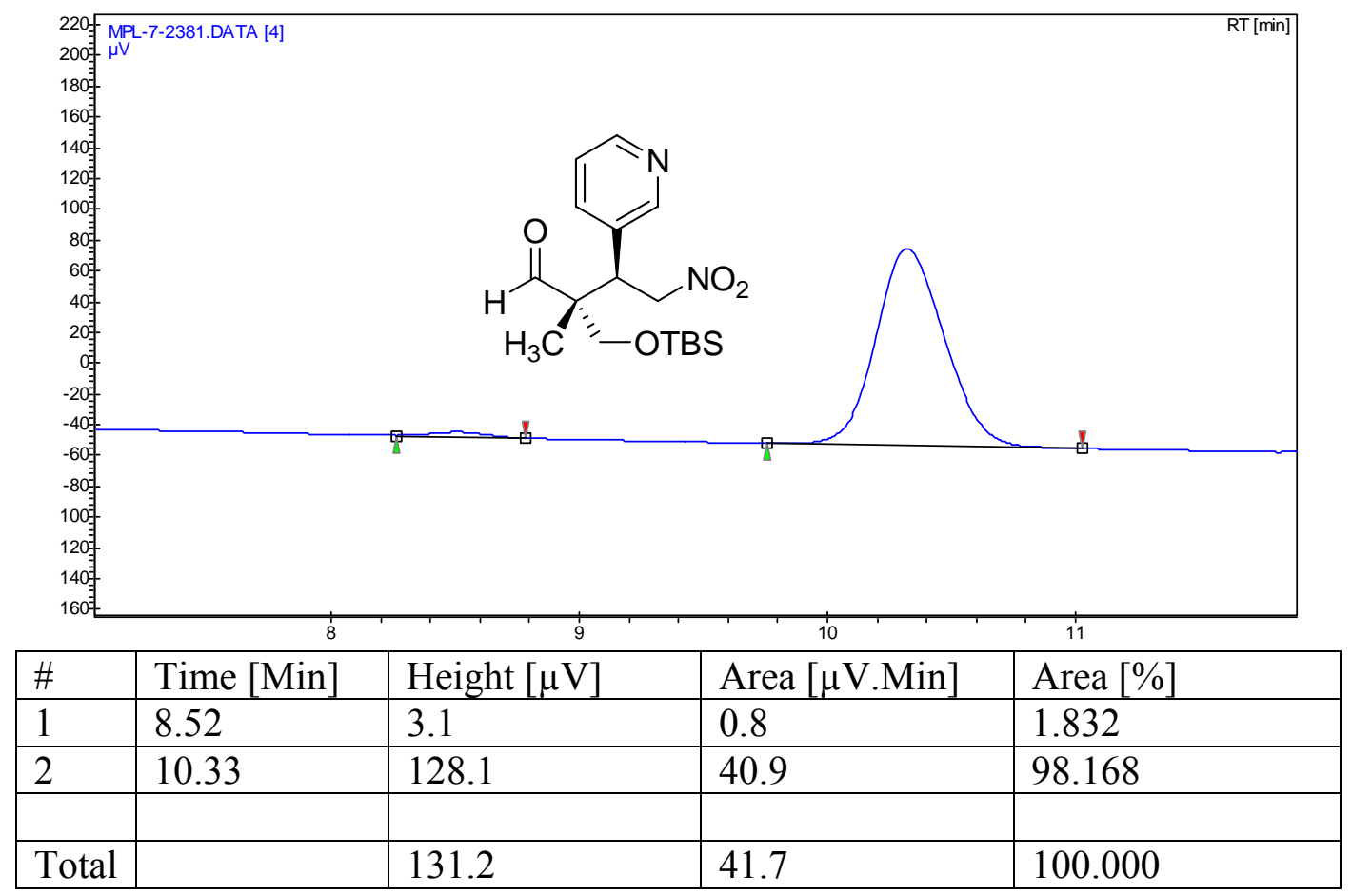


Racemic 2-((tert-butyldimethylsilyloxy)methyl)-2-methyl-4-nitro-3-(pyridin-3yl)butanal (major diastereomer)

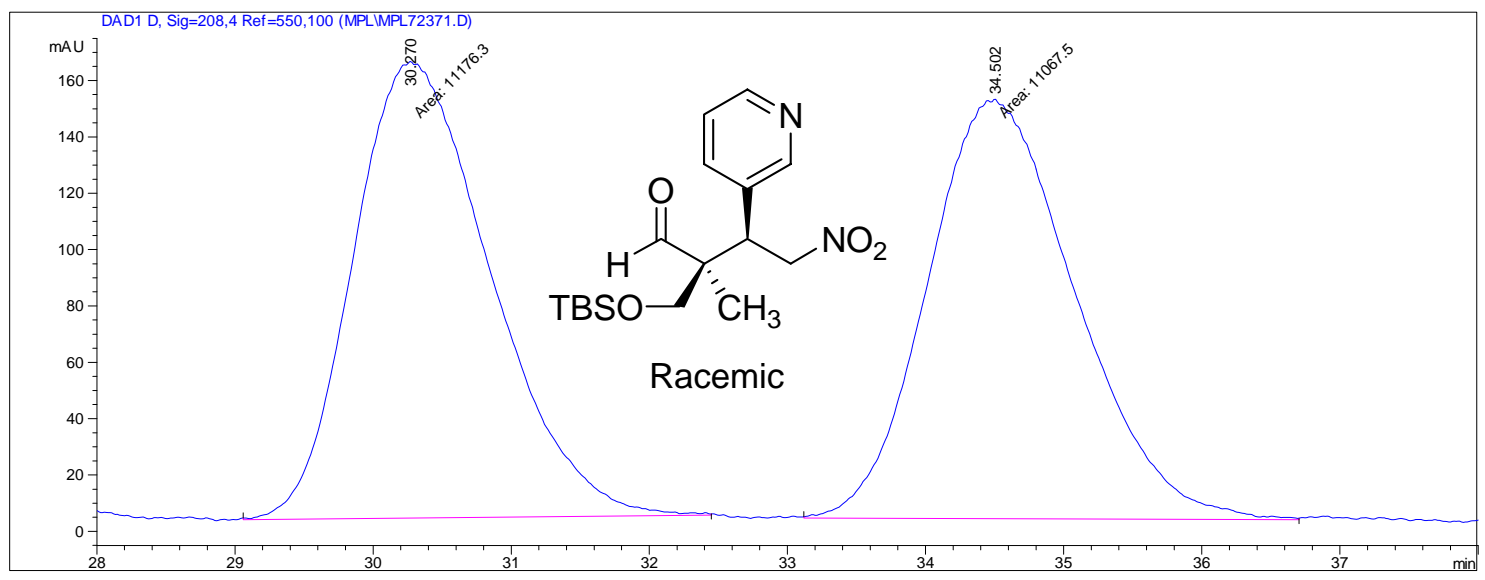

\begin{tabular}{|l|l|l|l|l|l|l|}
\hline $\begin{array}{l}\text { Peak } \\
\#\end{array}$ & $\begin{array}{l}\text { RetTime } \\
{[\mathrm{min}]}\end{array}$ & Type & $\begin{array}{l}\text { Width } \\
{[\mathrm{min}]}\end{array}$ & $\begin{array}{l}\text { Area } \\
{\left[\mathrm{mAU}^{*} \mathrm{~s}\right]}\end{array}$ & $\begin{array}{l}\text { Height } \\
{[\mathrm{mAU}]}\end{array}$ & $\begin{array}{l}\text { Area } \\
{[\%]}\end{array}$ \\
\hline 1 & 30.270 & $\mathrm{MM}$ & 1.1486 & $1.11763 \mathrm{e} 4$ & 162.17905 & 50.2446 \\
\hline 2 & 34.502 & $\mathrm{MM}$ & 1.2375 & $1.10675 \mathrm{e} 4$ & 149.05533 & 49.7554 \\
\hline Totals: & & & & $2.22438 \mathrm{e} 4$ & 311.23438 & \\
\hline
\end{tabular}

Enantioenriched (2S,3R)-2-((tert-butyldimethylsilyloxy)methyl)-2-methyl-4-nitro-3(pyridin-3-yl)butanal

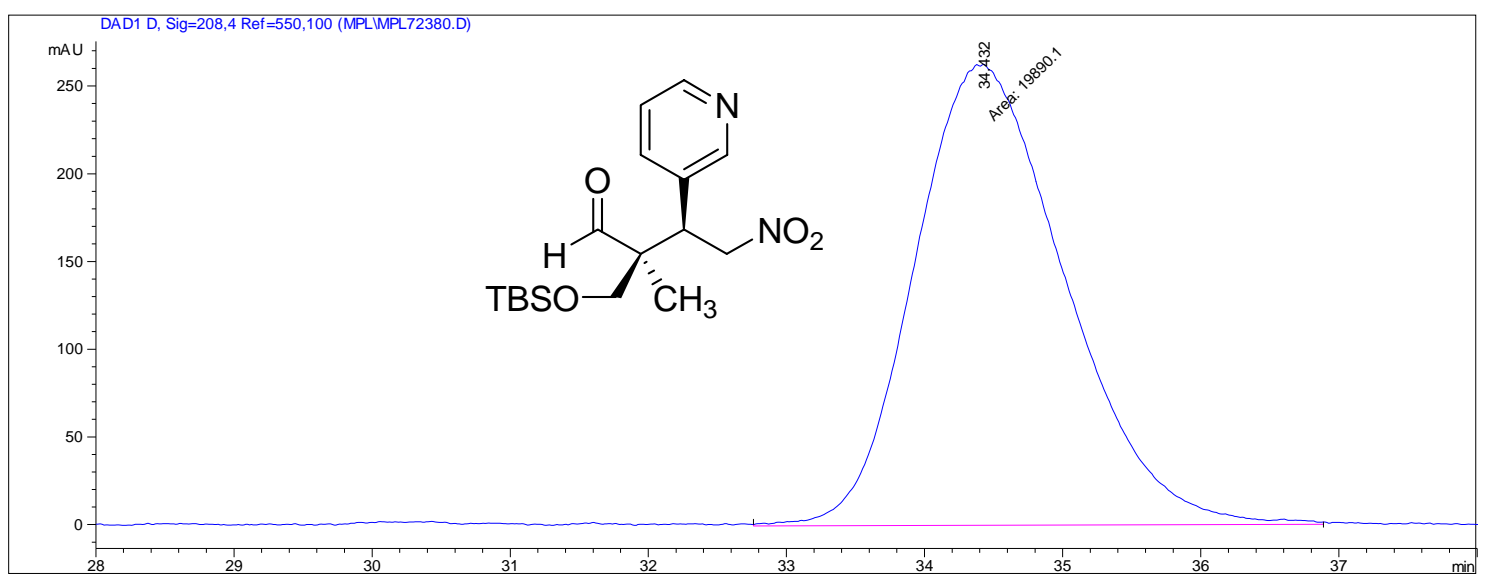

\begin{tabular}{|l|l|l|l|l|l|l|}
\hline $\begin{array}{l}\text { Peak } \\
\#\end{array}$ & $\begin{array}{l}\text { RetTime } \\
{[\mathrm{min}]}\end{array}$ & Type & $\begin{array}{l}\text { Width } \\
{[\mathrm{min}]}\end{array}$ & $\begin{array}{l}\text { Area } \\
{\left[\mathrm{mAU}^{*} \mathrm{~s}\right]}\end{array}$ & $\begin{array}{l}\text { Height } \\
{[\mathrm{mAU}]}\end{array}$ & $\begin{array}{l}\text { Area } \\
{[\%]}\end{array}$ \\
\hline 1 & 34.432 & $\mathrm{MM}$ & 1.2618 & $1.98901 \mathrm{e} 4$ & 262.72925 & 100.0000 \\
\hline Totals: & & & & $1.98901 \mathrm{e} 4$ & 262.72925 & \\
\hline
\end{tabular}


(2R,3S)-2-methyl-4-nitro-2-phenoxy-3-phenylbutanal + (2S,3S)-2-methyl-4-nitro-2phenoxy-3-phenylbutanal (17): General procedure, chromatography on silica $(15 \%$ diethyl ether/hexanes) diastereomers could not be separated, colorless to light yellow oil, $78 \%$ yield $(233.5 \mathrm{mg}$ ), 10.4:1 dr, 92\% ee (minor diastereomer), $94 \%$ ee (major diastereomer) as determined by HPLC $((R, R)$-Whelk-01, $5.0 \%$ isopropanol/hexanes, 1.0 $\mathrm{mL} / \mathrm{min}, 208 \mathrm{~nm}$; $t_{r}$ (major enantiomer, minor diastereomer $)=16.66 \mathrm{~min}, t_{r}($ minor enantiomer, minor diastereomer $)=18.16 \mathrm{~min}, t_{r}($ minor enantiomer, major diastereomer $)$ $=22.21 \mathrm{~min}, t_{r}($ major enantiomer, major diastereomer $\left.)=24.21 \mathrm{~min}\right) .[\alpha]_{\mathrm{D}}^{25}=-61.0(\mathrm{c}=$ $0.0212 \mathrm{~g} / 2.0 \mathrm{~mL}$, chloroform). ${ }^{1} \mathrm{H}$ NMR $\left(400 \mathrm{MHz}, \mathrm{CDCl}_{3}\right)$ : Signals corresponding to the major diastereomer $\delta 10.02(1 \mathrm{H}, \mathrm{s}), 7.42-7.33(5 \mathrm{H}, \mathrm{m}), 7.27(2 \mathrm{H}, \mathrm{m}), 7.10(1 \mathrm{H}, \mathrm{m}), 6.88$ $(2 \mathrm{H}, \mathrm{m}), 5.07(1 \mathrm{H}, \mathrm{dd}, \mathrm{J}=9.9,13.5 \mathrm{~Hz}), 5.00(1 \mathrm{H}, \mathrm{dd}, \mathrm{J}=5.1,13.5 \mathrm{~Hz}), 4.05(1 \mathrm{H}, \mathrm{dd}, \mathrm{J}=$ $5.1,9.9 \mathrm{~Hz}), 1.14(3 \mathrm{H}, \mathrm{s})$. Signals corresponding to the minor diastereomer $\delta 9.59(1 \mathrm{H}$, s), 7.42-7.33 $(5 \mathrm{H}, \mathrm{m}), 7.28(2 \mathrm{H}, \mathrm{m}), 7.09(1 \mathrm{H}, \mathrm{m}), 6.84(2 \mathrm{H}, \mathrm{m}), 5.14(1 \mathrm{H}, \mathrm{dd}, \mathrm{J}=5.1$, $13.2 \mathrm{~Hz}), 5.08(1 \mathrm{H}, \mathrm{dd}, \mathrm{J}=9.9,13.2 \mathrm{~Hz}), 3.91(1 \mathrm{H}, \mathrm{dd}, \mathrm{J}=5.1,9.9 \mathrm{~Hz}), 1.50(3 \mathrm{H}, \mathrm{s}) .{ }^{13} \mathrm{C}$ $\left(100 \mathrm{MHz}, \mathrm{CDCl}_{3}\right)$ : Signals corresponding to the major diastereomer $\delta$ 201.7, 154.0, $134.1,129.5,129.4,128.8,128.6,124.0,121.3,86.5,75.6,49.5,17.0$. Signals corresponding to the minor diastereomer $\delta 203.2,154.2,133.7,129.7,129.5,128.9$, 128.3, 123.5, 119.7, 85.2, 76.0, 51.9, 18.1. IR (neat): 3065 (m), $3036(\mathrm{~m}), 3008(\mathrm{~m}), 2921$ (m), $2817(\mathrm{~m}), 2714(\mathrm{~m}), 1736(\mathrm{~s}), 1590(\mathrm{~s}), 1554(\mathrm{~s}), 1492(\mathrm{~s}), 1455(\mathrm{~m}), 1379(\mathrm{~s}), 1219$ (s), $1109(\mathrm{~m}), 916(\mathrm{~m}), 786(\mathrm{~m}), 759(\mathrm{~m}), 702$ (s). HRMS (ESI): expected for $\left[\mathrm{C}_{17} \mathrm{H}_{17} \mathrm{NO}_{4}+\mathrm{NH}_{4}\right]^{+}: 317.1501$, found: 317.1516 .

\section{Racemic 2-methyl-4-nitro-2-phenoxy-3-phenylbutanal}

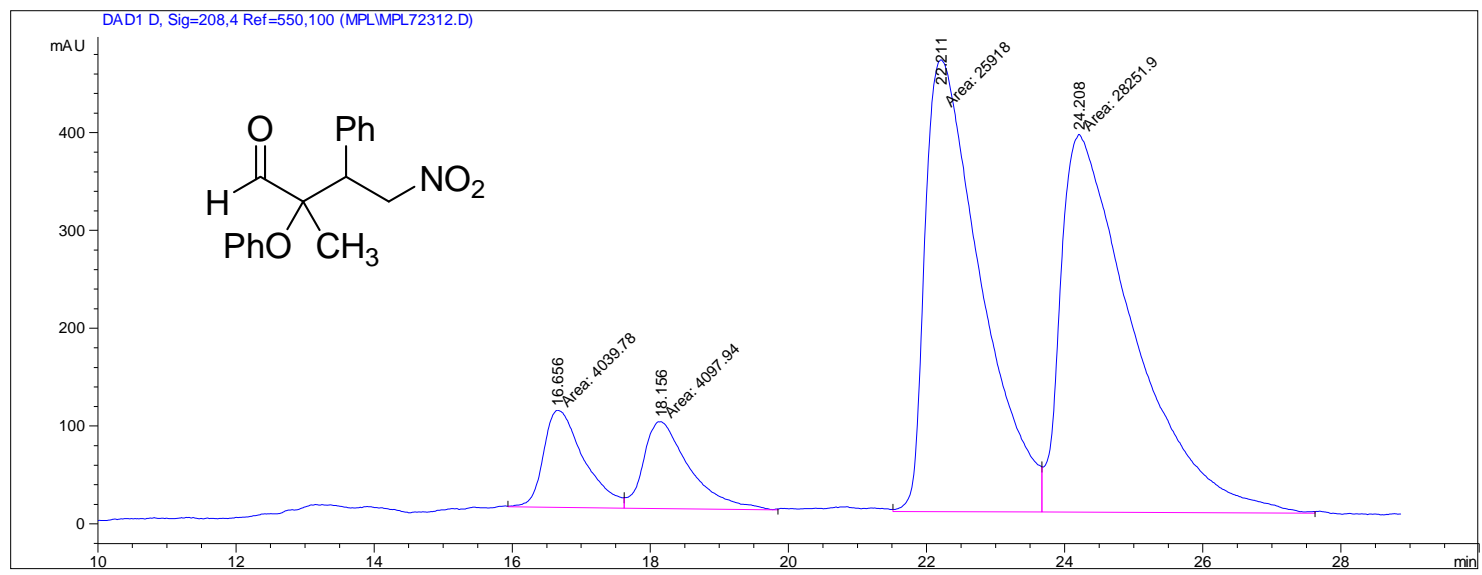

\begin{tabular}{|l|l|l|l|l|l|l|}
\hline $\begin{array}{l}\text { Peak } \\
\#\end{array}$ & $\begin{array}{l}\text { RetTime } \\
{[\mathrm{min}]}\end{array}$ & Type & $\begin{array}{l}\text { Width } \\
{[\mathrm{min}]}\end{array}$ & $\begin{array}{l}\text { Area } \\
{[\mathrm{mAU} * \mathrm{~s}]}\end{array}$ & $\begin{array}{l}\text { Height } \\
{[\mathrm{mAU}]}\end{array}$ & $\begin{array}{l}\text { Area } \\
{[\%]}\end{array}$ \\
\hline 1 & 16.656 & MM & 0.6981 & 4215.40381 & 100.64341 & 6.8115 \\
\hline 2 & 18.156 & MM & 0.7712 & 4133.89746 & 89.33551 & 6.6798 \\
\hline 3 & 22.221 & MM & 0.9340 & $2.58337 \mathrm{e} 4$ & 461.00085 & 41.7439 \\
\hline 4 & 24.208 & MM & 1.1999 & $2.77032 \mathrm{e} 4$ & 384.81418 & 44.7648 \\
\hline Totals: & & & & $6.18862 \mathrm{e} 4$ & 1035.79395 & \\
\hline
\end{tabular}


Enantioenriched (2R,3S)-2-methyl-4-nitro-2-phenoxy-3-phenylbutanal + $(2 S, 3 S)-2-$ methyl-4-nitro-2-phenoxy-3-phenylbutanal

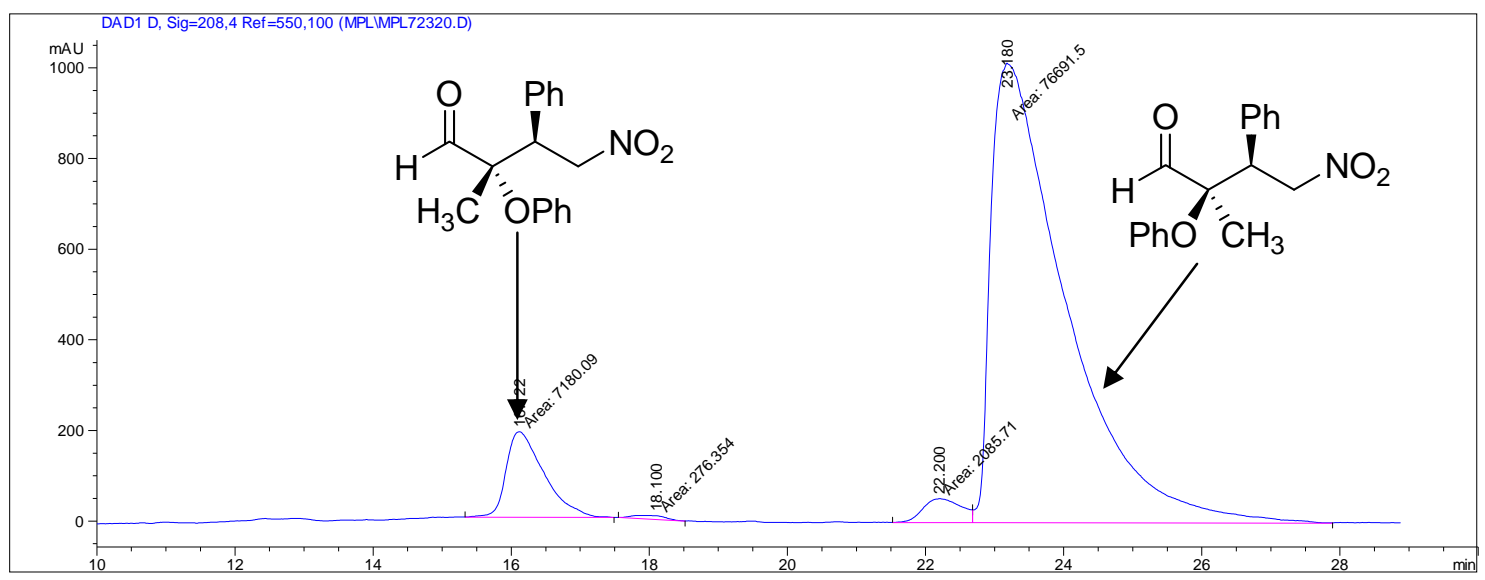

\begin{tabular}{|l|l|l|l|l|l|l|}
\hline $\begin{array}{l}\text { Peak } \\
\#\end{array}$ & $\begin{array}{l}\text { RetTime } \\
{[\mathrm{min}]}\end{array}$ & Type & $\begin{array}{l}\text { Width } \\
{[\mathrm{min}]}\end{array}$ & $\begin{array}{l}\text { Area } \\
{[\mathrm{mAU} * \mathrm{~s}]}\end{array}$ & $\begin{array}{l}\text { Height } \\
{[\mathrm{mAU}]}\end{array}$ & $\begin{array}{l}\text { Area } \\
{[\%]}\end{array}$ \\
\hline 1 & 16.122 & MM & 0.6415 & 7309.00879 & 189.89044 & 8.4384 \\
\hline 2 & 18.100 & MM & 0.5758 & 316.11319 & 9.14928 & 0.3650 \\
\hline 3 & 22.200 & MM & 0.6886 & 2297.62646 & 55.61406 & 2.6526 \\
\hline 4 & 23.180 & MM & 1.2611 & $7.66937 \mathrm{e} 4$ & 1013.59253 & 88.5440 \\
\hline Totals: & & & & $8.66164 \mathrm{e} 4$ & 1268.24632 & \\
\hline
\end{tabular}

(2R,3S)-2-(4-methoxybenzyloxy)-2-methyl-4-nitro-3-phenylbutanal (18): General procedure, chromatography on silica (20\% diethyl ether/hexanes), colorless to light yellow liquid, $78 \%$ yield $(267.9 \mathrm{mg}$ ), $13: 1 \mathrm{dr}, 71 \%$ ee (minor diastereomer), $96 \%$ ee (major diastereomer) as determined by HPLC (Chiralcel OD-H, 5.0\% ethanol/hexanes, $1.0 \mathrm{~mL} / \mathrm{min}, 208 \mathrm{~nm} ; t_{r}($ minor diastereomer $)=21.04 \mathrm{~min}, t_{r}($ minor diastereomer $)=22.63$ $\min , t_{r}($ minor enantiomer, major diastereomer $)=24.16 \mathrm{~min}, t_{r}($ major enantiomer, major diastereomer $)=26.88 \mathrm{~min}) .[\alpha]^{25}=-88.0(\mathrm{c}=0.0202 \mathrm{~g} / 2.0 \mathrm{~mL}$, chloroform $) .{ }^{1} \mathrm{H}$ NMR $\left(400 \mathrm{MHz}, \mathrm{CDCl}_{3}\right): \delta 9.76(1 \mathrm{H}, \mathrm{s}), 7.3(2 \mathrm{H}, \mathrm{m}), 6.93(2 \mathrm{H}, \mathrm{m}), 4.89(1 \mathrm{H}, \mathrm{dd}, \mathrm{J}=10.2$, $13.5 \mathrm{~Hz}), 4.80(1 \mathrm{H}, \mathrm{dd}, \mathrm{J}=4.8,13.5 \mathrm{~Hz}), 4.42(2 \mathrm{H}, \mathrm{s}), 3.87(1 \mathrm{H}, \mathrm{dd}, \mathrm{J}=4.8,10.2$ $\mathrm{Hz}), 3.83(3 \mathrm{H}, \mathrm{s}), 1.24(3 \mathrm{H}, \mathrm{s}) .{ }^{13} \mathrm{C}\left(100 \mathrm{MHz}, \mathrm{CDCl}_{3}\right): \delta 202.3,159.4,134.4,129.5$, 129.4, 129.2, 128.7, 128.4, 113.9, 83.6, 75.8, 66.6, 55.2, 49.1, 16.1. IR (neat): 3064 (m), $3039(\mathrm{~m}), 3003(\mathrm{~m}), 2950(\mathrm{~m}), 2834(\mathrm{~m}), 1731(\mathrm{~s}), 1601(\mathrm{~m}), 1562(\mathrm{~s}), 1512(\mathrm{~s}), 1445$ (m), $1385(\mathrm{~s}), 1307(\mathrm{~m}), 1247(\mathrm{~s}), 1169$ (s), $1130(\mathrm{~s}), 1064(\mathrm{~m}), 1032$ (s), 822 (s), 757 (m), 709 (s). HRMS (ESI): expected for $\left[\mathrm{C}_{19} \mathrm{H}_{21} \mathrm{NO}_{5}+\mathrm{NH}_{4}\right]^{+}: 361.1763$, found: 361.1752 . 
Racemic 2-(4-methoxybenzyloxy)-2-methyl-4-nitro-3-phenylbutanal

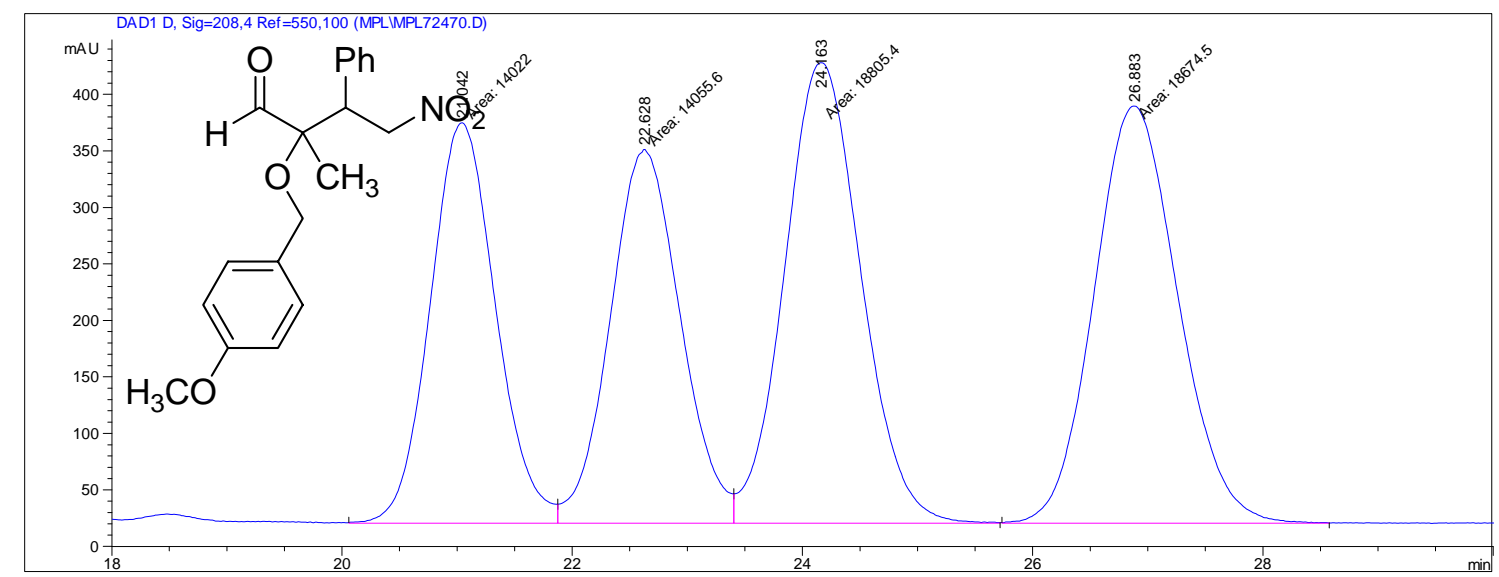

\begin{tabular}{|l|l|l|l|l|l|l|}
\hline $\begin{array}{l}\text { Peak } \\
\#\end{array}$ & $\begin{array}{l}\text { RetTime } \\
{[\mathrm{min}]}\end{array}$ & Type & $\begin{array}{l}\text { Width } \\
{[\mathrm{min}]}\end{array}$ & $\begin{array}{l}\text { Area } \\
{\left[\mathrm{mAU}^{*} \mathrm{~s}\right]}\end{array}$ & $\begin{array}{l}\text { Height } \\
{[\mathrm{mAU}]}\end{array}$ & $\begin{array}{l}\text { Area } \\
{[\%]}\end{array}$ \\
\hline 1 & 21.042 & MF & 0.6592 & $1.40220 \mathrm{e} 4$ & 354.53027 & 21.3889 \\
\hline 2 & 22.628 & MF & 0.7078 & $1.40556 \mathrm{e} 4$ & 330.96271 & 21.4401 \\
\hline 3 & 24.163 & FM & 0.7691 & $1.88054 \mathrm{e} 4$ & 407.52982 & 28.6853 \\
\hline 4 & 26.883 & MM & 0.8430 & $1.86745 \mathrm{e} 4$ & 369.20105 & 28.4857 \\
\hline Totals: & & & & $6.55575 \mathrm{e} 4$ & 1462.22385 & \\
\hline
\end{tabular}

\section{Enantioenriched (2R,3S)-2-(4-methoxybenzyloxy)-2-methyl-4-nitro-3-phenylbutanal}

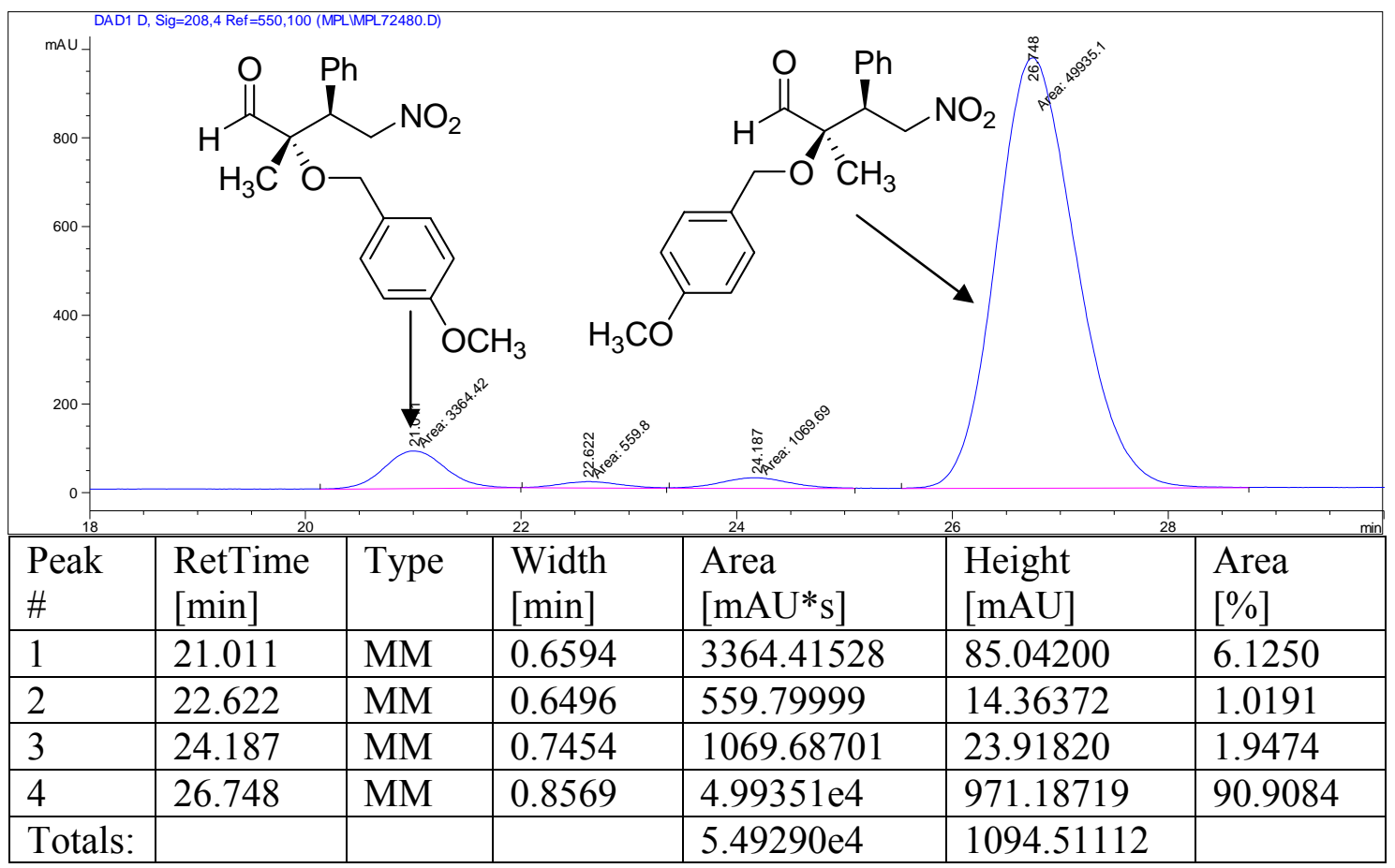

(R)-2-methyl-2-((R)-2-nitro-1-phenylethyl)pentanal + (S)-2-methyl-2-((R)-2-nitro-1- 
phenylethyl)pentanal (19): General procedure, chromatography on silica (10\% diethyl ether/hexanes) diastereomers could not be separated, colorless to light yellow oil, $98 \%$ yield (244.7 mg), 2.1:1 dr, 99\% ee (major diastereomer), $97 \%$ ee (minor diastereomer) as determined by HPLC (Chiralcel OD-H, 5.0\% ethanol/hexane, $1.0 \mathrm{~mL} / \mathrm{min}, 220 \mathrm{~nm}$; $t_{r}$ (major enantiomer, minor diastereomer $)=11.44 \mathrm{~min}, t_{r}$ (major enantiomer, major diastereomer $)=12.38 \mathrm{~min}, t_{r}($ minor enantiomer, major diastereomer $)=15.32 \mathrm{~min}$, $t_{r}($ minor enantiomer, minor diastereomer $\left.)=16.44 \mathrm{~min}\right) .[\alpha]_{\mathrm{D}}^{25}=+19.1(\mathrm{c}=0.0200 \mathrm{~g} / 2.0$ $\mathrm{mL}$, chloroform). ${ }^{1} \mathrm{H}$ NMR (400 $\mathrm{MHz}, \mathrm{CDCl}_{3}$ ): Signals corresponding to the major diastereomer $\delta 9.52(1 \mathrm{H}, \mathrm{s}), 7.30(5 \mathrm{H}, \mathrm{m}), 4.83(1 \mathrm{H}, \mathrm{dd}, \mathrm{J}=11.3,13.2 \mathrm{~Hz}), 4.62(1 \mathrm{H}, \mathrm{dd}$, $\mathrm{J}=4.0,13.2 \mathrm{~Hz}), 3.79(1 \mathrm{H}, \mathrm{dd}, \mathrm{J}=4.0,11.3 \mathrm{~Hz}), 1.46(1 \mathrm{H}, \mathrm{m}), \delta 1.32(1 \mathrm{H}, \mathrm{m}), 1.20(2 \mathrm{H}$, $\mathrm{m}), 1.10(3 \mathrm{H}, \mathrm{s}), 0.82(3 \mathrm{H}, \mathrm{t}, \mathrm{J}=7.3 \mathrm{~Hz})$. Signals to the major diastereomer $\delta 9.50(1 \mathrm{H}$, s), $7.18(5 \mathrm{H}, \mathrm{m}), 4.85(1 \mathrm{H}, \mathrm{dd}, \mathrm{J}=11.3,13.2 \mathrm{~Hz}), 4.75(1 \mathrm{H}, \mathrm{dd}, \mathrm{J}=4.4,13.2 \mathrm{~Hz}), 3.77$ $(1 \mathrm{H}, \mathrm{dd}, \mathrm{J}=4.411 .3 \mathrm{~Hz}), 1.62(1 \mathrm{H}, \mathrm{m}), 1.46(1 \mathrm{H}, \mathrm{m}), 1.20(2 \mathrm{H}, \mathrm{m}), 1.08(3 \mathrm{H}, \mathrm{s}), 0.89$ $(3 \mathrm{H}, \mathrm{t}, \mathrm{J}=7.3 \mathrm{~Hz}) \cdot{ }^{13} \mathrm{C}\left(100 \mathrm{MHz}, \mathrm{CDCl}_{3}\right)$ : Signals corresponding to the major diastereomer $\delta 205.3,135.2,129.1,128.6,128.0,76.6,51.6,47.5,37.5,17.0,15.7,14.4$. Signals corresponding to the minor diastereomer $\delta 204.9,135.2,129.0,128.6,128.1,76.2$, 51.0, 49.1, 36.4, 17.2, 17.0, 14.5. IR (neat): 3032 (m), 2962 (s), 2935 (s), 2875 (s), 2720 (m), $1723(\mathrm{~s}), 1556(\mathrm{~s}), 1497(\mathrm{~m}), 1455(\mathrm{~s}), 1436(\mathrm{~m}), 1379(\mathrm{~s}), 1203(\mathrm{~m}), 1090(\mathrm{~m}), 913$ (m), $751(\mathrm{~m}), 705$ (s). HRMS (ESI): expected for $\left[\mathrm{C}_{14} \mathrm{H}_{19} \mathrm{NO}_{3}+\mathrm{NH}_{4}\right]^{+}: 267.1709$, found: 267.1711.

\section{Racemic 2-methyl-2-(2-nitro-1-phenylethyl)pentanal}

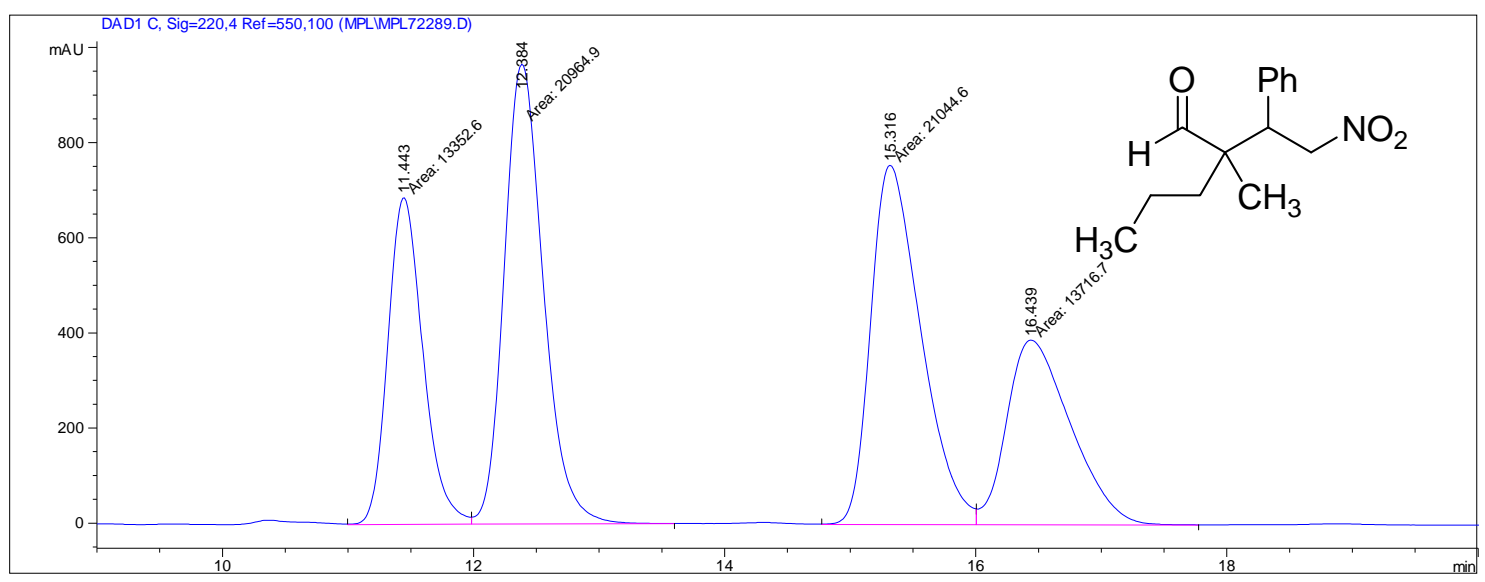

\begin{tabular}{|l|l|l|l|l|l|l|}
\hline $\begin{array}{l}\text { Peak } \\
\#\end{array}$ & $\begin{array}{l}\text { RetTime } \\
{[\mathrm{min}]}\end{array}$ & Type & $\begin{array}{l}\text { Width } \\
{[\mathrm{min}]}\end{array}$ & $\begin{array}{l}\text { Area } \\
{\left[\mathrm{mAU}{ }^{*}\right]}\end{array}$ & $\begin{array}{l}\text { Height } \\
{[\mathrm{mAU}]}\end{array}$ & $\begin{array}{l}\text { Area } \\
{[\%]}\end{array}$ \\
\hline 1 & 11.443 & MF & 0.3237 & $1.33526 \mathrm{e} 4$ & 687.59418 & 19.3295 \\
\hline 2 & 12.384 & FM & 0.3612 & $2.09649 \mathrm{e} 4$ & 967.24445 & 30.3492 \\
\hline 3 & 15.316 & MF & 0.4643 & $2.10446 \mathrm{e} 4$ & 755.43335 & 30.4646 \\
\hline 4 & 16.439 & FM & 0.5888 & $1.37167 \mathrm{e} 4$ & 388.26694 & 19.8566 \\
\hline Totals: & & & & $6.90788 \mathrm{e} 4$ & 2798.53891 & \\
\hline
\end{tabular}


Enantioenriched $(R)$-2-methyl-2-((R)-2-nitro-1-phenylethyl)pentanal + (S)-2-methyl2-((R)-2-nitro-1-phenylethyl)pentanal

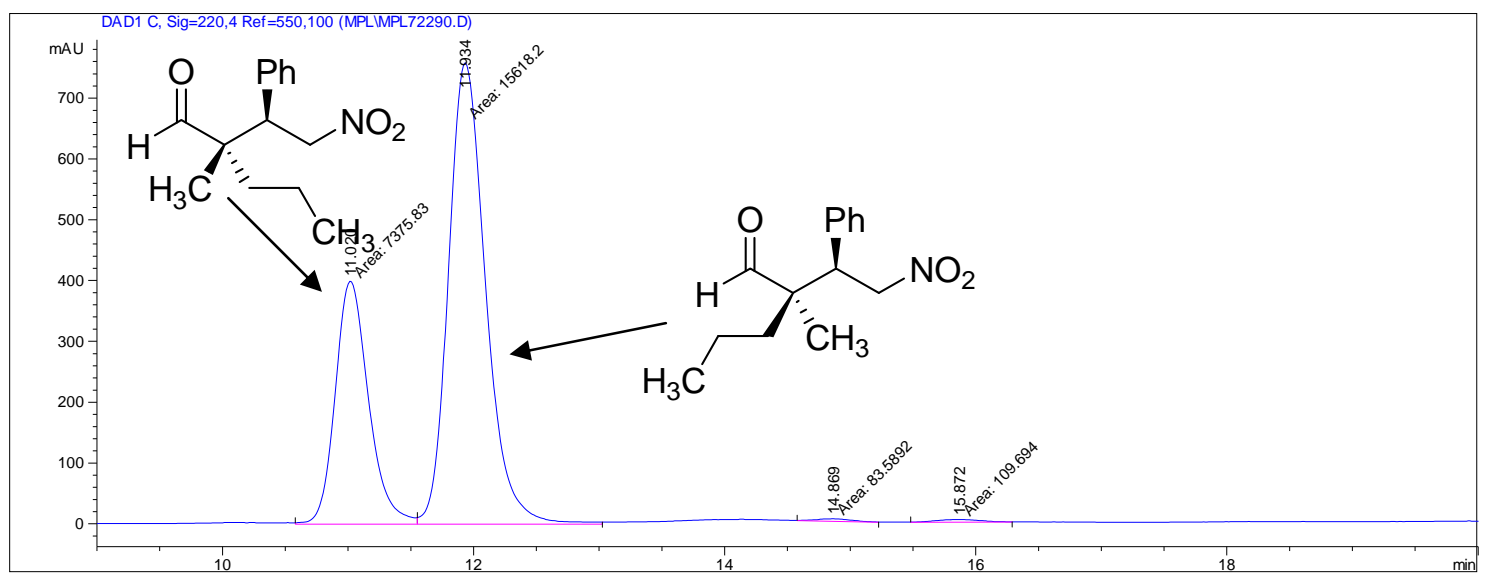

\begin{tabular}{|l|l|l|l|l|l|l|}
\hline $\begin{array}{l}\text { Peak } \\
\#\end{array}$ & $\begin{array}{l}\text { RetTime } \\
{[\mathrm{min}]}\end{array}$ & Type & $\begin{array}{l}\text { Width } \\
{[\mathrm{min}]}\end{array}$ & $\begin{array}{l}\text { Area } \\
{\left[\mathrm{mAU}^{*} \mathrm{~s}\right]}\end{array}$ & $\begin{array}{l}\text { Height } \\
{[\mathrm{mAU}]}\end{array}$ & $\begin{array}{l}\text { Area } \\
{[\%]}\end{array}$ \\
\hline 1 & 11.020 & MF & 0.3073 & 7375.82764 & 687.59418 & 31.8098 \\
\hline 2 & 11.934 & FM & 0.3429 & $1.56182 \mathrm{e} 4$ & 967.24445 & 67.3567 \\
\hline 3 & 14.869 & MM & 0.3403 & 83.58918 & 755.43335 & 0.3605 \\
\hline 4 & 15.872 & MM & 0.4016 & 109.69353 & 388.26694 & 0.4731 \\
\hline Totals: & & & & $2.31873 \mathrm{e} 4$ & 2798.53891 & \\
\hline
\end{tabular}

(2R,3R)-2-(benzo[d][1,3]dioxol-5-ylmethyl)-2-methyl-4-nitro-3-phenylbutanal + (2S,3R)-2-(benzo[d][1,3]dioxol-5-ylmethyl)-2-methyl-4-nitro-3-phenylbutanal (20): General procedure, chromatography on silica ( $20 \%$ diethyl ether/hexanes) diastereomers could not be separated, white solid, m.p. $=136{ }^{\circ} \mathrm{C}, 82 \%$ yield $(278.8 \mathrm{mg}), 3.1: 1 \mathrm{dr}, 99 \%$ ee (major diastereomer), $99 \%$ ee (minor diastereomer) as determined by SFC (Chiralcel $\mathrm{OD}-\mathrm{H}, 3.0 \%$ methanol $/ \mathrm{CO}_{2}, 3.0 \mathrm{~mL} / \mathrm{min}, 208 \mathrm{~nm}, 30{ }^{\circ} \mathrm{C} ; t_{r}$ (major enantiomer, major diastereomer $)=28.47 \mathrm{~min}, t_{r}($ minor enantiomer, major diastereomer $)=34.67 \mathrm{~min}$, $t_{r}($ major enantiomer, minor diastereomer $)=42.29 \mathrm{~min}, t_{r}($ minor enantiomer, minor diastereomer $)=45.25 \mathrm{~min}) .[\alpha]_{\mathrm{D}}^{25}=+25.3\left(\mathrm{c}=0.0212 \mathrm{~g} / 2.0 \mathrm{~mL}\right.$, chloroform). ${ }^{1} \mathrm{H}$ NMR $\left(400 \mathrm{MHz}, \mathrm{CDCl}_{3}\right)$ : Signals corresponding to the major diastereomer $\delta 9.62(1 \mathrm{H}, \mathrm{s}), 7.34$ $(3 \mathrm{H}, \mathrm{m}), 7.22(2 \mathrm{H}, \mathrm{m}), 6.68(1 \mathrm{H}, \mathrm{d}, \mathrm{J}=7.7 \mathrm{~Hz}), 6.43(2 \mathrm{H}, \mathrm{m}), 5.91(2 \mathrm{H}, \mathrm{s}), 4.90(1 \mathrm{H}, \mathrm{dd}$, $\mathrm{J}=11.7,13.2 \mathrm{~Hz}), 4.64(1 \mathrm{H}, \mathrm{dd}, \mathrm{J}=4.0,13.2 \mathrm{~Hz}), 3.82(1 \mathrm{H}, \mathrm{dd}, \mathrm{J}=4.0,11.7 \mathrm{~Hz}), 3.01$ $(1 \mathrm{H}, \mathrm{d}, \mathrm{J}=13.7 \mathrm{~Hz}), 2.32(1 \mathrm{H}, \mathrm{d}, \mathrm{J}=13.7 \mathrm{~Hz}), 1.06(3 \mathrm{H}, \mathrm{s})$. Signals corresponding to the minor diastereomer $\delta 9.59(1 \mathrm{H}, \mathrm{s}), 7.34(3 \mathrm{H}, \mathrm{m}), 7.22(2 \mathrm{H}, \mathrm{m}), 6.69(1 \mathrm{H}, \mathrm{d}, \mathrm{J}=7.7 \mathrm{~Hz})$, $6.49(2 \mathrm{H}, \mathrm{m}), 5.91(2 \mathrm{H}, \mathrm{s}), 4.82(2 \mathrm{H}, \mathrm{m}), 3.83(1 \mathrm{H}, \mathrm{m}), 3.04(1 \mathrm{H}, \mathrm{d}, \mathrm{J}=13.5 \mathrm{~Hz}), 2.67$ $(1 \mathrm{H}, \mathrm{d}, \mathrm{J}=13.5 \mathrm{~Hz}), 1.25(3 \mathrm{H}, \mathrm{s}) .{ }^{13} \mathrm{C}\left(100 \mathrm{MHz}, \mathrm{CDCl}_{3}\right)$ : Signals corresponding to the major diastereomer $\delta 205.8,147.6,135.1,129.3,128.8,128.6,128.3,123.3,110.4,108.2$, $101.0,76.7,52.3,48.7,42.1,17.8$. Signals corresponding to the minor diastereomer $\delta$ 204.6, 146.6, 135.0, 129.2, 129.2, 129.0, 128.9, 128.4, 123.4, 110.5, 108.2, 101.0, 76.2, 51.8, 49.5, 40.3, 17.8. IR (neat): 3031 (w), 2982 (w), 2919 (w), 2778 (w), 2724 (w), 1723 
(m), 1555 (s), 1490 (s), 1455 (m), 1379 (m), 1252 (s), 1039 (s), 929 (m), 752 (m), 705 (m). HRMS (ESI): expected for $\left[\mathrm{C}_{19} \mathrm{H}_{19} \mathrm{NO}_{5}+\mathrm{NH}_{4}\right]^{+}: 359.1607$, found: 359.1592 .

\section{Racemic 2-(benzo[d][1,3]dioxol-5-ylmethyl)-2-methyl-4-nitro-3-phenylbutanal}

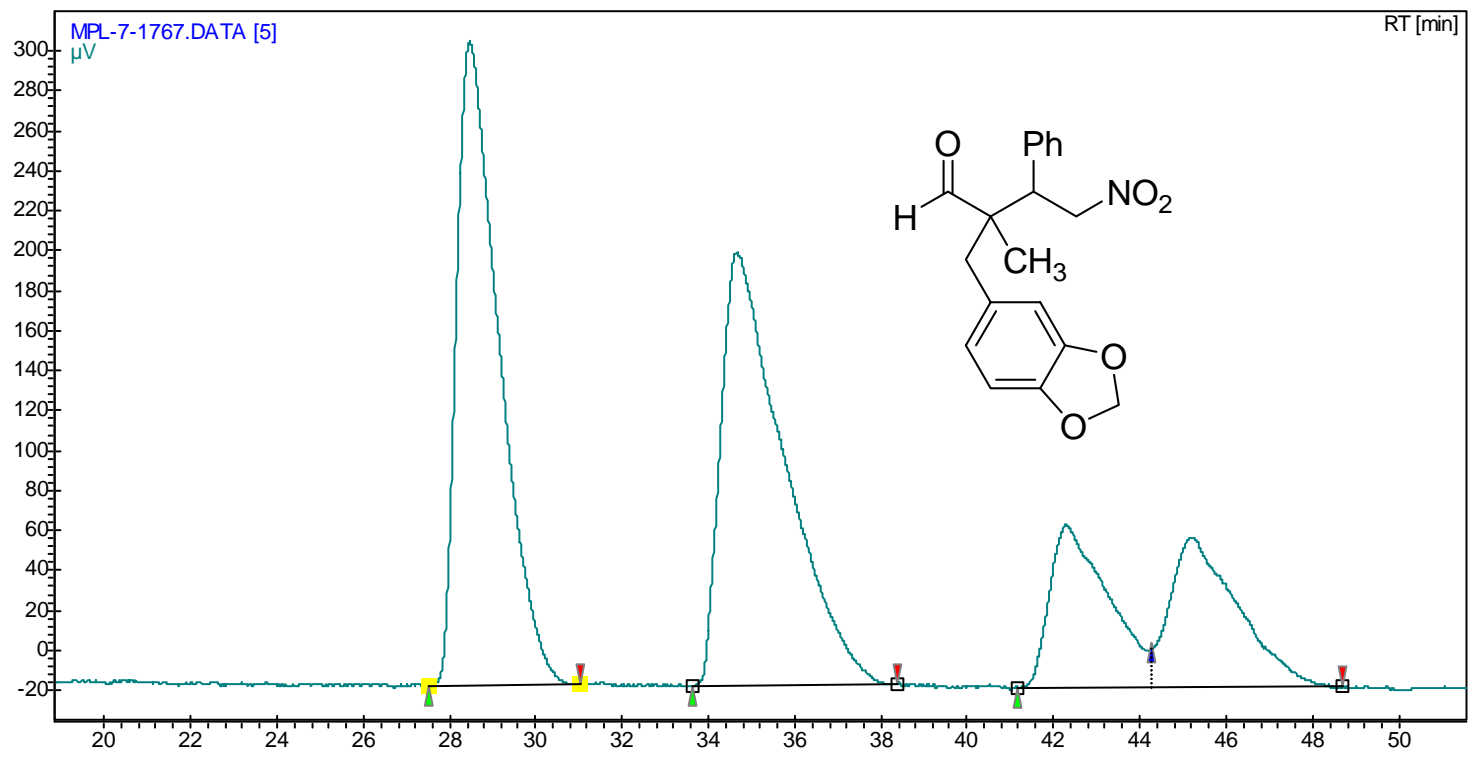

\begin{tabular}{|l|l|l|l|l|}
\hline$\#$ & Time $[\mathrm{Min}]$ & Height $[\mu \mathrm{V}]$ & Area $[\mu \mathrm{V} . \mathrm{Min}]$ & Area $[\%]$ \\
\hline 1 & 28.47 & 321.9 & 367.9 & 36.752 \\
\hline 2 & 34.67 & 216.5 & 368.1 & 36.774 \\
\hline 3 & 42.29 & 81.4 & 128.7 & 12.858 \\
\hline 4 & 45.25 & 75.0 & 136.3 & 13.616 \\
\hline & & & & \\
\hline Total & & 694.8 & 1001.0 & 100.000 \\
\hline
\end{tabular}




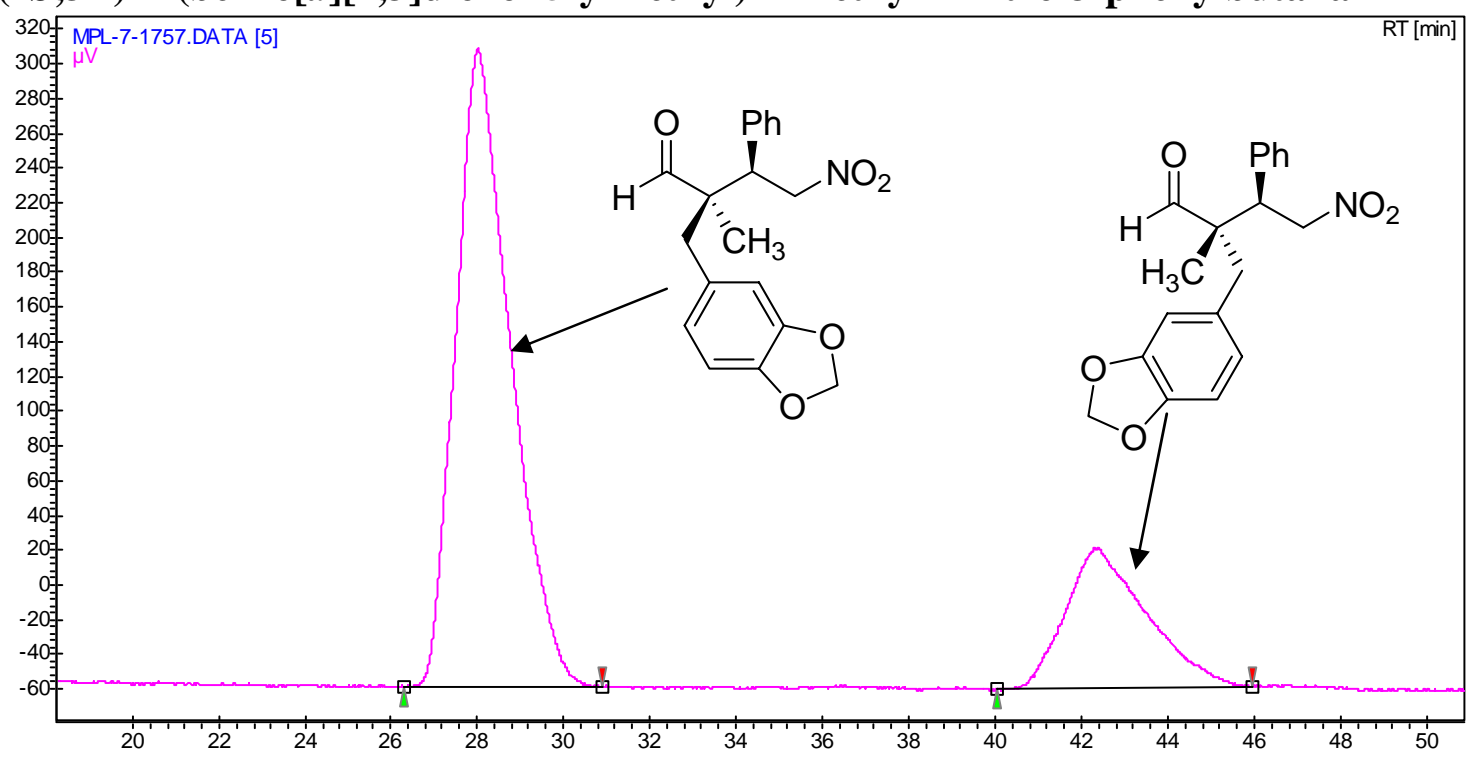

\begin{tabular}{|l|l|l|l|l|}
\hline$\#$ & Time $[\mathrm{Min}]$ & Height $[\mu \mathrm{V}]$ & Area $[\mu \mathrm{V} . \mathrm{Min}]$ & Area $[\%]$ \\
\hline 1 & 28.01 & 367.0 & 539.0 & 75.748 \\
\hline 2 & 42.39 & 80.3 & 172.6 & 24.252 \\
\hline & & & & \\
\hline Total & & 447.2 & 711.5 & 100.000 \\
\hline
\end{tabular}

(2R,3R)-2-(benzo[d][1,3]dioxol-5-ylmethyl)-2-methyl-4-nitro-3-(2-

(trifluoromethyl)phenyl)butanal $+(2 R, 3 R)-2-($ benzo[d][1,3]dioxol-5-ylmethyl)-2methyl-4-nitro-3-(2-(trifluoromethyl)phenyl)butanal (21): General procedure, chromatography on silica (20\% diethyl ether/hexanes) diastereomers could not be separated, white solid, m.p. $=128-130{ }^{\circ} \mathrm{C}, 63 \%$ yield $(258.6 \mathrm{mg}), 4.7: 1 \mathrm{dr}, 98 \%$ ee (major diastereomer), $93 \%$ ee (minor diastereomer) as determined by HPLC (Chiralcel OD-H, $10.0 \%$ ethanol $/$ hexane, $1.0 \mathrm{~mL} / \mathrm{min}, 208 \mathrm{~nm} ; t_{r}$ (major enantiomer, major diastereomer $)=$ $23.28 \mathrm{~min}, t_{r}($ minor enantiomer, minor diastereomer $)=25.83 \mathrm{~min}, t_{r}$ (major enantiomer, minor diastereomer $)=28.50 \mathrm{~min}, t_{r}($ minor enantiomer, major diastereomer $)=31.86$ $\min ) .[\alpha]_{D}^{25}=-10.8(\mathrm{c}=0.0202 \mathrm{~g} / 2.0 \mathrm{~mL}$, chloroform $) .{ }^{1} \mathrm{H}$ NMR $\left(400 \mathrm{MHz}, \mathrm{CDCl}_{3}\right)$ : Signals corresponding to the major diastereomer $\delta 9.62(1 \mathrm{H}, \mathrm{s}), 7.72-7.36(4 \mathrm{H}, \mathrm{m}), 6.72$ $(1 \mathrm{H}, \mathrm{m}), 6.54(2 \mathrm{H}, \mathrm{m}), 5.92(2 \mathrm{H}, \mathrm{s}), 4.93(1 \mathrm{H}, \mathrm{dd}, \mathrm{J}=10.2,11.7 \mathrm{~Hz}), 4.83(1 \mathrm{H}, \mathrm{dd}, \mathrm{J}=$ 3.7, $11.7 \mathrm{~Hz}), 4.24(1 \mathrm{H}, \mathrm{dd}, \mathrm{J}=3.7,10.2 \mathrm{~Hz}), 3.07(1 \mathrm{H}, \mathrm{d}, \mathrm{J}=13.7 \mathrm{~Hz}), 2.49(1 \mathrm{H}, \mathrm{d}, \mathrm{J}=$ $13.9 \mathrm{~Hz}), 1.00(3 \mathrm{H}, \mathrm{s})$. Signals corresponding to the minor diastereomer $\delta 9.52(1 \mathrm{H}, \mathrm{s})$, 7.72-7.36 $(4 \mathrm{H}, \mathrm{m}), 6.71(1 \mathrm{H}, \mathrm{m}), 6.54(2 \mathrm{H}, \mathrm{m}), 4.80(1 \mathrm{H}, \mathrm{dd}, \mathrm{J}=4.4,12.1), 4.75(1 \mathrm{H}, \mathrm{dd}$, $\mathrm{J}=9.9,12.1 \mathrm{~Hz}), 4.28(1 \mathrm{H}, \mathrm{dd}, \mathrm{J}=4.4,9.9 \mathrm{~Hz}), 2.91(1 \mathrm{H}, \mathrm{d}, \mathrm{J}=13.9 \mathrm{~Hz}), 2.77(1 \mathrm{H}, \mathrm{d}, \mathrm{J}$ $=13.9 \mathrm{~Hz}), 1.18(3 \mathrm{H}, \mathrm{s}) \cdot{ }^{13} \mathrm{C}\left(100 \mathrm{MHz}, \mathrm{CDCl}_{3}\right)$ : Signals corresponding to the major diastereomer $\delta$ 205.3, 147.6, 146.7, 135.9, 132.4, $130.2(1 \mathrm{C}, \mathrm{q}, \mathrm{J}=29.0 \mathrm{~Hz}), 129.2$, 128.2, 128.1, $127.3(1 \mathrm{C}, \mathrm{q}, \mathrm{J}=6.1 \mathrm{~Hz}), 124.0(1 \mathrm{C}, \mathrm{q}, \mathrm{J}=274.7 \mathrm{~Hz}), 123.6,110.5,108.2$, 
101.0, 78.2, 53.0, 43.6, 41.5, 17.2. Signals corresponding to the minor diastereomer $\delta$ 203.8, 147.7, 146.7, 153.2, 132.5, 130.2 (1C, q, J = 29.0 Hz), 128.9, 128.5, 128.4, 127.0 $(1 \mathrm{C}, \mathrm{q}, \mathrm{J}=6.1 \mathrm{~Hz}), 124.0(1 \mathrm{C}, \mathrm{q}, \mathrm{J}=274.7 \mathrm{~Hz}), 123.5,110.5,108.2$, 101.0, 77.6, 52.2, 44.0, 41.3, 17.8. IR (neat): $3078(w), 2991(w), 2904(w), 2779(w), 2730(w), 1725(\mathrm{~m})$, 1491 (s), 1454 (m), 1379 (m), 1310 (s), 1253 (s), 1163 (m), 1122 (s), 1037 (s), 929 (m), $772(\mathrm{~m}), 733$ (m). HRMS (ESI): expected for $\left[\mathrm{C}_{20} \mathrm{H}_{18} \mathrm{~F}_{3} \mathrm{NO}_{5}+\mathrm{NH}_{4}\right]^{+}$: 427.1481, found: 427.1501 .

Racemic2-(benzo[d][1,3]dioxol-5-ylmethyl)-2-methyl-4-nitro-3-(2-(trifluoromethyl) phenyl)butanal

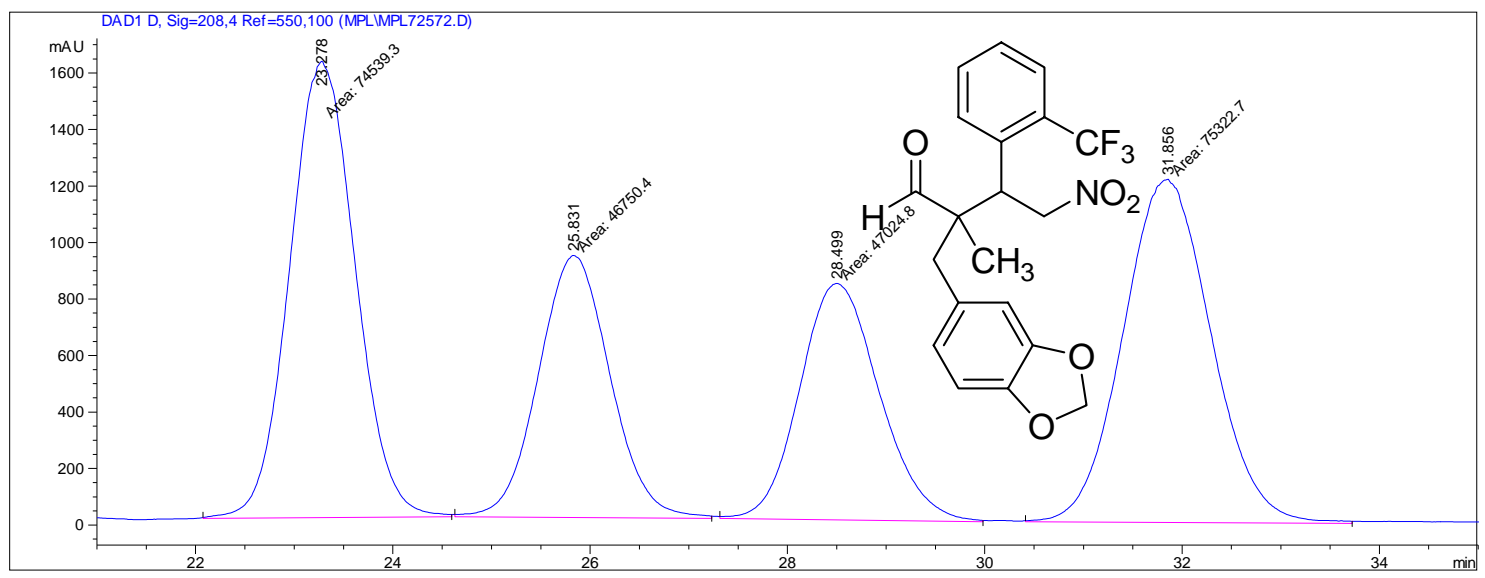

\begin{tabular}{|l|l|l|l|l|l|l|}
\hline $\begin{array}{l}\text { Peak } \\
\#\end{array}$ & $\begin{array}{l}\text { RetTime } \\
{[\mathrm{min}]}\end{array}$ & Type & $\begin{array}{l}\text { Width } \\
{[\mathrm{min}]}\end{array}$ & $\begin{array}{l}\text { Area } \\
{[\mathrm{mAU} \text { s }]}\end{array}$ & $\begin{array}{l}\text { Height } \\
{[\mathrm{mAU}]}\end{array}$ & $\begin{array}{l}\text { Area } \\
{[\%]}\end{array}$ \\
\hline 1 & 23.278 & MM & 0.7687 & $7.45393 \mathrm{e} 4$ & 1616.07373 & 30.5944 \\
\hline 2 & 25.831 & MM & 0.8398 & $4.67504 \mathrm{e} 4$ & 927.86145 & 19.1885 \\
\hline 3 & 28.499 & MM & 0.9367 & $4.70248 \mathrm{e} 4$ & 836.73743 & 19.3012 \\
\hline 4 & 31.856 & MM & 1.0328 & $7.53227 \mathrm{e} 4$ & 1215.49036 & 30.9159 \\
\hline Totals: & & & & $2.43637 \mathrm{e} 5$ & 4596.16296 & \\
\hline
\end{tabular}


(2R,3R)-2-(benzo[d][1,3]dioxol-5-ylmethyl)-2-methyl-4-nitro-3-(2-(trifluoromethyl) phenyl)butanal + $(2 R, 3 R)$-2-(benzo[d][1,3]dioxol-5-ylmethyl)-2-methyl-4-nitro-3-(2(trifluoromethyl)phenyl)butanal

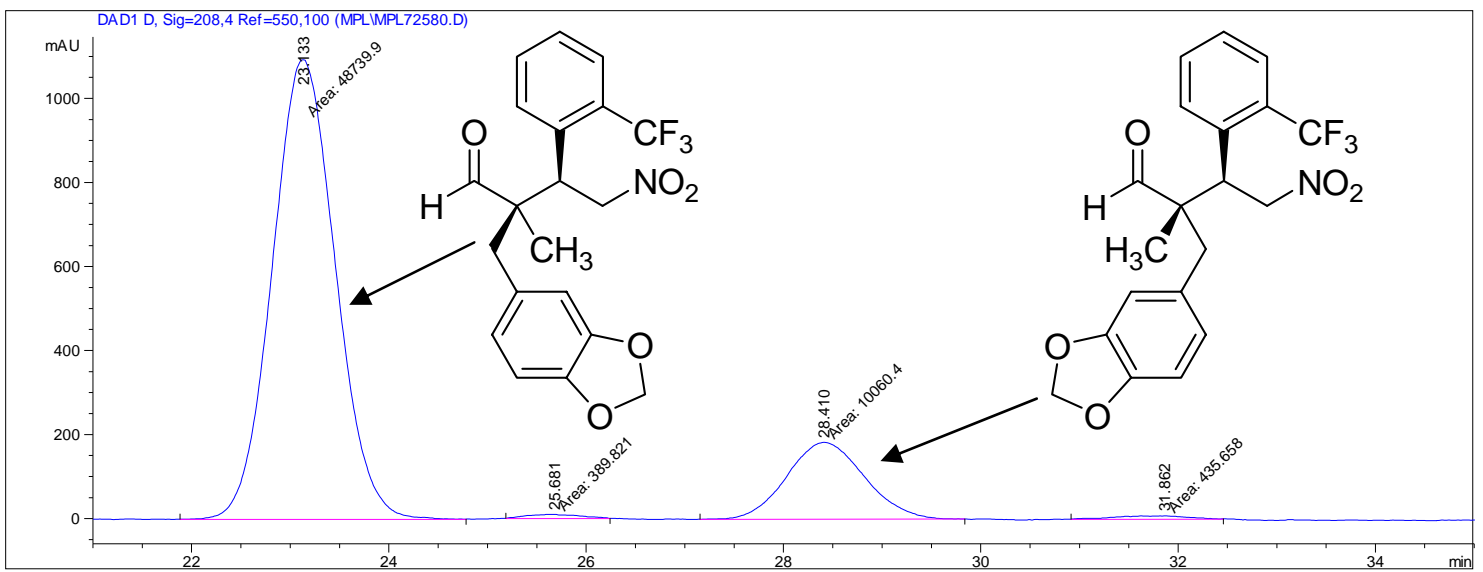

\begin{tabular}{|l|l|l|l|l|l|l|}
\hline $\begin{array}{l}\text { Peak } \\
\#\end{array}$ & $\begin{array}{l}\text { RetTime } \\
{[\mathrm{min}]}\end{array}$ & Type & $\begin{array}{l}\text { Width } \\
{[\mathrm{min}]}\end{array}$ & $\begin{array}{l}\text { Area } \\
{\left[\mathrm{mAU}^{*} \mathrm{~s}\right]}\end{array}$ & $\begin{array}{l}\text { Height } \\
{[\mathrm{mAU}]}\end{array}$ & $\begin{array}{l}\text { Area } \\
{[\%]}\end{array}$ \\
\hline 1 & 23.133 & MM & 0.7421 & $4.87399 \mathrm{e} 4$ & 1094.68542 & 81.7431 \\
\hline 2 & 25.681 & MM & 0.6496 & 389.82111 & 10.00170 & 0.6538 \\
\hline 3 & 28.410 & MM & 0.9139 & $1.00604 \mathrm{e} 4$ & 183.47906 & 16.8725 \\
\hline 4 & 31.862 & MM & 0.8988 & 435.65826 & 8.07817 & 0.7307 \\
\hline Totals: & & & & $5.96258 \mathrm{e} 4$ & 1296.24436 & \\
\hline
\end{tabular}

(2R,3R)-2-(benzo[d][1,3]dioxol-5-ylmethyl)-3-(4-bromophenyl)-2-methyl-4nitrobutanal + (2R,3R)-2-(benzo[d][1,3]dioxol-5-ylmethyl)-3-(4-bromophenyl)-2methyl-4-nitrobutanal (22): General procedure, chromatography on silica (20\% diethyl ether/hexanes) diastereomers could not be separated, white solid, m.p. $=166-168{ }^{\circ} \mathrm{C}$, $81 \%$ yield (340.3 mg), 3.8:1 dr, 99\% ee (major diastereomer), $99 \%$ ee (minor diastereomer) as determined by HPLC (Chiralcel OD-H, 25.0\% isopropanol/hexanes, 1.0 $\mathrm{mL} / \mathrm{min}, 208 \mathrm{~nm}$; $t_{r}$ (major enantiomer, major diastereomer) $=30.64 \mathrm{~min}, t_{r}($ minor enantiomer, major diastereomer $)=34.82 \mathrm{~min}, t_{r}$ (major enantiomer, minor diastereomer) $=39.79 \mathrm{~min}, t_{r}($ minor enantiomer, minor diastereomer $\left.)=43.33 \mathrm{~min}\right) .[\alpha]_{\mathrm{D}}^{25}=+15.9(\mathrm{c}=$ $0.0201 \mathrm{~g} / 2.0 \mathrm{~mL}$, chloroform). ${ }^{1} \mathrm{H}$ NMR (400 MHz, $\mathrm{CDCl}_{3}$ ): Signals corresponding to the major diastereomer $\delta 9.57(1 \mathrm{H}, \mathrm{s}), 7.47(2 \mathrm{H}, \mathrm{d}, \mathrm{J}=8.4 \mathrm{~Hz}), 7.08(2 \mathrm{H}, \mathrm{d}, \mathrm{J}=8.4 \mathrm{~Hz}), 6.67$ $(1 \mathrm{H}, \mathrm{d}, \mathrm{J}=7.7 \mathrm{~Hz}), 6.44-6.41(2 \mathrm{H}, \mathrm{m}), 5.91(2 \mathrm{H}, \mathrm{s}), 4.85(1 \mathrm{H}, \mathrm{dd}, \mathrm{J}=11.7,13.2 \mathrm{~Hz})$, $4.63(1 \mathrm{H}, \mathrm{dd}, \mathrm{J}=4.0,13.2 \mathrm{~Hz}), 3.76(1 \mathrm{H}, \mathrm{dd}, \mathrm{J}=4.0,11.7 \mathrm{~Hz}), 2.95(1 \mathrm{H}, \mathrm{d}, \mathrm{J}=13.9 \mathrm{~Hz})$, $2.33(1 \mathrm{H}, \mathrm{d}, \mathrm{J}=13.9 \mathrm{~Hz}), 1.03(3 \mathrm{H}, \mathrm{s})$. Signals corresponding to the minor diastereomer $\delta$ $9.52(1 \mathrm{H}, \mathrm{s}), 7.47(2 \mathrm{H}, \mathrm{d}, \mathrm{J}=8.4 \mathrm{~Hz}), 7.08(2 \mathrm{H}, \mathrm{d}, \mathrm{J}=8.4 \mathrm{~Hz}), 6.68(1 \mathrm{H}, \mathrm{d}, \mathrm{J}=7.7 \mathrm{~Hz})$, 6.49-6.44 (2H, m), $5.91(2 \mathrm{H}, \mathrm{s}), 4.78(2 \mathrm{H}, \mathrm{m}), 3.78(1 \mathrm{H}, \mathrm{m}), 2.99(1 \mathrm{H}, \mathrm{d}, \mathrm{J}=13.5 \mathrm{~Hz})$, $2.62(1 \mathrm{H}, \mathrm{d}, 13.5 \mathrm{~Hz}), 1.05(3 \mathrm{H}, \mathrm{s}) \cdot{ }^{13} \mathrm{C}\left(100 \mathrm{MHz}, \mathrm{CDCl}_{3}\right)$ : Signals corresponding to the 
major enantiomer $\delta 205.5,147.7,146.7,134.3,132.0,130.9,123.4,122.5,110.4,108.2$, $101.1,76.4,52.1,48.2,42.1,16.2$. Signals corresponding to the minor diastereomer $\delta$ 204.3, 147.6, 146.7, 134.1, 132.1, 130.8, 128.6, 128.2, 127.6, 110.4, 108.2, 101.1, 75.9, 51.7, 48.8, 40.3, 17.9. IR (neat): 3026 (w), 2980 (w), 2917 (w), 2778 (w), 2725 (w), 1723 (m), 1555 (s), 1490 (s), 1443 (m), 1378 (m), 1251 (m), 1039 (m), 1011 (m), 929 (m), 814 (m), 757 (m). HRMS (ESI): expected for $\left[\mathrm{C}_{19} \mathrm{H}_{18} \mathrm{BrNO}_{5}+\mathrm{NH}_{4}\right]^{+}$: 437.0712, 439.0692, found: $437.0721,439.0699$.

\section{Racemic 2-(benzo[d][1,3]dioxol-5-ylmethyl)-3-(4-bromophenyl)-2-methyl-4- nitrobutanal}

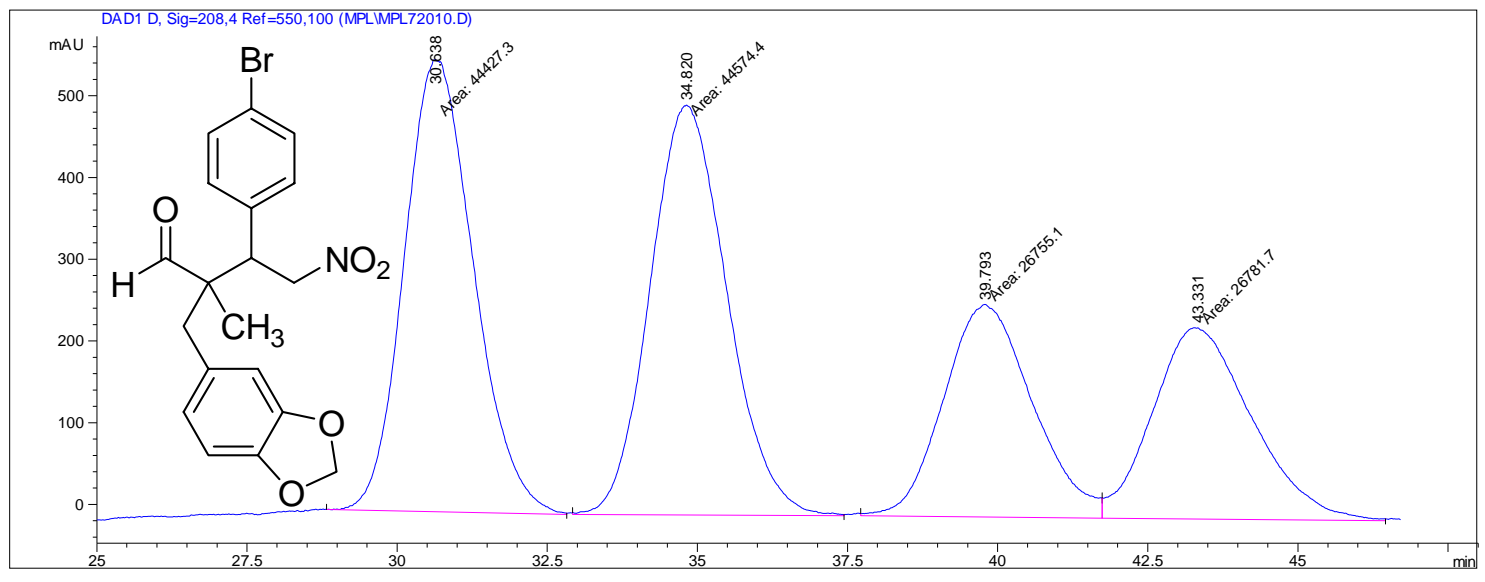

\begin{tabular}{|l|l|l|l|l|l|l|}
\hline $\begin{array}{l}\text { Peak } \\
\#\end{array}$ & $\begin{array}{l}\text { RetTime } \\
{[\mathrm{min}]}\end{array}$ & Type & $\begin{array}{l}\text { Width } \\
{[\mathrm{min}]}\end{array}$ & $\begin{array}{l}\text { Area } \\
{[\mathrm{mAU} * \mathrm{~s}]}\end{array}$ & $\begin{array}{l}\text { Height } \\
{[\mathrm{mAU}]}\end{array}$ & $\begin{array}{l}\text { Area } \\
{[\%]}\end{array}$ \\
\hline 1 & 30.638 & MM & 1.3348 & $4.43107 \mathrm{e} 4$ & 553.27155 & 12.2017 \\
\hline 2 & 34.820 & MM & 1.4722 & $4.42024 \mathrm{e} 4$ & 500.41241 & 38.7498 \\
\hline 3 & 39.793 & MF & 1.7061 & $2.64750 \mathrm{e} 4$ & 258.63345 & 37.0904 \\
\hline 4 & 43.331 & FM & 1.8820 & $2.62786 \mathrm{e} 4$ & 232.71701 & 11.9581 \\
\hline Totals: & & & & $1.41267 \mathrm{e} 5$ & 1545.03442 & \\
\hline
\end{tabular}


Enantioenriched (2R,3R)-2-(benzo[d][1,3]dioxol-5-ylmethyl)-3-(4-bromophenyl)-2methyl-4-nitrobutanal + (2R,3R)-2-(benzo[d][1,3]dioxol-5-ylmethyl)-3-(4bromophenyl)-2-methyl-4-nitrobutanal

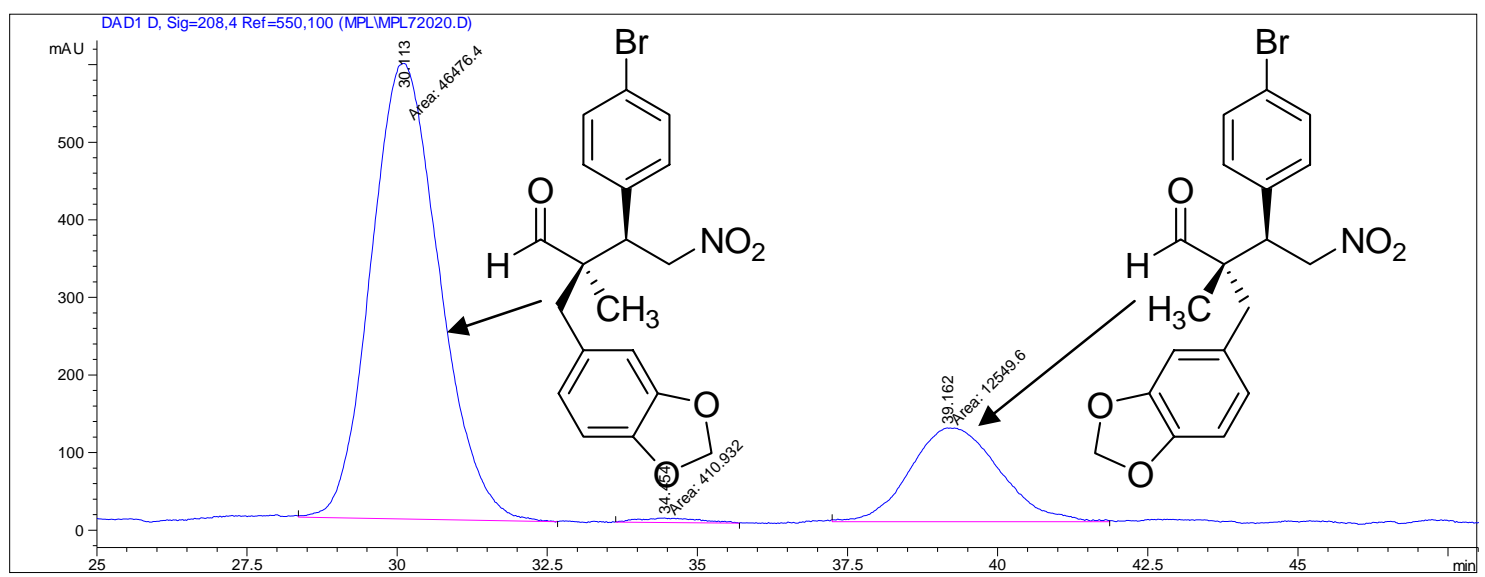

\begin{tabular}{|l|l|l|l|l|l|l|}
\hline $\begin{array}{l}\text { Peak } \\
\#\end{array}$ & $\begin{array}{l}\text { RetTime } \\
{[\mathrm{min}]}\end{array}$ & Type & $\begin{array}{l}\text { Width } \\
{[\mathrm{min}]}\end{array}$ & $\begin{array}{l}\text { Area } \\
{[\mathrm{mAU} * \mathrm{~s}]}\end{array}$ & $\begin{array}{l}\text { Height } \\
{[\mathrm{mAU}]}\end{array}$ & $\begin{array}{l}\text { Area } \\
{[\%]}\end{array}$ \\
\hline 1 & 30.113 & MM & 1.3197 & $4.64822 \mathrm{e} 4$ & 587.03113 & 78.7708 \\
\hline 2 & 34.454 & MM & 1.0085 & 288.76703 & 4.77232 & 0.4894 \\
\hline 3 & 39.162 & MM & 1.7001 & $1.22385 \mathrm{e} 4$ & 119.98075 & 20.7399 \\
\hline Totals: & & & & $5.90095 \mathrm{e} 4$ & 711.78420 & \\
\hline
\end{tabular}

(R)-2,2-dimethyl-4-nitro-3-phenylbutanal: General procedure, chromatography on silica (15\% diethyl ether/hexanes), colorless to light yellow liquid, $89 \%$ yield $(197.6 \mathrm{mg})$, $99 \%$ ee as determined by SFC (Chiralcel OD-H, 3.0\% methanol $/ \mathrm{CO}_{2}, 3.0 \mathrm{~mL} / \mathrm{min}, 208$ $\mathrm{nm}, 30{ }^{\circ} \mathrm{C} ; t_{r}$ (major enantiomer $)=4.37 \mathrm{~min}, t_{r}($ minor enantiomer $\left.)=5.29 \mathrm{~min}\right) .[\alpha]^{25}{ }_{\mathrm{D}}=$ $+8.7\left(\mathrm{c}=0.0163 \mathrm{~g} / 2.0 \mathrm{~mL}\right.$, chloroform). ${ }^{1} \mathrm{H}$ NMR $\left(400 \mathrm{MHz}, \mathrm{CDCl}_{3}\right): \delta 9.53(1 \mathrm{H}, \mathrm{s})$, $7.31(3 \mathrm{H}, \mathrm{m}), 7.13(2 \mathrm{H}, \mathrm{m}), 4.85(1 \mathrm{H}, \mathrm{dd}, \mathrm{J}=11.3,13.2 \mathrm{~Hz}), 4.69(1 \mathrm{H}, \mathrm{dd}, \mathrm{J}=4.4,13.2$ $\mathrm{Hz}), 3.78(1 \mathrm{H}, \mathrm{dd}, \mathrm{J}=4.4,11.3 \mathrm{~Hz}), 1.31(3 \mathrm{H}, \mathrm{s}), 1.00(3 \mathrm{H}, \mathrm{s}) .{ }^{13} \mathrm{C}\left(100 \mathrm{MHz}, \mathrm{CDCl}_{3}\right): \delta$ 204.2, 135.3, 129.0, 128.7, 128.1, 76.3, 48.4, 48.2, 21.6, 18.8. IR (neat): 3033 (w), 2974 (m), 2933 (w), 2819 (w), 2721 (w), 1725 (s), 1555 (s), 1496 (m), 1455 (m), 1379 (m), $882(\mathrm{~m}), 750(\mathrm{~m}), 705(\mathrm{~m})$. HRMS (ESI): expected for $\left[\mathrm{C}_{12} \mathrm{H}_{15} \mathrm{NO}_{3}+\mathrm{NH}_{4}\right]^{+}: 239.1396$, found: 239.1401 . 


\section{Racemic 2,2-dimethyl-4-nitro-3-phenylbutanal}

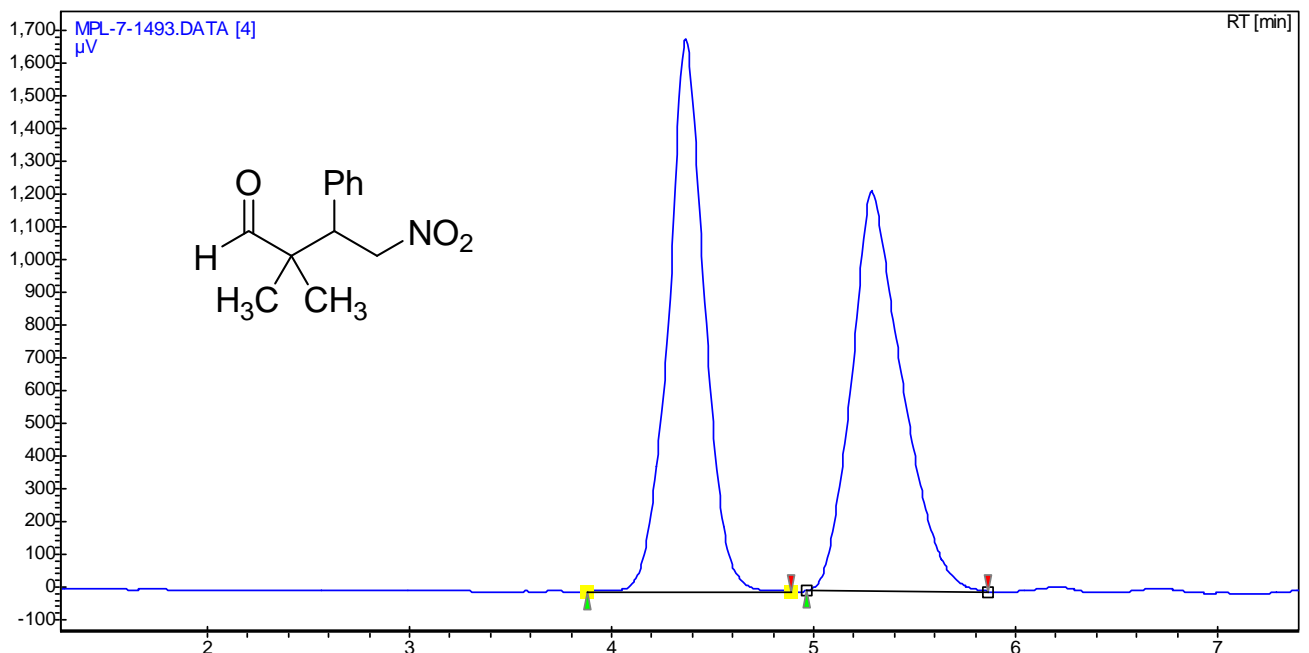

\begin{tabular}{|l|l|l|l|l|}
\hline$\#$ & Time $[\mathrm{Min}]$ & Height $[\mu \mathrm{V}]$ & Area $[\mu \mathrm{V} . \mathrm{Min}]$ & Area $[\%]$ \\
\hline 1 & 4.37 & 1687.3 & 350.3 & 49.591 \\
\hline 2 & 5.29 & 1222.5 & 356.1 & 50.409 \\
\hline & & & & \\
\hline Total & & 2909.9 & 706.3 & 100.000 \\
\hline
\end{tabular}

\section{Enantioenriched $(R)$-2,2-dimethyl-4-nitro-3-phenylbutanal}

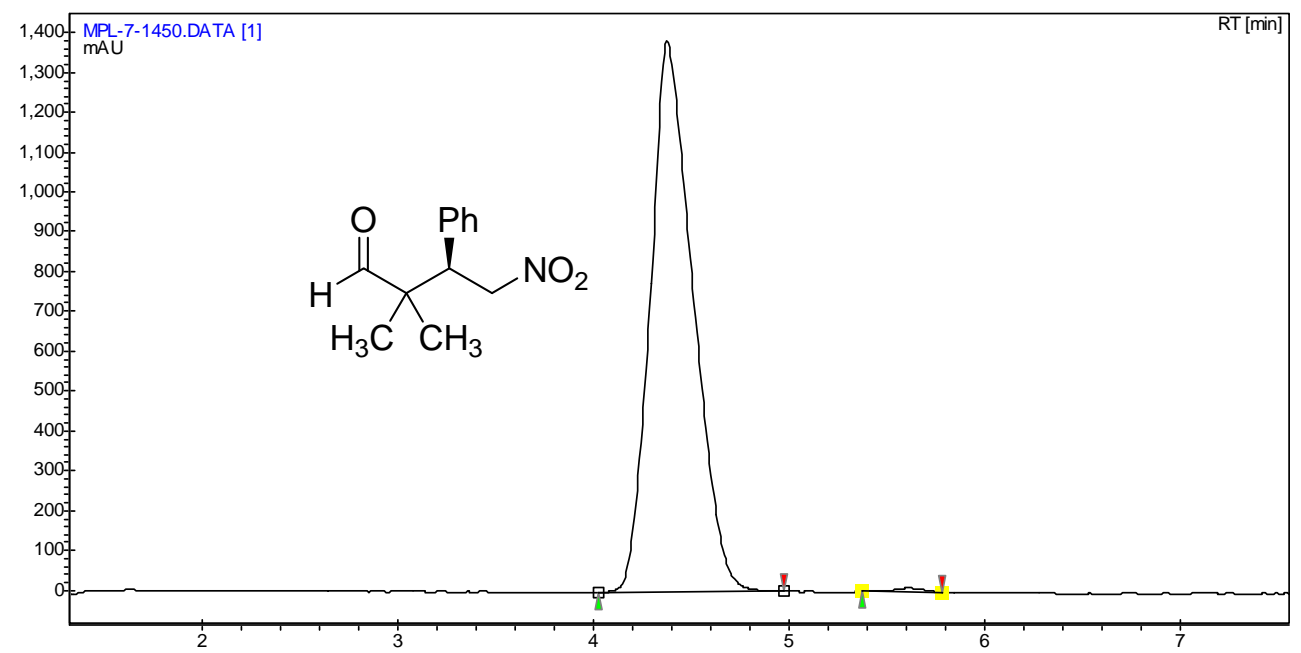

\begin{tabular}{|l|l|l|l|l|}
\hline$\#$ & Time $[\mathrm{Min}]$ & Height [mAU] & Area [mAU*min] & Area [\%] \\
\hline 1 & 4.37 & 1381.47 & 349.28 & 99.490 \\
\hline 2 & 5.61 & 9.74 & 1.79 & 0.510 \\
\hline & & & & \\
\hline Total & & & 351.07 & 100.000 \\
\hline
\end{tabular}


(2S,3R)-2-methyl-4-nitro-2,3-diphenylbutanal: General procedure, diastereomeric products separable by chromatography on silica (15\% diethyl ether/hexanes), white solid, m.p. $=76-78{ }^{\circ} \mathrm{C}, 94 \%$ yield $(267.7 \mathrm{mg}), 11.9: 1 \mathrm{dr}, 97 \%$ ee (major diastereomer) as determined by HPLC (Chiralcel OD-H, 5.0\% isopropanol/hexanes, $1.0 \mathrm{~mL} / \mathrm{min}, 208 \mathrm{~nm}$; $t_{r}($ minor enantiomer, major diastereomer $\left.)=22.77 \mathrm{~min}\right), t_{r}($ major enantiomer, major diastereomer $)=31.97 \mathrm{~min}) .[\alpha]^{25}=+170.9(\mathrm{c}=0.0227 \mathrm{~g} / 2.0 \mathrm{~mL}$, chloroform $) .{ }^{1} \mathrm{H}$ NMR $\left(400 \mathrm{MHz}, \mathrm{CDCl}_{3}\right): \delta 9.56(1 \mathrm{H}, \mathrm{s}), 7.24(3 \mathrm{H}, \mathrm{m}), 7.14(3 \mathrm{H}, \mathrm{m}), 7.06(2 \mathrm{H}, \mathrm{m}), 6.94(2 \mathrm{H}$, m), $5.03(1 \mathrm{H}, \mathrm{dd}, \mathrm{J}=11.7,13.2 \mathrm{~Hz}), 4.86(1 \mathrm{H}, \mathrm{dd}, \mathrm{J}=4.0,13.2 \mathrm{~Hz}), 4.20(1 \mathrm{H}, \mathrm{dd}, \mathrm{J}=4.0$, $11.7 \mathrm{~Hz}), 1.53(3 \mathrm{H}, \mathrm{s}) .{ }^{13} \mathrm{C}\left(100 \mathrm{MHz}, \mathrm{CDCl}_{3}\right): \delta 201.0,137.3,135.4,129.3,129.0,128.2$, 128.1, 127.7, 127.3, 76.2, 56.6, 49.6, 16.8. IR (thin film): $3062(\mathrm{~m}), 3032(\mathrm{~m}), 2977$ (m), $2923(\mathrm{~m}), 2822(\mathrm{~m}), 2719(\mathrm{~m}), 1957$ (w), 1885 (w), 1809 (w), 1721 (s), 1562 (s), 1495 (m), $1454(\mathrm{~m}), 1377(\mathrm{~s}), 1216(\mathrm{~m}), 1096(\mathrm{~m}), 1030(\mathrm{~m}), 918(\mathrm{~m}), 858(\mathrm{~m}), 759(\mathrm{~s}), 700$ (s). HRMS (CI): expected for $\left[\mathrm{C}_{17} \mathrm{H}_{17} \mathrm{NO}_{3}+\mathrm{NH}_{4}\right]^{+}: 301.1552$, found: 301.1549 .

(2R,3R)-2-methyl-4-nitro-2,3-diphenylbutanal: colorless to light yellow oil, $32 \%$ ee (minor diastereomer) as determined by HPLC (Chiralcel OD-H, $5.0 \%$ isopropanol/hexanes, $1.0 \mathrm{~mL} / \mathrm{min}, 208 \mathrm{~nm} ; t_{r}$ (major enantiomer, minor diastereomer) $=$ $20.48 \mathrm{~min}, t_{r}($ minor enantiomer, minor diastereomer $)=27.44 \mathrm{~min} .[\alpha]^{25}{ }_{\mathrm{D}}=-16.4(\mathrm{c}=$ $0.0078 \mathrm{~g} / 2.0 \mathrm{~mL}$, chloroform). ${ }^{1} \mathrm{H}$ NMR $\left(400 \mathrm{MHz}, \mathrm{CDCl}_{3}\right): \delta 9.57(1 \mathrm{H}, \mathrm{s}), 7.43(3 \mathrm{H}, \mathrm{m})$, $7.25(3 \mathrm{H}, \mathrm{m}), 7.11(2 \mathrm{H}, \mathrm{m}), 6.97(2 \mathrm{H}, \mathrm{m}), 4.68(1 \mathrm{H}, \mathrm{dd}, \mathrm{J}=3.7,12.8 \mathrm{~Hz}), 4.51(1 \mathrm{H}, \mathrm{dd}, \mathrm{J}$ $=11.7,12.8 \mathrm{~Hz}), 4.37(1 \mathrm{H}, \mathrm{dd}, \mathrm{J}=3.7,11.7 \mathrm{~Hz}), 1.39(3 \mathrm{H}, \mathrm{s}) .{ }^{13} \mathrm{C}\left(100 \mathrm{MHz}, \mathrm{CDCl}_{3}\right): \delta$ 200.0, 135.6, 135.0, 129.6, 129.1, 128.5, 128.3, 128.1, 128.0, 76.5, 56.2, 47.8, 17.8. IR (neat): $3063(\mathrm{~m}), 3033(\mathrm{~m}), 2975(\mathrm{~m}), 2921(\mathrm{~m}), 2820(\mathrm{~m}), 2719(\mathrm{~m}), 1960(\mathrm{w}), 1889$ (w), 1811 (w), 1724 (s), 1552 (s), 1497 (m), 1454 (m), 1378 (s), 1205 (m), 1093 (m), 906 (m), 702 (s). HRMS (ESI): expected for $\left[\mathrm{C}_{17} \mathrm{H}_{17} \mathrm{NO}_{3}+\mathrm{NH}_{4}\right]^{+}: 301.1552$, found: 301.1560 . 


\section{Racemic 2-methyl-4-nitro-2,3-diphenylbutanal}

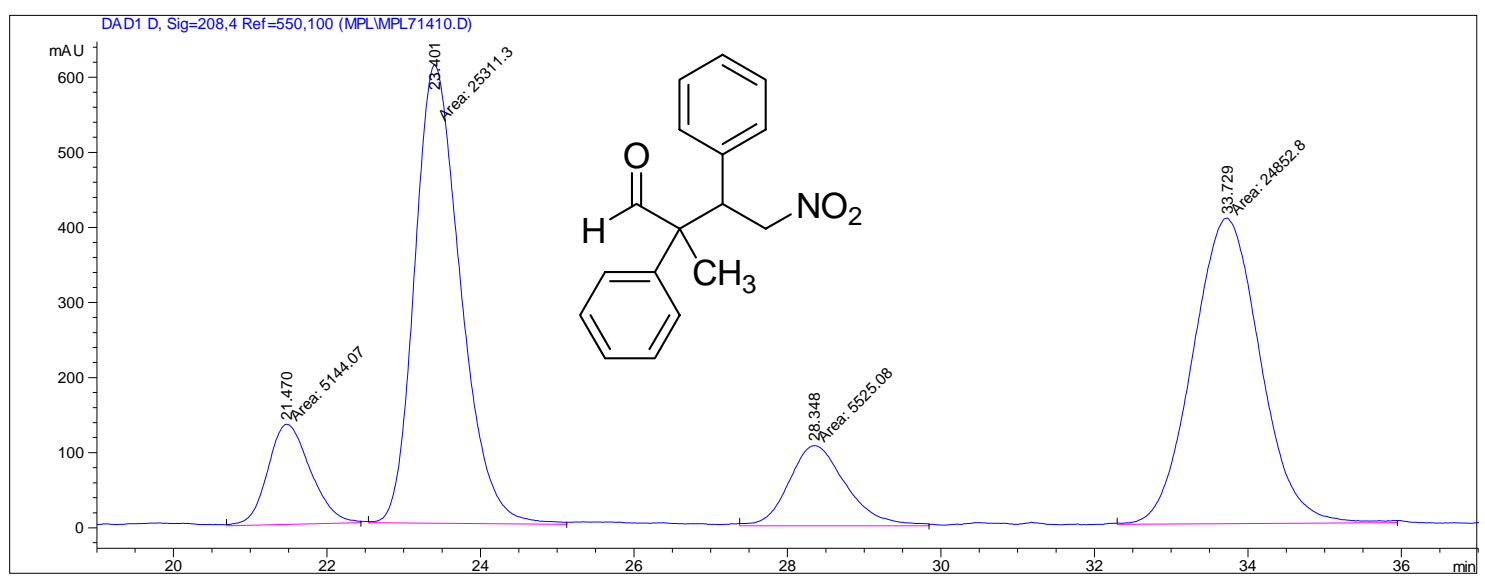

\begin{tabular}{|l|l|l|l|l|l|l|}
\hline $\begin{array}{l}\text { Peak } \\
\#\end{array}$ & $\begin{array}{l}\text { RetTime } \\
{[\mathrm{min}]}\end{array}$ & Type & $\begin{array}{l}\text { Width } \\
{[\mathrm{min}]}\end{array}$ & $\begin{array}{l}\text { Area } \\
{[\mathrm{mAU} \text { s }]}\end{array}$ & $\begin{array}{l}\text { Height } \\
{[\mathrm{mAU}]}\end{array}$ & $\begin{array}{l}\text { Area } \\
{[\%]}\end{array}$ \\
\hline 1 & 21.470 & $\mathrm{MM}$ & 0.6502 & 5218.46484 & 133.76399 & 8.6173 \\
\hline 2 & 23.401 & $\mathrm{MM}$ & 0.6855 & $2.50737 \mathrm{e} 4$ & 609.59735 & 41.4042 \\
\hline 3 & 28.348 & $\mathrm{MM}$ & 0.8327 & 5227.61572 & 104.63694 & 8.6324 \\
\hline 4 & 33.729 & $\mathrm{MM}$ & 1.0242 & $2.50385 \mathrm{e} 4$ & 407.46124 & 41.3461 \\
\hline Totals: & & & & $6.05582 \mathrm{e} 4$ & 1255.45953 & \\
\hline
\end{tabular}

Enantioenriched (2S,3R)-2-methyl-4-nitro-2,3-diphenylbutanal + (2R,3R)-2-methyl4-nitro-2,3-diphenylbutanal

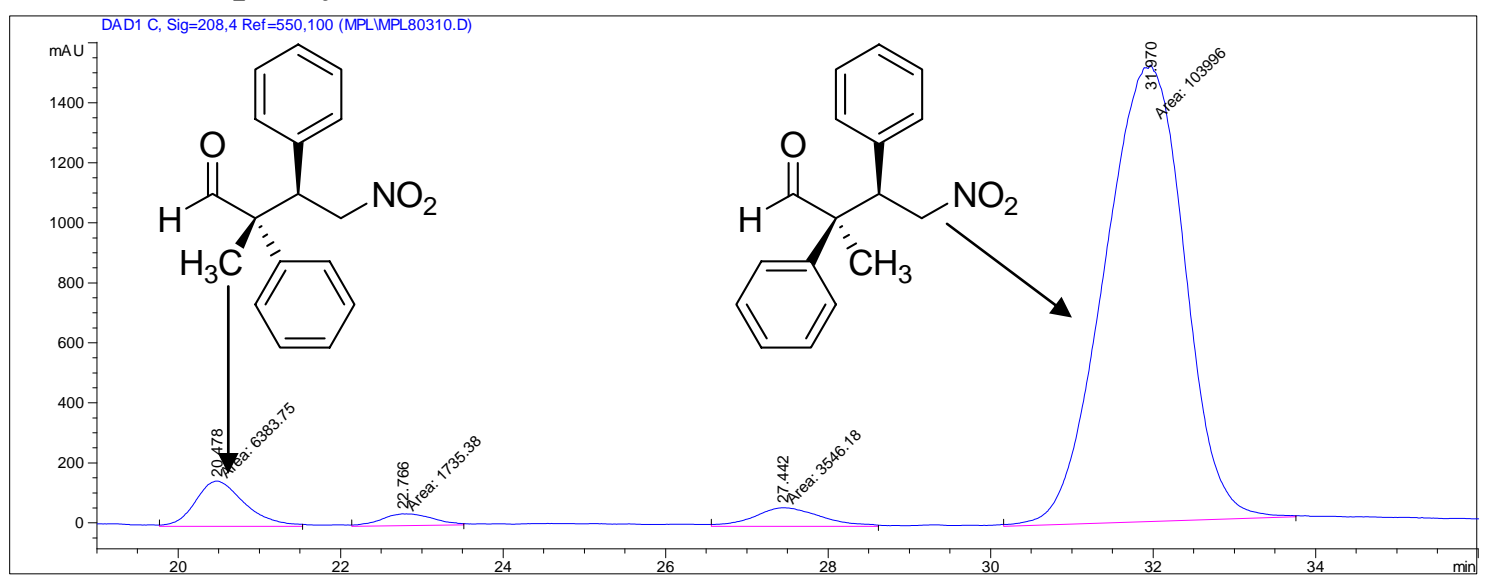

\begin{tabular}{|l|l|l|l|l|l|l|}
\hline $\begin{array}{l}\text { Peak } \\
\#\end{array}$ & $\begin{array}{l}\text { RetTime } \\
{[\mathrm{min}]}\end{array}$ & Type & $\begin{array}{l}\text { Width } \\
{[\mathrm{min}]}\end{array}$ & $\begin{array}{l}\text { Area } \\
{\left[\mathrm{mAU}^{*} \mathrm{~s}\right]}\end{array}$ & $\begin{array}{l}\text { Height } \\
{[\mathrm{mAU}]}\end{array}$ & $\begin{array}{l}\text { Area } \\
{[\%]}\end{array}$ \\
\hline 1 & 20.478 & MM & 0.6718 & 5880.19775 & 145.87737 & 5.1001 \\
\hline 2 & 22.766 & MM & 0.6898 & 1475.95178 & 35.65924 & 1.2801 \\
\hline 3 & 27.442 & MM & 0.8372 & 2833.51196 & 56.40998 & 2.4576 \\
\hline 4 & 31.970 & MM & 1.1491 & $1.05107 \mathrm{e} 5$ & 1524.43115 & 91.1622 \\
\hline
\end{tabular}


Totals:

$1.15297 \mathrm{e} 5$

1762.37774

General Procedure for the Synthesis of Primary Amine-Thiourea Catalysts.

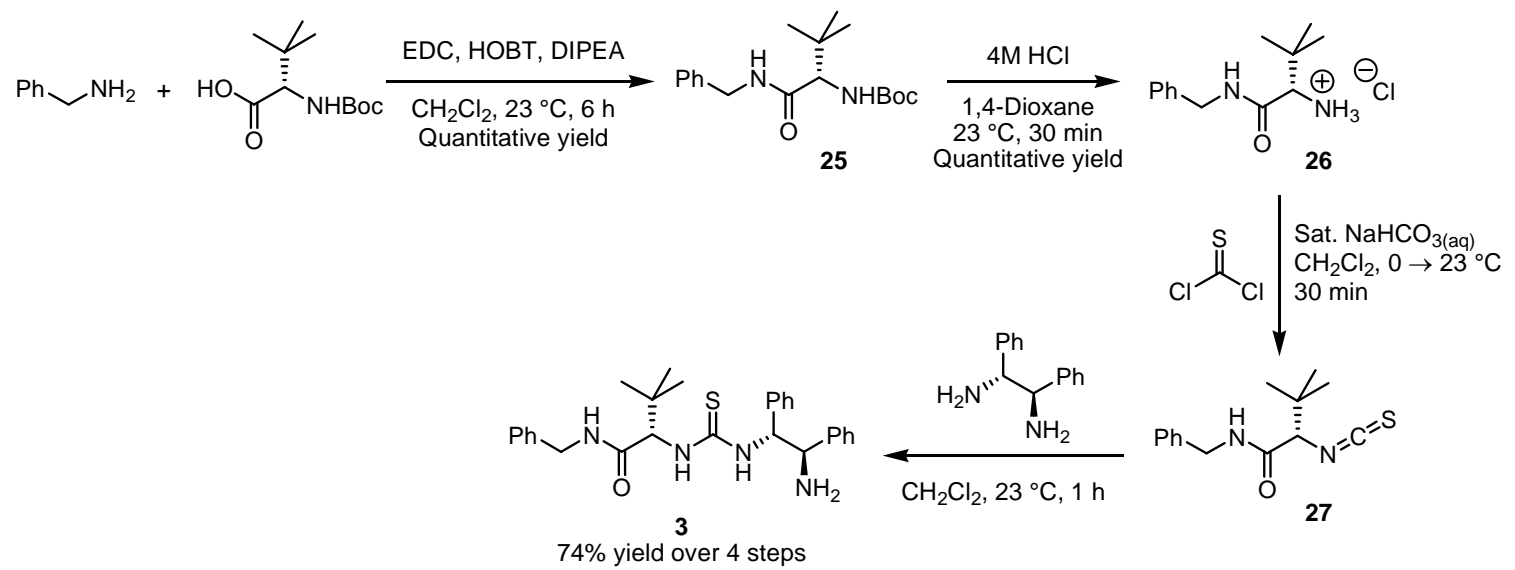

(S)-2-(3-((1R,2R)-2-amino-1,2-diphenylethyl)thioureido)- $N$-benzyl-3,3-

dimethylbutanamide (3): $N$-Boc-L-tert-leucine (1.00 g, $4.32 \mathrm{mmol}, 1.00$ equiv.), 1-(3dimethylaminopropyl)-3-ethylcarbodiimide hydrochloride $(912.5 \mathrm{mg}, 4.76 \mathrm{mmol}, 1.10$ equiv.) and 1-hydroxybenzotriazole hydrate $(643.2 \mathrm{mg}, 4.76 \mathrm{mmol}, 1.10$ equiv.) were loaded into an oven-dried round-bottomed flask equipped with a magnetic stir bar, rubber septum, and nitrogen inlet. The solids were dissolved in dichloromethane $(21.6 \mathrm{~mL})$ and the resulting clear colorless solution was stirred $(\sim 500 \mathrm{rpm})$ at room temperature. Benzylamine (520 $\mu \mathrm{L}, 4.76 \mathrm{mmol}, 1.10$ equiv.) and $N, N$-diisopropylethylamine $(829 \mu \mathrm{L}$, $4.76 \mathrm{mmol}, 1.10$ equiv.) were sequentially added and the reaction mixture was stirred at room temperature for 6 hours. The reaction mixture was poured into a separatory funnel containing a $1 \mathrm{M} \mathrm{HCl}$ solution $(20.0 \mathrm{~mL})$. The phases were separated and the aqueous phase was extracted with dichloromethane $(20.0 \mathrm{~mL})$. The organic phases were combined, washed sequentially with saturated aqueous sodium bicarbonate and brine, dried over sodium sulfate, filtered, and concentrated under reduced pressure to provide amide 25 as a white solid in quantitative yield. A magnetic stir bar and $4 \mathrm{M} \mathrm{HCl}$ in 1,4dioxane $(9.7 \mathrm{~mL}, 38.9 \mathrm{mmol}, 9.0$ equiv.) were added to a round-bottomed flask containing 25 and the reaction mixture was stirred for 30 minutes at room temperature. The magnetic stir bar was removed, the solvent was evaporated under reduced pressure, and the resulting residue was dried in vacuo to provide $\mathbf{2 6}$ in quantitative yield as a white foamy solid. A magnetic stir bar, dichloromethane $(43.2 \mathrm{~mL})$, and saturated aqueous sodium bicarbonate $(43.2 \mathrm{~mL}$ ) were added to a flask containing 26 and the resulting clear colorless solution was cooled to $0{ }^{\circ} \mathrm{C}$ with an ice bath. Thiophosgene $(346 \mu \mathrm{L}, 4.54$ mmol, 1.05 equiv.) was added via syringe and the resulting light orange biphasic mixture was stirred vigorously at $0{ }^{\circ} \mathrm{C}$ for 10 minutes, warmed to room temperature, and stirred at room temperature for 20 minutes. The biphasic mixture was poured into a separatory funnel and the phases were separated. The aqueous phase was extracted with dichloromethane $(40.0 \mathrm{~mL})$ and the organic phases were combined, washed with brine, dried over sodium sulfate, filtered, and concentrated under reduced pressure. The resulting yellow residue was dissolved in dichloromethane $(43.2 \mathrm{~mL}),(R, R)-1,2-$ diphenylethylenediamine ( $318.4 \mathrm{mg}, 6.48 \mathrm{mmol}, 1.50$ equiv.) was added in one portion, 
and the yellow reaction mixture was stirred at room temperature for 1.0 hour. The solvent was evaporated and the resulting yellow residue was purified by chromatography on silica (3.0\% methanol/dichloromethane). The title compound was obtained as a white foamy solid, m.p. $=102{ }^{\circ} \mathrm{C}$, in $74 \%$ yield $(1.520 \mathrm{~g})$ over four steps, $[\alpha]^{25}{ }_{\mathrm{D}}=27.9(\mathrm{c}=$ $0.0216 \mathrm{~g} / 2.0 \mathrm{~mL}$, chloroform). ${ }^{1} \mathrm{H} \mathrm{NMR}\left(400 \mathrm{MHz}, \mathrm{CDCl}_{3}\right): \delta 7.58(1 \mathrm{H}$, br s), 7.31-7.15 $(15 \mathrm{H}, \mathrm{m}), 6.82(1 \mathrm{H}$, br s), $6.31(1 \mathrm{H}$, br s), $5.00(1 \mathrm{H}$, br s $), 4.68(1 \mathrm{H}$, br s $), 4.37(1 \mathrm{H}, \mathrm{dd}, \mathrm{J}$ $=5.9,14.6 \mathrm{~Hz}), 4.24-4.20(2 \mathrm{H}, \mathrm{m}), 1.84(2 \mathrm{H}, \mathrm{br} \mathrm{s}), 0.95(9 \mathrm{H}, \mathrm{s}) .{ }^{13} \mathrm{C}\left(100 \mathrm{MHz}, \mathrm{CDCl}_{3}\right)$ : $\delta 182.8,170.6,141.6,139.3,137.7,128.6,128.5,128.3,127.7,127.4,127.2,126.8,66.3$, 64.7, 60.7, 43.3, 34.7, 26.9. IR (thin film): 3294 (s), 3062 (m), 3031 (m), 2964 (m), 2872 (m), 1951 (w), $1878(\mathrm{w}), 1808(\mathrm{w}), 1645$ (s), 1531 (s), $1454(\mathrm{~m}), 1357(\mathrm{~m}), 1233(\mathrm{~m})$, $1070(\mathrm{~m}), 1029$ (m), 755 (s), 699 (s), 667 (m), 593(m). LRMS (ESI): 475.2 (100\%) $\left[\mathrm{C}_{28} \mathrm{H}_{34} \mathrm{~N}_{4} \mathrm{OS}+\mathrm{H}\right]^{+}$.

(S)-2-(3-((1R,2R)-2-amino-1,2-diphenylethyl)thioureido)- $N$-benzyl-3-

methylbutanamide (23): General procedure, chromatography on silica $(3.0 \%$ methanol/dichloromethane), white foamy solid, m.p. $=169^{\circ} \mathrm{C}, 68 \%$ yield $(1.353 \mathrm{~g})$ over four steps, $[\alpha]_{\mathrm{D}}^{25}=18.7$ (c = $0.0211 \mathrm{~g} / 2.0 \mathrm{~mL}$, chloroform). ${ }^{1} \mathrm{H}$ NMR (400 MHz, $\left.\mathrm{CDCl}_{3}\right): \delta 7.71(1 \mathrm{H}$, br s$), 7.28-7.17(16 \mathrm{H}, \mathrm{m}), 6.52(1 \mathrm{H}$, br s $), 5.21(1 \mathrm{H}, \mathrm{br} \mathrm{s}), 4.73(1 \mathrm{H}$, br s), $4.42(1 \mathrm{H}, \mathrm{dd}, \mathrm{J}=5.9,14.6 \mathrm{~Hz}), 4.29-4.24(2 \mathrm{H}, \mathrm{m}), 2.20(1 \mathrm{H}, \mathrm{m}), 1.82(1 \mathrm{H}, \mathrm{br} \mathrm{s})$, $0.92-0.86(6 \mathrm{H}, \mathrm{m}) .{ }^{13} \mathrm{C}\left(100 \mathrm{MHz}, \mathrm{CDCl}_{3}\right): \delta 182.8,171.8,141.7,139.6,137.6,128.5$, $128.3,127.4,127.3,126.9,126.7,64.2,60.6,43.3,30.6,19.1,18.5$. IR (thin film): 3292 (s), $3063(\mathrm{~m}), 3030(\mathrm{~m}), 2963(\mathrm{~m}), 2930(\mathrm{~m}), 2873(\mathrm{~m}), 1955(\mathrm{w}), 1888(\mathrm{w}), 1810(\mathrm{w})$, 1651 (s), 1537 (s), 1453 (m), 1356 (m), 1259 (m), 1073 (m), 1029 (m), 755 (s), 698 (s), 605 (m). LRMS (ESI): $461.2(100 \%)\left[\mathrm{C}_{27} \mathrm{H}_{32} \mathrm{~N}_{4} \mathrm{OS}+\mathrm{H}\right]^{+}$.

(S)-2-(3-((1S,2S)-2-amino-1,2-diphenylethyl)thioureido)- $N$-benzyl-3-

methylbutanamide (24): General procedure, chromatography on silica $(3.0 \%$ methanol/dichloromethane), white foamy solid, m.p. $=87-88{ }^{\circ} \mathrm{C}, 69 \%$ yield $(1.373 \mathrm{~g})$ over four steps, $[\alpha]^{25}=-51.5\left(\mathrm{c}=0.0201 \mathrm{~g} / 2.0 \mathrm{~mL}\right.$, chloroform). ${ }^{1} \mathrm{H}$ NMR (400 MHz, $\left.\mathrm{CDCl}_{3}\right): \delta 7.67(1 \mathrm{H}$, br s), 7.34-7.19 $(16 \mathrm{H}, \mathrm{m}), 6.62(1 \mathrm{H}$, br s), $4.68(1 \mathrm{H}$, br s), $4.45(1 \mathrm{H}$, $\mathrm{dd}, \mathrm{J}=5.9,14.6 \mathrm{~Hz}), 4.32(1 \mathrm{H}, \mathrm{dd}, \mathrm{J}=5.9,14.6 \mathrm{~Hz}), 4.24$ (1H, br s), 2.04 (1H, br s), 1.51 $(2 \mathrm{H}$, br s $), 0.76\left(3 \mathrm{H}\right.$, br s), $0.65(3 \mathrm{H}$, br s $) .{ }^{13} \mathrm{C}\left(100 \mathrm{MHz}, \mathrm{CDCl}_{3}\right): \delta 182.3,171.9,141.8$, 139.6, 137.6, 128.6, 128.5, 128.2, 127.3, 127.2, 126.8, 126.6, 64.0, 63.5, 60.3, 43.2, 30.8, 19.0, 18.2. IR (thin film): 3291 (s), 3063 (m), 3030 (m), 2962 (m), 2931 (m), $2874(\mathrm{~m})$, 1952 (w), 1879 (w), 1810 (w), 1651 (s), 1536 (s), 1453 (m), 1358 (m), 1265 (m), 736 (m), 699 (s), 598 (m). LRMS (ESI): $461.2(100 \%)\left[\mathrm{C}_{27} \mathrm{H}_{32} \mathrm{~N}_{4} \mathrm{OS}+\mathrm{H}\right]^{+}$.

1-((1S,2S)-2-aminocyclohexyl)-3-((R)-3,3-dimethylbutan-2-yl)thiourea (4): Saturated aqueous sodium bicarbonate $(25.0 \mathrm{~mL})$ and dichloromethane $(25.0 \mathrm{~mL})$ were loaded into a $200 \mathrm{~mL}$ round-bottomed flask equipped with a magnetic stir bar. $(R)$-3,3-dimethyl-2butylamine ( $250.0 \mathrm{mg}, 2.47 \mathrm{mmol}, 1.0$ equiv.) was added via syringe and the biphasic mixture was cooled to $0{ }^{\circ} \mathrm{C}$ with an ice bath. Thiophosgene $(198.0 \mu \mathrm{L}, 2.59 \mathrm{mmol}, 1.05$ equiv.) was added and the biphasic mixture was stirred vigorously for 10.0 minutes at 0 ${ }^{\circ} \mathrm{C}$, warmed to room temperature, and stirred for an additional 20.0 minutes. The biphasic mixture was poured into a separatory funnel and the organic phase was separated. The aqueous layer was washed with dichloromethane $(25.0 \mathrm{~mL})$, the organic phases were 
combined, washed with brine, dried over sodium sulfate, filtered, and concentrated. The yellow residue was dissolved in dichloromethane and a magnetic stir bar was added to the solution. (S,S)-1,2-diaminocyclohexane $(564.0 \mathrm{mg}, 4.94 \mathrm{mmol}, 2.0$ equiv.) was added in one portion and the solution was stirred at room temperature for 16 hours. The solvent was evaporated and the yellow residue was purified by chromatography on silica $(2.0 \%$ $\mathrm{CH}_{3} \mathrm{OH}, 1.0 \% \mathrm{Et}_{3} \mathrm{~N}, 97 \% \mathrm{CH}_{2} \mathrm{Cl}_{2}$ ). The title compound was obtained as a white solid (m.p. $\left.=113-115^{\circ} \mathrm{C}\right)$ in $92 \%$ yield $(587.3 \mathrm{mg}),[\alpha]^{25}{ }_{\mathrm{D}}=-133.0(\mathrm{c}=0.0200 \mathrm{~g} / 2.0 \mathrm{~mL}$, chloroform). ${ }^{1} \mathrm{H}$ NMR $\left(400 \mathrm{MHz}, \mathrm{CDCl}_{3}\right): \delta 6.12(1 \mathrm{H}, \mathrm{br} \mathrm{s}), 4.15(2 \mathrm{H}, \mathrm{br} \mathrm{s}), 3.23(1 \mathrm{H}, \mathrm{br}$ s), $2.09(2 \mathrm{H}$, br m), $1.96(1 \mathrm{H}$, br m), $1.83(1 \mathrm{H}$, br m), $1.71(2 \mathrm{H}$, br m), $1.22(4 \mathrm{H}$, br m), $1.10(3 \mathrm{H}, \mathrm{d}, \mathrm{J}=6.6 \mathrm{~Hz}), 0.92(9 \mathrm{H}, \mathrm{s}) \cdot{ }^{13} \mathrm{C}\left(100 \mathrm{MHz}, \mathrm{CDCl}_{3}\right): \delta 182.5,61.9,59.3$, 56.2, 34.5, 34.3, 32.1, 26.5, 24.8, 24.6, 15.3. IR (thin film): $3259(\mathrm{~m}), 3058(\mathrm{~m}), 2962(\mathrm{~s})$, $2935(\mathrm{~s}), 2859(\mathrm{~m}), 2209(\mathrm{w}), 1541(\mathrm{~s}), 1365(\mathrm{~m}), 1240(\mathrm{~m}), 1200(\mathrm{~m}), 1087(\mathrm{~m}), 915$ (m), 732 (s). LRMS (CI): $223.4(100 \%)\left[\mathrm{C}_{13} \mathrm{H}_{27} \mathrm{~N}_{3} \mathrm{~S}-\mathrm{H}_{2} \mathrm{~S}\right]^{+}$.

1-((1R,2R)-2-aminocyclohexyl)-3-((R)-3,3-dimethylbutan-2-yl)thiourea

(28): Procedure identical to that utilized for the synthesis of $\mathbf{4}$ with the exception that $(R, R)$ 1,2-diaminocyclohexane is used instead of $(S, S)$-1,2-diaminocyclohexane. Chromatography on silica $\left(2.0 \% \mathrm{CH}_{3} \mathrm{OH}, 1.0 \% \mathrm{Et}_{3} \mathrm{~N}, 97 \% \mathrm{CH}_{2} \mathrm{Cl}_{2}\right)$, white solid (m.p. $=$ $\left.174-175{ }^{\circ} \mathrm{C}\right), 91 \%$ yield $(578.7 \mathrm{mg}) .[\alpha]^{25}{ }_{\mathrm{D}}=+79.5(\mathrm{c}=0.0201 \mathrm{~g} / 2.0 \mathrm{~mL}$, chloroform). ${ }^{1} \mathrm{H}$ NMR $\left(400 \mathrm{MHz}, \mathrm{CDCl}_{3}\right): \delta 6.39(1 \mathrm{H}, \mathrm{br} \mathrm{s}), 4.24(2 \mathrm{H}, \mathrm{br} \mathrm{s}), 3.24(1 \mathrm{H}, \mathrm{br} \mathrm{s}), 2.53(2 \mathrm{H}$, $\mathrm{m}), 1.97(1 \mathrm{H}, \mathrm{br} \mathrm{m}), 1.81(1 \mathrm{H}, \mathrm{br} \mathrm{m}), 1.66(4 \mathrm{H}, \mathrm{br} \mathrm{m}), 1.18(4 \mathrm{H}, \mathrm{br} \mathrm{m}), 1.06(3 \mathrm{H}, \mathrm{d}, \mathrm{J}=$ $6.6 \mathrm{~Hz}), 0.90(9 \mathrm{H}, \mathrm{s}) .{ }^{13} \mathrm{C}\left(100 \mathrm{MHz}, \mathrm{CDCl}_{3}\right): \delta 182.0,61.8,59.0,55.9,34.6,34.4,32.1$, 26.4, 24.7, 24.6, 16.1. IR (thin film): 3260 (m), 3057 (m), 2963 (s), 2934 (s), $2859(\mathrm{~m})$, $2210(\mathrm{w}), 1542(\mathrm{~s}), 1448(\mathrm{~m}), 1365(\mathrm{~m}), 1241(\mathrm{~m}), 1201(\mathrm{~m}), 1085(\mathrm{~m}), 914(\mathrm{~m}), 732(\mathrm{~s})$. LRMS (CI): $223.4(100 \%)\left[\mathrm{C}_{13} \mathrm{H}_{27} \mathrm{~N}_{3} \mathrm{~S}-\mathrm{H}_{2} \mathrm{~S}\right]^{+}$. 

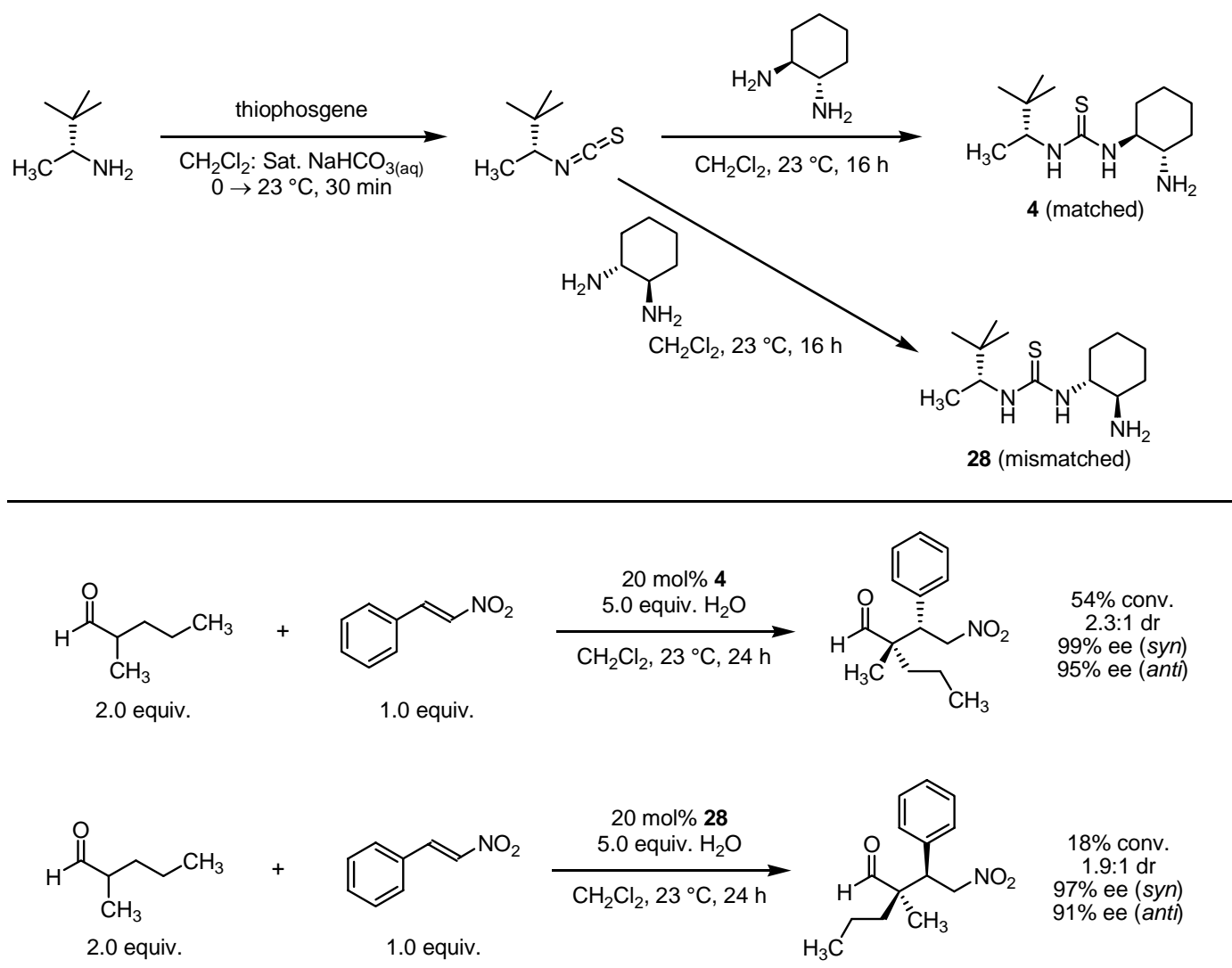

\section{General Procedure for the Synthesis of Racemic Samples.}

2,3-dimethyl-4-nitro-2-phenylbutanal: 2-Phenylpropionaldehyde (332 $\mu \mathrm{L}, 2.5 \mathrm{mmol}$, 10.0 equiv.) is loaded into a $1 / 2$ dram vial equipped with a magnetic stir bar. Thiocarbanilide (11.4 mg, $0.05 \mathrm{mmol}, 20 \mathrm{~mol} \%$ ), pyrrolidine $(4.2 \mu \mathrm{L}, 0.05 \mathrm{mmol}, 20$ mol\%), and 1-nitroprop-1-ene ( $87.1 \mathrm{mg}, 0.25 \mathrm{mmol}, 1.0$ equiv.) are sequentially added. The vial is capped, and the yellow mixture is stirred at room temperature for 24 hours. The resulting orange mixture is loaded directly onto a silica gel column ( $8 \%$ diethyl ether/hexanes), providing the title compound as a colorless to light yellow liquid in $92 \%$ yield $(51.0 \mathrm{mg})$ with a 20:1 diastereomeric ratio as measured by SFC (Chiralcel OD-H, $2.0 \%$ methanol $/ \mathrm{CO}_{2}, 2.0 \mathrm{~mL} / \mathrm{min}, 208 \mathrm{~nm}, 30{ }^{\circ} \mathrm{C}$; $t_{r}$ (minor diastereomer) $=6.73 \mathrm{~min}$, $t_{r}($ minor diastereomer $)=6.73 \mathrm{~min}, t_{r}($ major diastereomer $\left.)=7.44 \mathrm{~min}\right), t_{r}($ major diastereomer) $=8.25 \mathrm{~min}$ ).

\section{Probing Catalyst Deactivation}

Incubation of catalyst and aldehyde for 12 hours prior to nitroalkene addition had no observable detrimental effect on product yield. Interestingly, aldol addition or condensation products were not observed or isolated upon stirring catalyst and aldehyde for 12 hours. Alternatively, incubation of catalyst and nitroalkene for 12 hours prior to aldehyde addition resulted in lower product yield. Primary amine-catalyzed nitroalkene polymerization, rather than catalyst deactivation, was identified as the origin of lower product yield under these circumstances. The excess of aldehyde required under optimized conditions may prevent nitroalkene decomposition or polymerization by ensuring that the catalyst rests in the imine state. 
Careful monitoring of the reaction of 2-phenylpriopionaldehyde with 1-nitrohex-1-ene catalyzed by 1 ( $20 \mathrm{~mol} \%)$ revealed that substrate conversion ceased after 24 hours, and that both starting materials could be recovered alongside product. These observations suggested catalyst deactivation either by decomposition or poisoning pathways. Addition of a second $20 \mathrm{~mol} \%$ aliquot of catalyst to a stalled reaction mixture, followed by further stirring for 24 hours, led to complete conversion and generation of 5 in $92 \%$ isolated yield, indicating that build-up of catalyst poisons was not responsible for the deactivation. Similar yields were obtained in the presence, or absence, of a full equivalent of added 5, thus ruling out product inhibition.

It was therefore postulated that decomposition of an intermediate along the catalytic cycle was responsible for catalyst deactivation. A zwitterionic intermediate is often invoked in the numerous studies concerning the mechanism of conjugate addition of enamines to nitroalkenes. ${ }^{[6]}$ This intermediate may undergo hydrolysis to a nitroaldehyde, or collapse to a 1,2-oxazine-N-oxide or cyclobutane. 1,2-Oxazine-N-oxides resulting from the addition of aldehyde-derived enamines to nitroalkenes have been shown to hydrolyze readily upon exposure to atmospheric moisture. ${ }^{[6]]}$ Conversely, cyclobutanes resulting from the addition of aldehyde-derived enamines to nitroalkenes only undergo hydrolysis when treated with aqueous acid (such as $1 \mathrm{M} \mathrm{HCl)} .^{[6,1]}$

[6] a) K. C. Brannock, A. Bell, R. D. Burpitt, C. A. Kelly, J. Org. Chem. 1964, 29, 801-812; b) M. E. Kuehne, L. Foley, J. Org. Chem. 1965, 30, 4280-4284; c) A. Risaliti, S. Fatutta, M. Forchiassin, E. Valentin, Tetrahedron Lett. 1966, 7, 1821-1825; d) A. Risaliti, M. Forchiassin, E. Valentin, Tetrahedron Lett. 1966, 7, 6331-6335; e) A. T. Nielsen, T. G. Archibald, Tetrahedron 1970, 26, 3475-3485; f) D. Seebach, J. Golinski, Helv. Chim. Acta 1981, 64, 1413-1423; g) S. J. Blarer, W. B. Schweizer, D. Seebach, Helv. Chim. Acta 1982, 65, 1637-1654; h) S. J. Blarer, D. Seebach, Chem. Ber. 1983, 116, 3086-3096; i) D. Seebach, A. K. Beck, J. Golinski, J. N. Hay, T. Laube, Helv. Chim. Acta 1985, 68, 162-172; j) F. Felluga, P. Nitti, G. Pitacco, E. Valentin, Tetrahedron 1989, 45, 2099-2108; k) F. Felluga, P. Nitti, G. Pitacco, E. Valentin, Tetrahedron 1989, 45, 5667-5678; 1) F. Felluga, P. Nitti, G. Pitacco, E. Valentin, J. Chem. Soc., Perkin Trans. 1 1992, 2331-2335. 


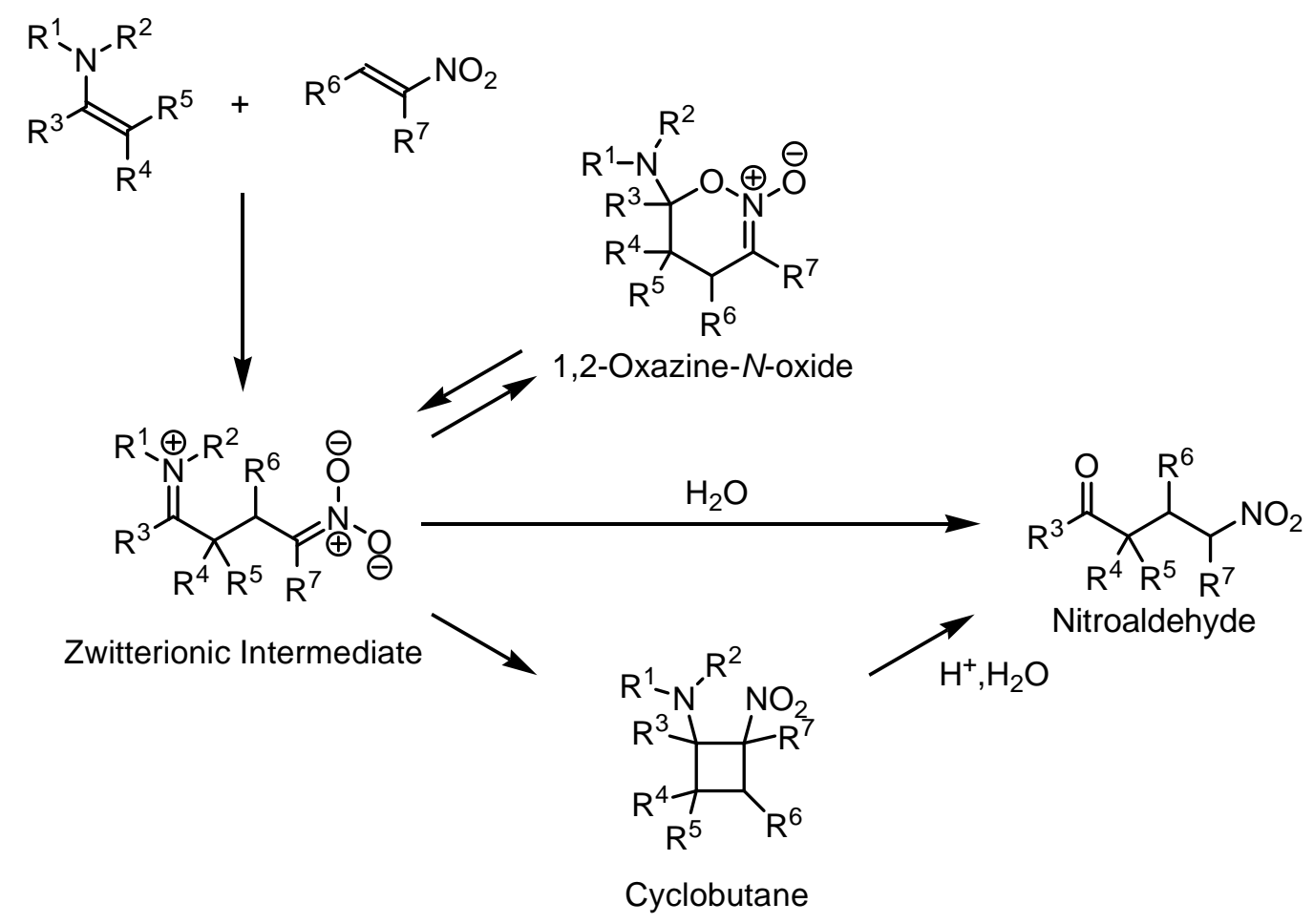

The beneficial role of water is most likely due to acceleration of imine hydrolysis and/or 1,2-oxazine-N-oxide decomposition. However, a cyclobutane intermediate is unlikely to undergo hydrolysis under the non-acidic reaction conditions, and its formation could therefore lead to catalyst deactivation. If cyclobutane formation is responsible for catalyst deactivation, the acidic workup $(1 \mathrm{M} \mathrm{HCl})$ would release an amount of product roughly equivalent to the catalyst loading. Indeed, replacing the $\mathrm{HCl}$ workup with a simple aqueous workup in the addition of 2-phenylpropionaldehyde to 1-nitrohex-1-ene reduced the isolated yield of product from $54 \%$ to $39 \%$. This corresponds closely to the catalyst loading $(20 \mathrm{~mol} \%)$. In addition, the only byproduct that could be isolated from the reaction that was not treated with $1 \mathrm{M} \mathrm{HCl}$ possessed mass spectral characteristics consistent with the expected cyclobutane intermediate (HRMS-ESI $(\mathrm{M}+\mathrm{H})^{+}$Calcd: 622.3785; Found: 622.3786). NMR analysis of this byproduct was precluded by extensive peak broadening due to hydrogen bonding. Zwitterionic, imine, and 1,2oxazine- $N$-oxide intermediates are most likely unstable under the aqueous reaction conditions or silica gel chromatography. Hence, the results above lend credence to the hypothesis that catalyst deactivation occurs through irreversible cyclobutane formation. Unfortunately, no acidic additives could be identified that were both capable of cyclobutane hydrolysis and compatible with the catalytic conditions.

\section{Structural Data for compound 22:}

\section{Experimental Section:}

X-Ray quality crystals were obtained via slow evaporation of a dichloromethane 
solution of 22. A colorless block crystal with dimensions $0.20 \times 0.16 \times 0.12 \mathrm{~mm}$ was mounted on a glass fiber using a very small amount of paratone oil.

Data were collected using a Bruker SMART CCD (charge coupled device) based diffractometer equipped with an Oxford Cryostream low-temperature apparatus operating at $193 \mathrm{~K}$. Data were measured using omega scans of $0.3^{\circ}$ per frame for 30 seconds, such that a hemisphere was collected. A total of 1271 frames were collected with a maximum resolution of $0.76 \AA$. The first 50 frames were recollected at the end of data collection to monitor for decay. Cell parameters were retrieved using SMART ${ }^{1}$ software and refined using SAINT on all observed reflections. Data reduction was performed using the SAINT software ${ }^{2}$ which corrects for Lp and decay. Absorption corrections were applied using SADABS ${ }^{6}$ multiscan technique, supplied by George Sheldrick. The structures are solved by the direct method using the SHELXS $-97^{3}$ program and refined by least squares method on $\mathrm{F}^{2}$, SHELXL-97, ${ }^{4}$ incorporated in SHELXTL-PC V 6.10.

The structure was solved in the space group P $2_{1} 2_{1} 2_{1}$ (\# 19) by analysis of systematic absences. All non-hydrogen atoms are refined anisotropically. Hydrogens were calculated by geometrical methods and refined as a riding model. The Flack ${ }^{7}$ parameter is used to determine chirality of the crystal studied, the value should be near zero, a value of one is the other enantiomer and a value of 0.5 is racemic. The Flack parameter was refined to $0.028(7)$, confirming the absolute stereochemistry. The crystal used for the diffraction study showed no decomposition during data collection. All drawing are done at $50 \%$ ellipsoids.

Acknowledgement. The CCD based x-ray diffractometer at Harvard University was purchased through NIH grant (1S10RR11937-01).

\section{References}

1. SMART V 5.625 (NT) Software for the CCD Detector System; Bruker Analytical X-ray Systems, Madison, WI (2001).

2. SAINT V 6.22 (NT) Software for the CCD Detector System Bruker Analytical Xray Systems, Madison, WI (2001).

3. Sheldrick, G. M. SHELXS-90, Program for the Solution of Crystal Structure, University of Göttingen, Germany, 1990.

4. Sheldrick, G. M. SHELXL-97, Program for the Refinement of Crystal Structure, University of Göttingen, Germany, 1997.

5. SHELXTL 6.1 (PC-Version), Program library for Structure Solution and Molecular Graphics; Bruker Analytical X-ray Systems, Madison, WI (2000).

6. SADABS. Program for absorption corrections using Siemens CCD based on the method of Robert Blessing; Blessing, R.H. Acta Cryst. A51 1995, 33-38.

7. Flack, H. D.. Acta Cryst. A39, 1983, 876-881. 
${ }^{a}$ Obtained with graphite monochromated Mo K $\alpha(\lambda=0.71073 \AA)$ radiation. ${ }^{\mathrm{b}} R 1=\sum|| F_{\mathrm{o}}|-| F_{\mathrm{c}}|| / \sum\left|F_{\mathrm{o}}\right| .{ }^{\mathrm{c}} w R_{2}=\left\{\sum\left[w\left(F_{\mathrm{o}}{ }^{2}-F_{\mathrm{c}}{ }^{2}\right)^{2} /\left\{\sum\left[w\left(F_{\mathrm{o}}{ }^{2}\right)^{2}\right]\right\}^{1 / 2}\right.\right.$.

The following are 50\% thermal ellipsoidal drawings of the molecule in the asymmetric cell with various amount of labeling. 


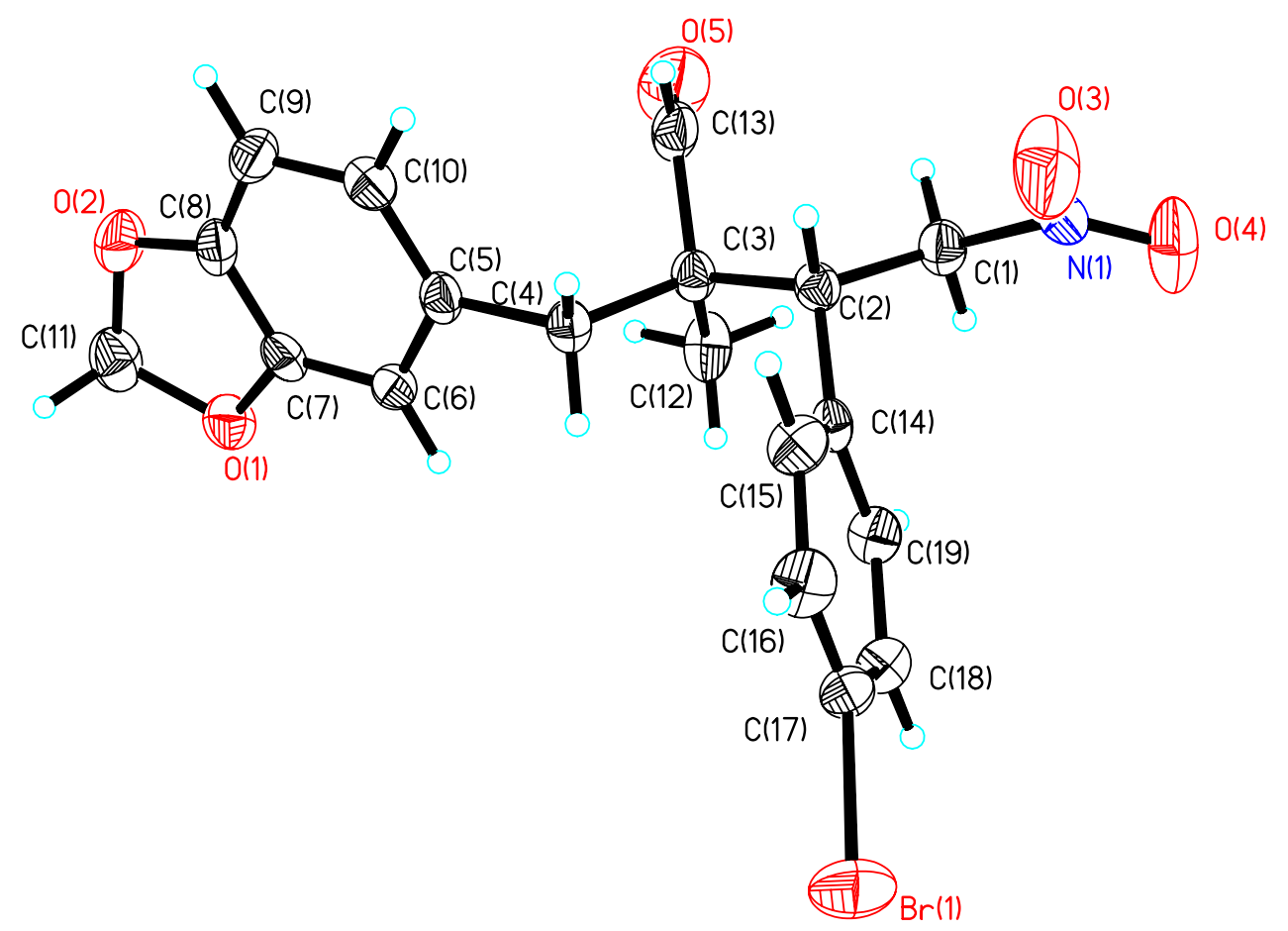




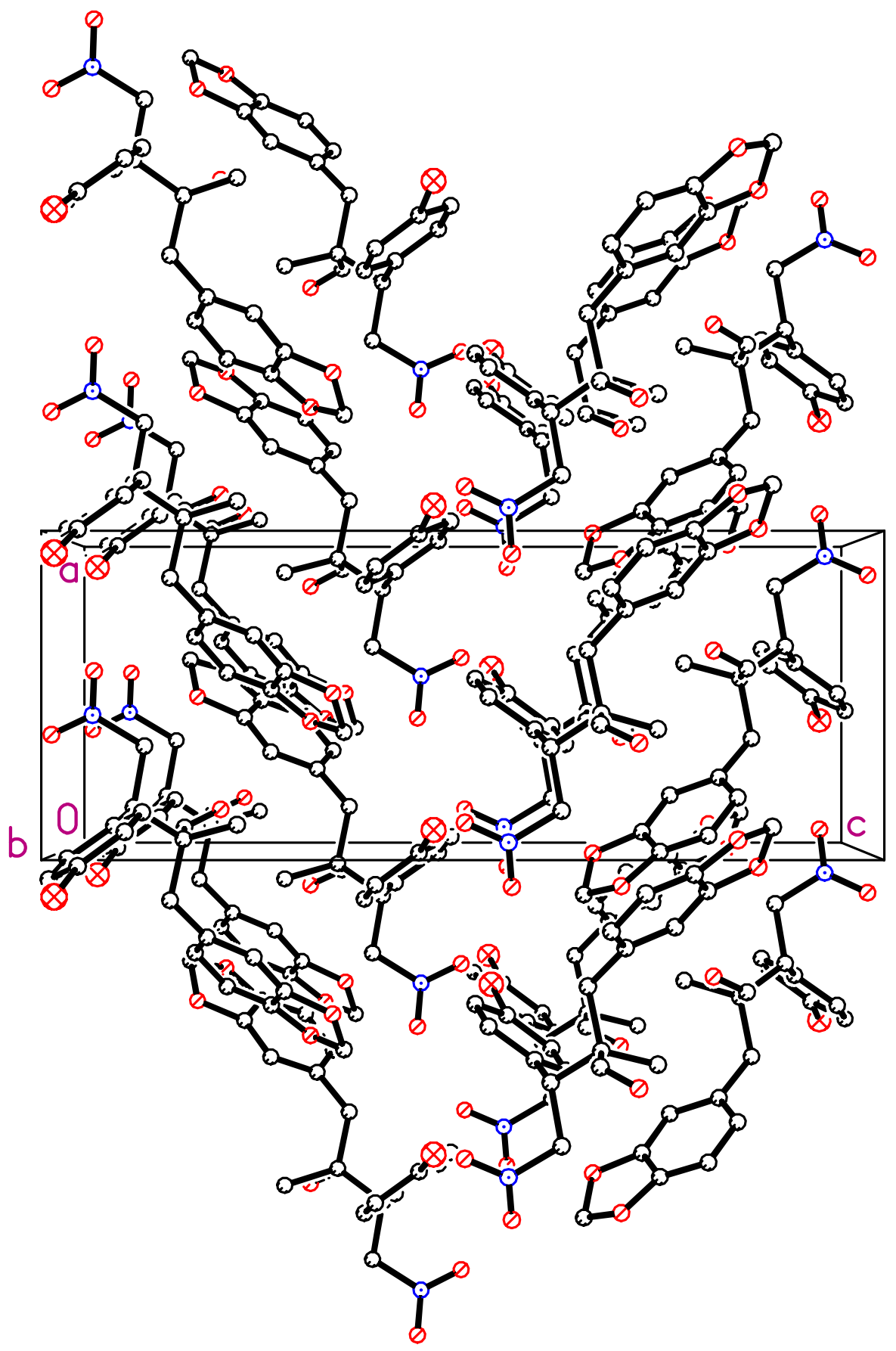

This is a drawing of the packing along the b-axis. 
Table 1. Crystal data and structure refinement for 22.

Identification code

Empirical formula

Formula weight

Temperature

Wavelength

Crystal system

Space group

Unit cell dimensions

Volume

Z

Density (calculated)

Absorption coefficient

$\mathrm{F}(000)$

Crystal size

Theta range for data collection

Index ranges

Reflections collected

Independent reflections

Completeness to theta $=27.88^{\circ}$

Absorption correction

Max. and min. transmission

Refinement method

Data / restraints / parameters

Goodness-of-fit on $\mathrm{F}^{2}$

Final $\mathrm{R}$ indices $[\mathrm{I}>2 \operatorname{sigma}(\mathrm{I})]$

$\mathrm{R}$ indices (all data)

Absolute structure parameter

Largest diff. peak and hole enj72t

C19 H18 Br N O5

420.25

193(2) K

$0.71073 \AA$

Orthorhombic

P2(1)2(1)2(1)

$\mathrm{a}=8.0316(9) \AA$ $\alpha=90^{\circ}$.

$\mathrm{b}=10.9639(12) \AA$ $\beta=90^{\circ}$.

$\mathrm{c}=20.583(2) \AA$ $\gamma=90^{\circ}$.

1812.5(3) $\AA^{3}$

4

$1.540 \mathrm{Mg} / \mathrm{m}^{3}$

$2.298 \mathrm{~mm}^{-1}$

856

$0.20 \times 0.16 \times 0.12 \mathrm{~mm}^{3}$

1.98 to $27.88^{\circ}$.

$-10<=\mathrm{h}<=10,-14<=\mathrm{k}<=14,-27<=\mathrm{l}<=15$

11673

$4263[\mathrm{R}($ int $)=0.0267]$

$99.6 \%$

Empirical

0.7700 and 0.6565

Full-matrix least-squares on $\mathrm{F}^{2}$

4263 / 0 / 236

0.980

$\mathrm{R} 1=0.0334, \mathrm{wR} 2=0.0631$

$\mathrm{R} 1=0.0483, \mathrm{wR} 2=0.0668$

$0.028(7)$

0.401 and -0.338 e. $\AA^{-3}$ 
Table 2. Atomic coordinates $\left(\mathrm{x} 10^{4}\right)$ and equivalent isotropic displacement parameters $\left(\AA^{2} \times 10^{3}\right)$ for 22. $U(e q)$ is defined as one third of the trace of the orthogonalized $U^{i j}$ tensor.

\begin{tabular}{|c|c|c|c|c|}
\hline & $\mathrm{x}$ & $\mathrm{y}$ & $\mathrm{z}$ & $\mathrm{U}(\mathrm{eq})$ \\
\hline $\operatorname{Br}(1)$ & 9149(1) & $13687(1)$ & $354(1)$ & $51(1)$ \\
\hline $\mathrm{O}(1)$ & $5057(2)$ & $7624(2)$ & $3411(1)$ & $37(1)$ \\
\hline $\mathrm{O}(2)$ & $4164(2)$ & $5699(2)$ & $3104(1)$ & $40(1)$ \\
\hline $\mathrm{O}(3)$ & $13783(3)$ & $8242(2)$ & 23(1) & $79(1)$ \\
\hline $\mathrm{O}(4)$ & $15743(2)$ & $9030(2)$ & $576(1)$ & $58(1)$ \\
\hline $\mathrm{O}(5)$ & 11371(3) & 5921(2) & 1993(1) & $67(1)$ \\
\hline $\mathrm{N}(1)$ & $14389(2)$ & $8550(2)$ & $534(1)$ & $35(1)$ \\
\hline $\mathrm{C}(1)$ & $13426(3)$ & $8362(2)$ & 1153(1) & $30(1)$ \\
\hline$C(2)$ & $11540(3)$ & $8468(2)$ & $1025(1)$ & $24(1)$ \\
\hline $\mathrm{C}(3)$ & 10533(2) & 7905(2) & 1605(1) & $26(1)$ \\
\hline$C(4)$ & $8645(2)$ & $8132(2)$ & $1453(1)$ & $26(1)$ \\
\hline$C(5)$ & 7439(3) & 7444(2) & 1877(1) & $26(1)$ \\
\hline$C(6)$ & $6865(3)$ & $7975(2)$ & 2461(1) & $27(1)$ \\
\hline$C(7)$ & 5743(3) & $7315(2)$ & $2816(1)$ & $27(1)$ \\
\hline$C(8)$ & $5188(3)$ & $6176(2)$ & $2629(1)$ & $30(1)$ \\
\hline$C(9)$ & $5715(3)$ & $5638(2)$ & $2065(1)$ & $33(1)$ \\
\hline$C(10)$ & $6855(3)$ & $6309(2)$ & $1690(1)$ & $31(1)$ \\
\hline$C(11)$ & $3838(3)$ & $6688(2)$ & $3536(1)$ & $42(1)$ \\
\hline$C(12)$ & $11002(3)$ & $8407(2)$ & $2272(1)$ & $40(1)$ \\
\hline$C(13)$ & $10830(3)$ & $6539(2)$ & $1569(1)$ & $37(1)$ \\
\hline$C(14)$ & $11034(3)$ & $9760(2)$ & $852(1)$ & $24(1)$ \\
\hline$C(15)$ & $10048(3)$ & $9967(2)$ & $308(1)$ & $31(1)$ \\
\hline$C(16)$ & 9489(3) & $11135(2)$ & $150(1)$ & $35(1)$ \\
\hline $\mathrm{C}(17)$ & 9941(3) & $12089(2)$ & $539(1)$ & $30(1)$ \\
\hline$C(18)$ & $10956(3)$ & $11922(2)$ & $1076(1)$ & $31(1)$ \\
\hline$C(19)$ & $11485(3)$ & $10757(2)$ & $1226(1)$ & $29(1)$ \\
\hline
\end{tabular}


Table 3. Bond lengths $[\AA]$ and angles $\left[{ }^{\circ}\right]$ for 22.

\begin{tabular}{|c|c|c|c|}
\hline $\operatorname{Br}(1)-C(17)$ & $1.902(2)$ & $\mathrm{O}(1)-\mathrm{C}(7)$ & $1.383(3)$ \\
\hline $\mathrm{O}(1)-\mathrm{C}(11)$ & $1.443(3)$ & $\mathrm{O}(2)-\mathrm{C}(8)$ & $1.381(3)$ \\
\hline $\mathrm{O}(2)-\mathrm{C}(11)$ & $1.427(3)$ & $\mathrm{O}(3)-\mathrm{N}(1)$ & $1.208(3)$ \\
\hline $\mathrm{O}(4)-\mathrm{N}(1)$ & $1.211(3)$ & $\mathrm{O}(5)-\mathrm{C}(13)$ & $1.187(3)$ \\
\hline $\mathrm{N}(1)-\mathrm{C}(1)$ & $1.504(3)$ & $C(1)-C(2)$ & $1.542(3)$ \\
\hline $\mathrm{C}(1)-\mathrm{H}(1 \mathrm{~A})$ & 0.9900 & $\mathrm{C}(1)-\mathrm{H}(1 \mathrm{~B})$ & 0.9900 \\
\hline$C(2)-C(14)$ & $1.517(3)$ & $C(2)-C(3)$ & $1.568(3)$ \\
\hline $\mathrm{C}(2)-\mathrm{H}(2)$ & 1.0000 & $C(3)-C(13)$ & $1.518(3)$ \\
\hline$C(3)-C(12)$ & $1.527(3)$ & $C(3)-C(4)$ & $1.568(3)$ \\
\hline$C(4)-C(5)$ & $1.507(3)$ & $\mathrm{C}(4)-\mathrm{H}(4 \mathrm{~A})$ & 0.9900 \\
\hline C(4)-H(4B) & 0.9900 & $C(5)-C(10)$ & $1.384(3)$ \\
\hline$C(5)-C(6)$ & $1.412(3)$ & $C(6)-C(7)$ & $1.368(3)$ \\
\hline $\mathrm{C}(6)-\mathrm{H}(6)$ & 0.9500 & $C(7)-C(8)$ & $1.381(3)$ \\
\hline $\mathrm{C}(8)-\mathrm{C}(9)$ & $1.370(3)$ & $C(9)-C(10)$ & $1.405(3)$ \\
\hline $\mathrm{C}(9)-\mathrm{H}(9)$ & 0.9500 & $\mathrm{C}(10)-\mathrm{H}(10)$ & 0.9500 \\
\hline $\mathrm{C}(11)-\mathrm{H}(11 \mathrm{~A})$ & 0.9900 & $\mathrm{C}(11)-\mathrm{H}(11 \mathrm{~B})$ & 0.9900 \\
\hline $\mathrm{C}(12)-\mathrm{H}(12 \mathrm{~A})$ & 0.9800 & $\mathrm{C}(12)-\mathrm{H}(12 \mathrm{~B})$ & 0.9800 \\
\hline $\mathrm{C}(12)-\mathrm{H}(12 \mathrm{C})$ & 0.9800 & $\mathrm{C}(13)-\mathrm{H}(13)$ & 0.9500 \\
\hline$C(14)-C(19)$ & $1.385(3)$ & $\mathrm{C}(14)-\mathrm{C}(15)$ & $1.390(3)$ \\
\hline$C(15)-C(16)$ & $1.395(3)$ & $\mathrm{C}(15)-\mathrm{H}(15)$ & 0.9500 \\
\hline$C(16)-C(17)$ & $1.365(3)$ & $\mathrm{C}(16)-\mathrm{H}(16)$ & 0.9500 \\
\hline $\mathrm{C}(17)-\mathrm{C}(18)$ & $1.385(3)$ & $\mathrm{C}(18)-\mathrm{C}(19)$ & $1.382(3)$ \\
\hline $\mathrm{C}(18)-\mathrm{H}(18)$ & 0.9500 & $\mathrm{C}(19)-\mathrm{H}(19)$ & 0.9500 \\
\hline $\mathrm{C}(7)-\mathrm{O}(1)-\mathrm{C}(11)$ & $104.75(19)$ & $\mathrm{C}(8)-\mathrm{O}(2)-\mathrm{C}(11)$ & $105.25(18)$ \\
\hline $\mathrm{O}(3)-\mathrm{N}(1)-\mathrm{O}(4)$ & $123.1(2)$ & $\mathrm{O}(3)-\mathrm{N}(1)-\mathrm{C}(1)$ & $119.5(2)$ \\
\hline $\mathrm{O}(4)-\mathrm{N}(1)-\mathrm{C}(1)$ & $117.5(2)$ & $\mathrm{N}(1)-\mathrm{C}(1)-\mathrm{C}(2)$ & $110.51(19)$ \\
\hline $\mathrm{N}(1)-\mathrm{C}(1)-\mathrm{H}(1 \mathrm{~A})$ & 109.5 & $\mathrm{C}(2)-\mathrm{C}(1)-\mathrm{H}(1 \mathrm{~A})$ & 109.5 \\
\hline $\mathrm{N}(1)-\mathrm{C}(1)-\mathrm{H}(1 \mathrm{~B})$ & 109.5 & $\mathrm{C}(2)-\mathrm{C}(1)-\mathrm{H}(1 \mathrm{~B})$ & 109.5 \\
\hline $\mathrm{H}(1 \mathrm{~A})-\mathrm{C}(1)-\mathrm{H}(1 \mathrm{~B})$ & 108.1 & $\mathrm{C}(14)-\mathrm{C}(2)-\mathrm{C}(1)$ & $111.97(18)$ \\
\hline $\mathrm{C}(14)-\mathrm{C}(2)-\mathrm{C}(3)$ & $114.08(17)$ & $\mathrm{C}(1)-\mathrm{C}(2)-\mathrm{C}(3)$ & $110.30(18)$ \\
\hline $\mathrm{C}(14)-\mathrm{C}(2)-\mathrm{H}(2)$ & 106.7 & $\mathrm{C}(1)-\mathrm{C}(2)-\mathrm{H}(2)$ & 106.7 \\
\hline $\mathrm{C}(3)-\mathrm{C}(2)-\mathrm{H}(2)$ & 106.7 & $\mathrm{C}(13)-\mathrm{C}(3)-\mathrm{C}(12)$ & $111.1(2)$ \\
\hline$C(13)-C(3)-C(4)$ & $107.38(18)$ & $\mathrm{C}(12)-\mathrm{C}(3)-\mathrm{C}(4)$ & $111.12(19)$ \\
\hline
\end{tabular}




\begin{tabular}{|c|c|c|c|}
\hline$C(13)-C(3)-C(2)$ & $105.66(18)$ & $C(12)-C(3)-C(2)$ & $114.59(18)$ \\
\hline $\mathrm{C}(4)-\mathrm{C}(3)-\mathrm{C}(2)$ & $106.55(17)$ & $C(5)-C(4)-C(3)$ & $115.30(18)$ \\
\hline $\mathrm{C}(5)-\mathrm{C}(4)-\mathrm{H}(4 \mathrm{~A})$ & 108.4 & $\mathrm{C}(3)-\mathrm{C}(4)-\mathrm{H}(4 \mathrm{~A})$ & 108.4 \\
\hline $\mathrm{C}(5)-\mathrm{C}(4)-\mathrm{H}(4 \mathrm{~B})$ & 108.4 & $\mathrm{C}(3)-\mathrm{C}(4)-\mathrm{H}(4 \mathrm{~B})$ & 108.4 \\
\hline $\mathrm{H}(4 \mathrm{~A})-\mathrm{C}(4)-\mathrm{H}(4 \mathrm{~B})$ & 107.5 & $\mathrm{C}(10)-\mathrm{C}(5)-\mathrm{C}(6)$ & $119.8(2)$ \\
\hline $\mathrm{C}(10)-\mathrm{C}(5)-\mathrm{C}(4)$ & $120.5(2)$ & $C(6)-C(5)-C(4)$ & $119.7(2)$ \\
\hline $\mathrm{C}(7)-\mathrm{C}(6)-\mathrm{C}(5)$ & $116.8(2)$ & $\mathrm{C}(7)-\mathrm{C}(6)-\mathrm{H}(6)$ & 121.6 \\
\hline $\mathrm{C}(5)-\mathrm{C}(6)-\mathrm{H}(6)$ & 121.6 & $\mathrm{C}(6)-\mathrm{C}(7)-\mathrm{C}(8)$ & $122.8(2)$ \\
\hline$C(6)-C(7)-O(1)$ & $127.3(2)$ & $\mathrm{C}(8)-\mathrm{C}(7)-\mathrm{O}(1)$ & $109.88(19)$ \\
\hline $\mathrm{C}(9)-\mathrm{C}(8)-\mathrm{C}(7)$ & $121.8(2)$ & $\mathrm{C}(9)-\mathrm{C}(8)-\mathrm{O}(2)$ & $128.4(2)$ \\
\hline $\mathrm{C}(7)-\mathrm{C}(8)-\mathrm{O}(2)$ & $109.7(2)$ & $\mathrm{C}(8)-\mathrm{C}(9)-\mathrm{C}(10)$ & $116.1(2)$ \\
\hline $\mathrm{C}(8)-\mathrm{C}(9)-\mathrm{H}(9)$ & 121.9 & $\mathrm{C}(10)-\mathrm{C}(9)-\mathrm{H}(9)$ & 121.9 \\
\hline $\mathrm{C}(5)-\mathrm{C}(10)-\mathrm{C}(9)$ & $122.7(2)$ & $\mathrm{C}(5)-\mathrm{C}(10)-\mathrm{H}(10)$ & 118.7 \\
\hline $\mathrm{C}(9)-\mathrm{C}(10)-\mathrm{H}(10)$ & 118.7 & $\mathrm{O}(2)-\mathrm{C}(11)-\mathrm{O}(1)$ & 107.71(19) \\
\hline $\mathrm{O}(2)-\mathrm{C}(11)-\mathrm{H}(11 \mathrm{~A})$ & 110.2 & $\mathrm{O}(1)-\mathrm{C}(11)-\mathrm{H}(11 \mathrm{~A})$ & 110.2 \\
\hline $\mathrm{O}(2)-\mathrm{C}(11)-\mathrm{H}(11 \mathrm{~B})$ & 110.2 & $\mathrm{O}(1)-\mathrm{C}(11)-\mathrm{H}(11 \mathrm{~B})$ & 110.2 \\
\hline $\mathrm{H}(11 \mathrm{~A})-\mathrm{C}(11)-\mathrm{H}(11 \mathrm{~B})$ & 108.5 & $\mathrm{C}(3)-\mathrm{C}(12)-\mathrm{H}(12 \mathrm{~A})$ & 109.5 \\
\hline $\mathrm{C}(3)-\mathrm{C}(12)-\mathrm{H}(12 \mathrm{~B})$ & 109.5 & $\mathrm{H}(12 \mathrm{~A})-\mathrm{C}(12)-\mathrm{H}(12 \mathrm{~B})$ & 109.5 \\
\hline $\mathrm{C}(3)-\mathrm{C}(12)-\mathrm{H}(12 \mathrm{C})$ & 109.5 & $\mathrm{H}(12 \mathrm{~A})-\mathrm{C}(12)-\mathrm{H}(12 \mathrm{C})$ & 109.5 \\
\hline $\mathrm{H}(12 \mathrm{~B})-\mathrm{C}(12)-\mathrm{H}(12 \mathrm{C})$ & 109.5 & $\mathrm{O}(5)-\mathrm{C}(13)-\mathrm{C}(3)$ & $125.8(3)$ \\
\hline $\mathrm{O}(5)-\mathrm{C}(13)-\mathrm{H}(13)$ & 117.1 & $\mathrm{C}(3)-\mathrm{C}(13)-\mathrm{H}(13)$ & 117.1 \\
\hline$C(19)-C(14)-C(15)$ & $117.9(2)$ & $C(19)-C(14)-C(2)$ & $122.4(2)$ \\
\hline$C(15)-C(14)-C(2)$ & $119.66(19)$ & $C(14)-C(15)-C(16)$ & $121.3(2)$ \\
\hline $\mathrm{C}(14)-\mathrm{C}(15)-\mathrm{H}(15)$ & 119.3 & $\mathrm{C}(16)-\mathrm{C}(15)-\mathrm{H}(15)$ & 119.3 \\
\hline$C(17)-C(16)-C(15)$ & $118.8(2)$ & $\mathrm{C}(17)-\mathrm{C}(16)-\mathrm{H}(16)$ & 120.6 \\
\hline$C(15)-C(16)-H(16)$ & 120.6 & $C(16)-C(17)-C(18)$ & $121.5(2)$ \\
\hline$C(16)-C(17)-B r(1)$ & $119.97(18)$ & $\mathrm{C}(18)-\mathrm{C}(17)-\mathrm{Br}(1)$ & $118.53(17)$ \\
\hline$C(19)-C(18)-C(17)$ & $118.8(2)$ & $\mathrm{C}(19)-\mathrm{C}(18)-\mathrm{H}(18)$ & 120.6 \\
\hline $\mathrm{C}(17)-\mathrm{C}(18)-\mathrm{H}(18)$ & 120.6 & $C(18)-C(19)-C(14)$ & $121.6(2)$ \\
\hline $\mathrm{C}(18)-\mathrm{C}(19)-\mathrm{H}(19)$ & 119.2 & $\mathrm{C}(14)-\mathrm{C}(19)-\mathrm{H}(19)$ & 119.2 \\
\hline
\end{tabular}

Symmetry transformations used to generate equivalent atoms: 
Table 4. Anisotropic displacement parameters $\left(\AA^{2} \times 10^{3}\right)$ for 22. The anisotropic displacement factor exponent takes the form: $-2 \pi^{2}\left[h^{2} a^{* 2} U^{11}+\ldots+2 h k a^{*} b^{*} U^{12}\right]$

\begin{tabular}{|c|c|c|c|c|c|c|}
\hline & $\mathrm{U}^{11}$ & $\mathrm{U}^{22}$ & $\mathrm{U}^{33}$ & $\mathrm{U}^{23}$ & $\mathrm{U}^{13}$ & $\mathrm{U}^{12}$ \\
\hline $\operatorname{Br}(1)$ & $65(1)$ & $33(1)$ & $54(1)$ & $11(1)$ & $-5(1)$ & 11(1) \\
\hline $\mathrm{O}(1)$ & $38(1)$ & $39(1)$ & $36(1)$ & $3(1)$ & $13(1)$ & 1(1) \\
\hline $\mathrm{O}(2)$ & $37(1)$ & $42(1)$ & $41(1)$ & $9(1)$ & $7(1)$ & $-9(1)$ \\
\hline $\mathrm{O}(3)$ & $71(2)$ & $117(2)$ & $50(1)$ & $-45(1)$ & $27(1)$ & $-44(2)$ \\
\hline $\mathrm{O}(4)$ & $28(1)$ & $86(2)$ & $59(1)$ & $24(1)$ & $3(1)$ & $-9(1)$ \\
\hline $\mathrm{O}(5)$ & $56(1)$ & $51(1)$ & $93(2)$ & $30(1)$ & $-10(1)$ & $3(1)$ \\
\hline $\mathrm{N}(1)$ & $28(1)$ & $32(1)$ & $45(1)$ & $-2(1)$ & $9(1)$ & $4(1)$ \\
\hline $\mathrm{C}(1)$ & $26(1)$ & $32(1)$ & $32(1)$ & $5(1)$ & $2(1)$ & $0(1)$ \\
\hline$C(2)$ & $21(1)$ & $27(1)$ & $24(1)$ & $-2(1)$ & $-1(1)$ & $0(1)$ \\
\hline$C(3)$ & $20(1)$ & $29(1)$ & $28(1)$ & $5(1)$ & $-1(1)$ & 1(1) \\
\hline$C(4)$ & 21(1) & $30(1)$ & $28(1)$ & $3(1)$ & $0(1)$ & $-1(1)$ \\
\hline$C(5)$ & $18(1)$ & $29(1)$ & $31(1)$ & $5(1)$ & $-1(1)$ & $2(1)$ \\
\hline$C(6)$ & $22(1)$ & $24(1)$ & $34(1)$ & $2(1)$ & $-1(1)$ & $2(1)$ \\
\hline$C(7)$ & $22(1)$ & $31(1)$ & $29(1)$ & $4(1)$ & $2(1)$ & $8(1)$ \\
\hline $\mathrm{C}(8)$ & $20(1)$ & $33(1)$ & $38(1)$ & 11(1) & $-1(1)$ & $-1(1)$ \\
\hline$C(9)$ & $29(1)$ & $29(1)$ & $41(1)$ & $1(1)$ & $-3(1)$ & $-6(1)$ \\
\hline$C(10)$ & $27(1)$ & $34(1)$ & $32(1)$ & $-2(1)$ & $1(1)$ & $4(1)$ \\
\hline $\mathrm{C}(11)$ & $36(1)$ & $50(2)$ & $41(2)$ & $13(1)$ & $8(1)$ & 1(1) \\
\hline$C(12)$ & $31(1)$ & $55(2)$ & $32(1)$ & $5(1)$ & $2(1)$ & $-10(1)$ \\
\hline$C(13)$ & $22(1)$ & $31(1)$ & $56(2)$ & $13(1)$ & $-1(1)$ & $0(1)$ \\
\hline$C(14)$ & $20(1)$ & $29(1)$ & $23(1)$ & $3(1)$ & $6(1)$ & $-2(1)$ \\
\hline$C(15)$ & $34(1)$ & $32(1)$ & $27(1)$ & $-1(1)$ & $-5(1)$ & $-4(1)$ \\
\hline$C(16)$ & $36(1)$ & $40(1)$ & $29(1)$ & $9(1)$ & $-7(1)$ & $0(1)$ \\
\hline$C(17)$ & $31(1)$ & $24(1)$ & $35(1)$ & $10(1)$ & $4(1)$ & $2(1)$ \\
\hline$C(18)$ & $33(1)$ & $29(1)$ & $31(1)$ & $0(1)$ & $-1(1)$ & $-3(1)$ \\
\hline$C(19)$ & $27(1)$ & $34(1)$ & $26(1)$ & $3(1)$ & $-5(1)$ & $-1(1)$ \\
\hline
\end{tabular}


Table 5. Hydrogen coordinates ( $\left.\times 10^{4}\right)$ and isotropic displacement parameters $\left(\AA^{2} \times 10^{3}\right)$ for 22 .

\begin{tabular}{|c|c|c|c|c|}
\hline & $\mathrm{x}$ & $\mathrm{y}$ & z & $\mathrm{U}(\mathrm{eq})$ \\
\hline $\mathrm{H}(1 \mathrm{~A})$ & 13766 & 8980 & 1477 & 36 \\
\hline $\mathrm{H}(1 \mathrm{~B})$ & 13680 & 7545 & 1332 & 36 \\
\hline $\mathrm{H}(2)$ & 11296 & 7952 & 635 & 29 \\
\hline $\mathrm{H}(4 \mathrm{~A})$ & 8416 & 9015 & 1498 & 32 \\
\hline $\mathrm{H}(4 \mathrm{~B})$ & 8433 & 7907 & 995 & 32 \\
\hline $\mathrm{H}(6)$ & 7240 & 8755 & 2600 & 32 \\
\hline $\mathrm{H}(9)$ & 5331 & 4856 & 1934 & 40 \\
\hline $\mathrm{H}(10)$ & 7241 & 5970 & 1293 & 37 \\
\hline $\mathrm{H}(11 \mathrm{~A})$ & 3920 & 6409 & 3993 & 51 \\
\hline $\mathrm{H}(11 \mathrm{~B})$ & 2702 & 7009 & 3463 & 51 \\
\hline $\mathrm{H}(12 \mathrm{~A})$ & 10361 & 7981 & 2608 & 59 \\
\hline $\mathrm{H}(12 \mathrm{~B})$ & 10752 & 9281 & 2289 & 59 \\
\hline $\mathrm{H}(12 \mathrm{C})$ & 12194 & 8280 & 2348 & 59 \\
\hline $\mathrm{H}(13)$ & 10560 & 6144 & 1172 & 44 \\
\hline $\mathrm{H}(15)$ & 9748 & 9300 & 37 & 37 \\
\hline $\mathrm{H}(16)$ & 8807 & 11265 & -220 & 42 \\
\hline $\mathrm{H}(18)$ & 11282 & 12597 & 1336 & 37 \\
\hline $\mathrm{H}(19)$ & 12176 & 10636 & 1595 & 35 \\
\hline
\end{tabular}


Table 6. Torsion angles $\left[^{\circ}\right]$ for 22.

\begin{tabular}{|c|c|}
\hline $\mathrm{O}(3)-\mathrm{N}(1)-\mathrm{C}(1)-\mathrm{C}(2)$ & $-31.4(3)$ \\
\hline $\mathrm{O}(4)-\mathrm{N}(1)-\mathrm{C}(1)-\mathrm{C}(2)$ & $147.4(2)$ \\
\hline $\mathrm{N}(1)-\mathrm{C}(1)-\mathrm{C}(2)-\mathrm{C}(14)$ & $-68.3(2)$ \\
\hline $\mathrm{N}(1)-\mathrm{C}(1)-\mathrm{C}(2)-\mathrm{C}(3)$ & $163.48(17)$ \\
\hline $\mathrm{C}(14)-\mathrm{C}(2)-\mathrm{C}(3)-\mathrm{C}(13)$ & $163.34(19)$ \\
\hline $\mathrm{C}(1)-\mathrm{C}(2)-\mathrm{C}(3)-\mathrm{C}(13)$ & $-69.6(2)$ \\
\hline $\mathrm{C}(14)-\mathrm{C}(2)-\mathrm{C}(3)-\mathrm{C}(12)$ & $-74.0(2)$ \\
\hline $\mathrm{C}(1)-\mathrm{C}(2)-\mathrm{C}(3)-\mathrm{C}(12)$ & $53.0(2)$ \\
\hline $\mathrm{C}(14)-\mathrm{C}(2)-\mathrm{C}(3)-\mathrm{C}(4)$ & $49.3(2)$ \\
\hline $\mathrm{C}(1)-\mathrm{C}(2)-\mathrm{C}(3)-\mathrm{C}(4)$ & $176.35(18)$ \\
\hline$C(13)-C(3)-C(4)-C(5)$ & $55.2(3)$ \\
\hline $\mathrm{C}(12)-\mathrm{C}(3)-\mathrm{C}(4)-\mathrm{C}(5)$ & $-66.5(2)$ \\
\hline $\mathrm{C}(2)-\mathrm{C}(3)-\mathrm{C}(4)-\mathrm{C}(5)$ & $168.03(18)$ \\
\hline $\mathrm{C}(3)-\mathrm{C}(4)-\mathrm{C}(5)-\mathrm{C}(10)$ & $-91.7(3)$ \\
\hline$C(3)-C(4)-C(5)-C(6)$ & $90.0(2)$ \\
\hline $\mathrm{C}(10)-\mathrm{C}(5)-\mathrm{C}(6)-\mathrm{C}(7)$ & $0.3(3)$ \\
\hline$C(4)-C(5)-C(6)-C(7)$ & $178.59(19)$ \\
\hline $\mathrm{C}(5)-\mathrm{C}(6)-\mathrm{C}(7)-\mathrm{C}(8)$ & $0.6(3)$ \\
\hline $\mathrm{C}(5)-\mathrm{C}(6)-\mathrm{C}(7)-\mathrm{O}(1)$ & $177.5(2)$ \\
\hline $\mathrm{C}(11)-\mathrm{O}(1)-\mathrm{C}(7)-\mathrm{C}(6)$ & $174.3(2)$ \\
\hline $\mathrm{C}(11)-\mathrm{O}(1)-\mathrm{C}(7)-\mathrm{C}(8)$ & $-8.6(2)$ \\
\hline $\mathrm{C}(6)-\mathrm{C}(7)-\mathrm{C}(8)-\mathrm{C}(9)$ & $-0.9(4)$ \\
\hline $\mathrm{O}(1)-\mathrm{C}(7)-\mathrm{C}(8)-\mathrm{C}(9)$ & $-178.3(2)$ \\
\hline $\mathrm{C}(6)-\mathrm{C}(7)-\mathrm{C}(8)-\mathrm{O}(2)$ & $175.7(2)$ \\
\hline $\mathrm{O}(1)-\mathrm{C}(7)-\mathrm{C}(8)-\mathrm{O}(2)$ & $-1.7(2)$ \\
\hline $\mathrm{C}(11)-\mathrm{O}(2)-\mathrm{C}(8)-\mathrm{C}(9)$ & $-172.4(2)$ \\
\hline $\mathrm{C}(11)-\mathrm{O}(2)-\mathrm{C}(8)-\mathrm{C}(7)$ & $11.3(2)$ \\
\hline$C(7)-C(8)-C(9)-C(10)$ & $0.3(3)$ \\
\hline $\mathrm{O}(2)-\mathrm{C}(8)-\mathrm{C}(9)-\mathrm{C}(10)$ & $-175.6(2)$ \\
\hline $\mathrm{C}(6)-\mathrm{C}(5)-\mathrm{C}(10)-\mathrm{C}(9)$ & $-0.9(3)$ \\
\hline $\mathrm{C}(4)-\mathrm{C}(5)-\mathrm{C}(10)-\mathrm{C}(9)$ & $-179.2(2)$ \\
\hline$C(8)-C(9)-C(10)-C(5)$ & $0.6(3)$ \\
\hline $\mathrm{C}(8)-\mathrm{O}(2)-\mathrm{C}(11)-\mathrm{O}(1)$ & $-16.5(2)$ \\
\hline $\mathrm{C}(7)-\mathrm{O}(1)-\mathrm{C}(11)-\mathrm{O}(2)$ & $15.4(2)$ \\
\hline
\end{tabular}




\begin{tabular}{lc}
$\mathrm{C}(12)-\mathrm{C}(3)-\mathrm{C}(13)-\mathrm{O}(5)$ & $-0.7(3)$ \\
$\mathrm{C}(4)-\mathrm{C}(3)-\mathrm{C}(13)-\mathrm{O}(5)$ & $-122.5(3)$ \\
$\mathrm{C}(2)-\mathrm{C}(3)-\mathrm{C}(13)-\mathrm{O}(5)$ & $124.1(3)$ \\
$\mathrm{C}(1)-\mathrm{C}(2)-\mathrm{C}(14)-\mathrm{C}(19)$ & $-51.0(3)$ \\
$\mathrm{C}(3)-\mathrm{C}(2)-\mathrm{C}(14)-\mathrm{C}(19)$ & $75.2(3)$ \\
$\mathrm{C}(1)-\mathrm{C}(2)-\mathrm{C}(14)-\mathrm{C}(15)$ & $130.6(2)$ \\
$\mathrm{C}(3)-\mathrm{C}(2)-\mathrm{C}(14)-\mathrm{C}(15)$ & $-103.2(2)$ \\
$\mathrm{C}(19)-\mathrm{C}(14)-\mathrm{C}(15)-\mathrm{C}(16)$ & $-1.7(3)$ \\
$\mathrm{C}(2)-\mathrm{C}(14)-\mathrm{C}(15)-\mathrm{C}(16)$ & $176.8(2)$ \\
$\mathrm{C}(14)-\mathrm{C}(15)-\mathrm{C}(16)-\mathrm{C}(17)$ & $0.7(4)$ \\
$\mathrm{C}(15)-\mathrm{C}(16)-\mathrm{C}(17)-\mathrm{C}(18)$ & $1.0(4)$ \\
$\mathrm{C}(15)-\mathrm{C}(16)-\mathrm{C}(17)-\mathrm{Br}(1)$ & $-178.14(18)$ \\
$\mathrm{C}(16)-\mathrm{C}(17)-\mathrm{C}(18)-\mathrm{C}(19)$ & $-1.5(4)$ \\
$\mathrm{Br}(1)-\mathrm{C}(17)-\mathrm{C}(18)-\mathrm{C}(19)$ & $177.65(18)$ \\
$\mathrm{C}(17)-\mathrm{C}(18)-\mathrm{C}(19)-\mathrm{C}(14)$ & $0.4(4)$ \\
$\mathrm{C}(15)-\mathrm{C}(14)-\mathrm{C}(19)-\mathrm{C}(18)$ & $1.2(3)$ \\
$\mathrm{C}(2)-\mathrm{C}(14)-\mathrm{C}(19)-\mathrm{C}(18)$ & $-177.3(2)$ \\
& \\
\hline
\end{tabular}

Symmetry transformations used to generate equivalent atoms: 